University of Rhode Island

DigitalCommons@URI

Open Access Dissertations

2017

\title{
Cardiovascular Adverse Events in Patients Receiveing QT Interval Prolonging Medications
}

Yizhou Ye

University of Rhode Island, james0602@gmail.com

Follow this and additional works at: https://digitalcommons.uri.edu/oa_diss

\section{Recommended Citation}

Ye, Yizhou, "Cardiovascular Adverse Events in Patients Receiveing QT Interval Prolonging Medications" (2017). Open Access Dissertations. Paper 589.

https://digitalcommons.uri.edu/oa_diss/589

This Dissertation is brought to you for free and open access by DigitalCommons@URI. It has been accepted for inclusion in Open Access Dissertations by an authorized administrator of DigitalCommons@URI. For more information, please contact digitalcommons-group@uri.edu. 
CARDIOVASCULAR ADVERSE EVENTS IN PATIENTS RECEIVING QT INTERVAL PROLONGING MEDICATIONS

BY

YIZHOU YE

A DISSERTATION SUBMITTED IN PARTIAL FULFILLMENT OF THE

REQUIREMENTS FOR THE DEGREE OF

DOCTOR OF PHILOSOPHY

IN

PHARMACEUTICAL SCIENCES

UNIVERSITY OF RHODE ISLAND 


\section{DOCTOR OF PHILOSOPHY DISSERTATION}

OF

YIZHOU YE

\section{APPROVED:}

Dissertation Committee:

Co-Major Professor Aisling R. Caffrey

Co-Major Professor E Paul Larrat

Kerry L. LaPlante

Marc Hutchison

Nasser H. Zawia

DEAN OF THE GRADUATE SCHOOL

UNIVERSITY OF RHODE ISLAND 


\begin{abstract}
Background

Drug-induced QT interval prolongation is associated with rare but lifethreatening ventricular arrhythmia and sudden death. To study ventricular arrhythmia and sudden cardiac death associated with QT prolongation in large databases, an accurate operational definition of this outcome is needed. Frequently prescribed macrolide and fluoroquinolone antibiotics are associated with torsades de pointes arrhythmia and sudden death. Due to the dose-dependent nature of the risk, concomitant use of multiple QT-prolonging drugs may pose a greater threat than singular use. Several observational epidemiologic studies suggest that azithromycin may have an increased risk of ventricular arrhythmia and sudden cardiac death. Meanwhile, other observational studies observed a relatively similar cardiac toxicity profile with azithromycin, as compared with no antibiotic use or other antibiotics.
\end{abstract}

\title{
Objectives
}

The purpose of the first manuscript in this dissertation is to identify operational definitions of arrhythmias and sudden death associated with QT prolongation used in retrospective database studies and to compare validation results between algorithms. The second manuscript focuses on quantifying the risk of cardiac adverse events related to concurrent concomitant use of QT-prolonging antibiotics and other drugs with similar pro-arrhythmic potential, and the risk factors associated with such events in a national, privately insured population in the United States. The third manuscript 
seeks to assess the risk of ventricular arrhythmia and sudden death for macrolide and fluoroquinolone antibiotics in a national, commercially insured population in the United States and to compare these results with previously published observational studies.

Methods

In the first manuscript, we conducted a systematic literature review using PubMed to identify retrospective studies published between January 1, 2000 and August 31, 2016. We identified and reviewed studies of ventricular arrhythmia or sudden death associated with QT prolongation in large administrative databases. Validation methods and results were also extracted where validation was conducted.

In the second manuscript, we performed a retrospective case-control study using an administrative health claims database from a large national insurer from 2011 to 2013. Cases of ventricular arrhythmia or sudden death due to QT prolongation were selected using a validated algorithm identified from the first manuscript. Four controls were matched to each case on age, sex, and region. Concomitant drug use was defined as overlapping durations of prescriptions of a QT-prolonging antibiotic and one other QT drug of interest. Odds ratios of risk factors and concomitant QTprolonging medication use were calculated using conditional logistic regression.

In the third manuscript, a retrospective cohort study was conducted in the same administrative health claims database from a large national insurer from 2011 to 2013. The study cohort consisted of patients who filled an outpatient prescription for 
macrolide and fluoroquinolone antibiotics, or amoxicillin. Amoxicillin episodes were 1-to-1 matched to macrolide and fluoroquinolone episodes on propensity scores. Inpatient admissions or emergency department visits with a primary diagnosis of ventricular arrhythmia were assessed within 10 days and 30 days of the prescription dispensing. Cox proportional hazard models were used to estimate the hazard ratio.

Findings

In the first manuscript, several algorithms for identifying QT prolongation in large databases have been developed and validated. We found a common algorithm for QT prolongation that was validated in Medicaid, Medicare, and the Italian National Health Service data. We also found a validated operational definition for sudden death in Medicaid data.

In the second manuscript, we found that concomitant and proximal use of QTprolonging antibiotics with other QT medications predicted ventricular arrhythmia or sudden death.

In the third manuscript, azithromycin use and fluoroquinolone use was not associated with an increased cardiac risk compared with amoxicillin. Macrolide antibiotics, as a class, increased the risk of ventricular arrhythmia and sudden death in the 10 days following the prescription dispensing. 


\section{ACKNOWLEDGEMENTS}

Foremost, I would like to express my sincere gratitude to my major advisors Dr. Aisling R. Caffrey and Dean. E P. Larrat. Dean Larrat has been incredibly supportive for the entire time of my study at the University of Rhode Island. He was the first person I spoke to on my first day in Kingston, RI. Dr. Caffrey has been supporting me and challenging me through this entire dissertation project with her knowledge, dedication, and patience. Every conversation I had with her was extremely valuable to my dissertation study as well as personal growth. It would not been possible for me to complete this project without her guidance, inspiration and encouragement. I would also like to thank other members of my dissertation committee, Dr. Kerry LaPlante and Dr. Marc Hutchison for their comments and inputs on the final work.

In addition, I would also like to thank my friends Dr. Jinghua He, Bingcao Wu, Chao Chen, Dr. Yi-Tzai Chen, Robert McConeghy, Ajinkya Pawar, Yuanjun Shen, and Drs. Zhengxi Wei and Jing Yang for their friendship and support all these years. I greatly enjoyed your company and I learned immensely from each one of you.

Lastly, I would like to thank my family for their unconditional love and unlimited support. My parents Kelin Ye and Zheng Jiang have worked hard for decades made tremendous sacrifices so that I could have a chance to pursue my own dreams. More importantly, they taught me to be a better man every single day with their moral values and integrity. I could never thank you enough for what have done for me. 


\section{PREFACE}

Drug-induced QT interval prolongation is associated with rare but life-threatening ventricular arrhythmia and sudden death. Macrolide and fluoroquinolone antibiotics are considered to have a risk of QT prolongation despite conflicting results observed in retrospective studies. Meanwhile, the risk of concomitant use of multiple QTprolonging medications remains largely unknown. The purpose of this dissertation is to quantify the risk of ventricular arrhythmia and sudden death associated with macrolide and fluoroquinolone antibiotics, when exposed alone and in combination with other risk factors.

Following the manuscript format, this dissertation consists of three manuscripts:

Manuscript I. Algorithms used to identify arrhythmias and sudden cardiac death associated with QT prolongation in retrospective studies: a systematic literature review

Manuscript II. Concomitant use of QT-prolonging medications and the risk of ventricular arrhythmia or sudden death

Manuscript III. Risk of drug-induced ventricular arrhythmia and sudden death with QT-prolonging antibiotics 


\section{TABLE OF CONTENTS}

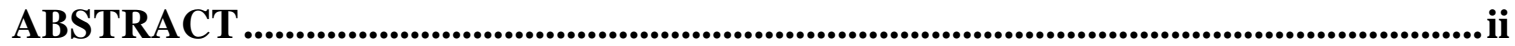

ACKNOWLEDGEMENTS................................................................................

PREFACE ............................................................................................................... vi

TABLE OF CONTENTS.............................................................................................. vii

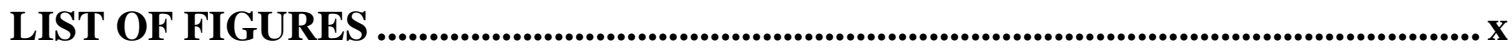

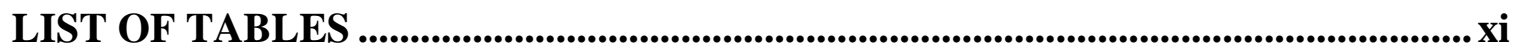

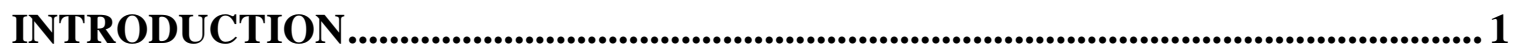

DRUG-INDUCED QT PROLONGATION ............................................................ 1

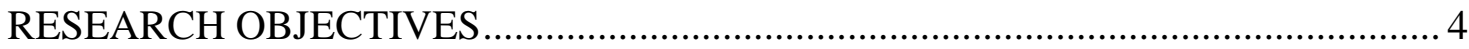

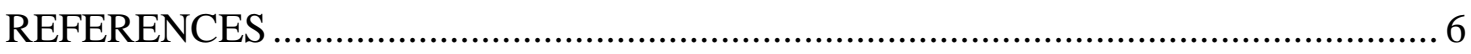

Manuscript I. Algorithms used to identify arrhythmias and sudden cardiac death associated with QT prolongation in retrospective studies: a systematic literature review

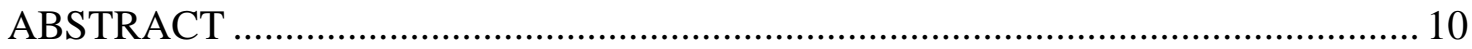

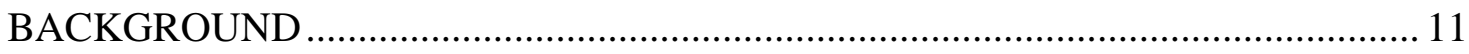

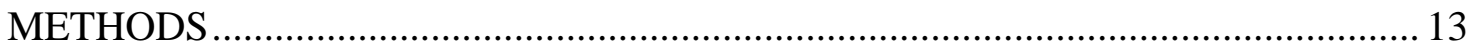

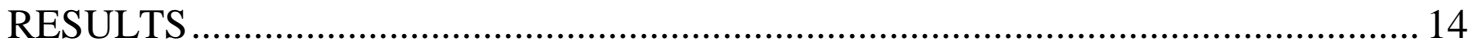

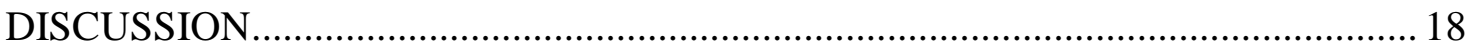

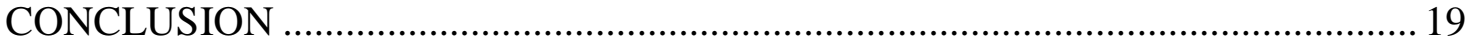


Manuscript II. Concomitant use of QT-prolonging medications and the risk of ventricular arrhythmia and sudden death.

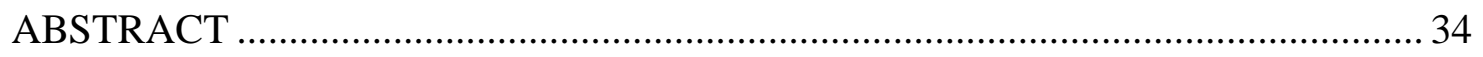

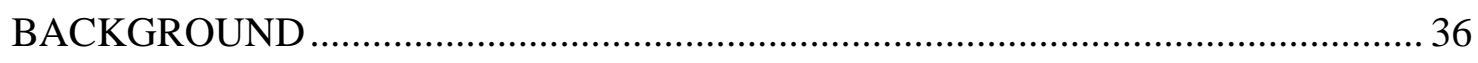

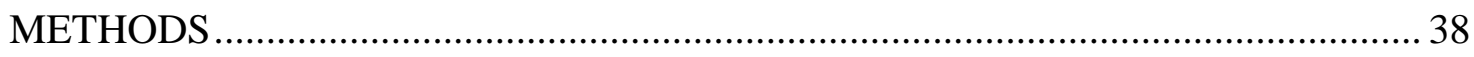

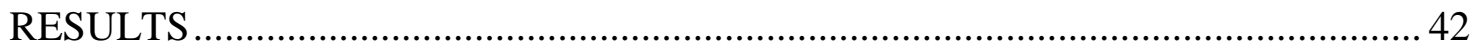

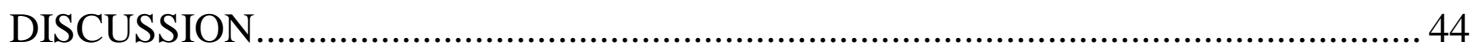

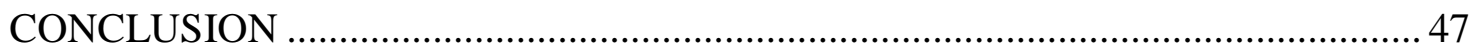

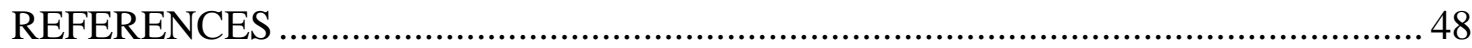

Manuscript III. Risk of drug-induced ventricular arrhythmia and sudden death with QT-prolonging antibiotics.........................................................................................56

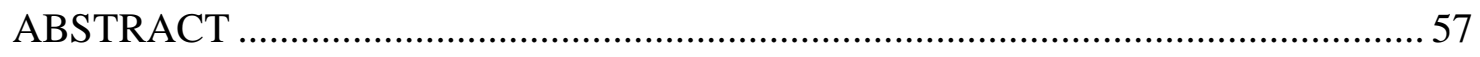

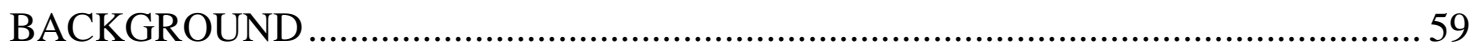

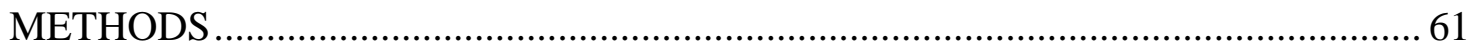

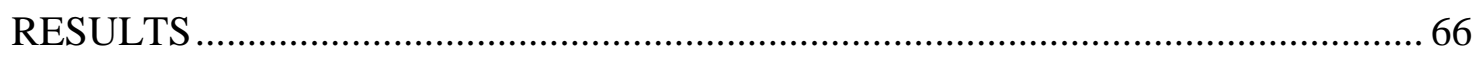

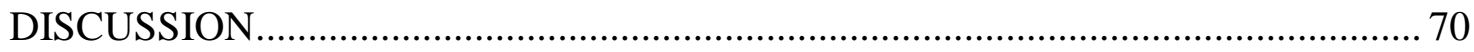

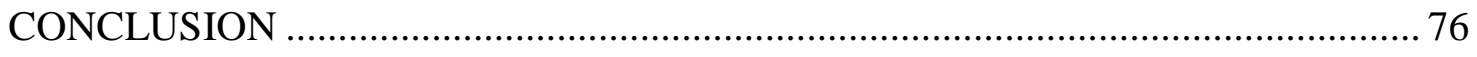

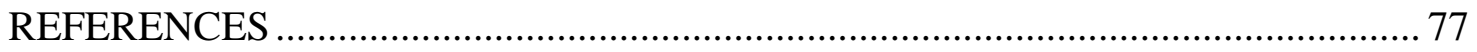

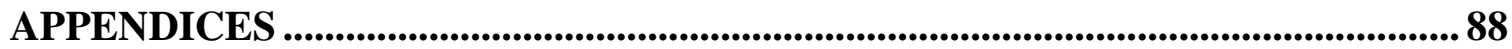


Appendix A. Risk factors for ventricular arrhythmia or sudden death

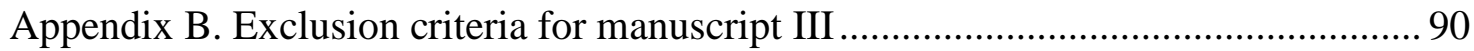

Appendix C. List of covariates for propensity score model .................................... 92

Appendix D. Distributions of propensity scores in matched study cohorts ................. 94

Appendix E. Demographics and clinical characteristics of the matched cohorts.......... 95

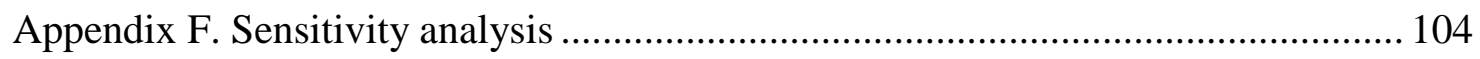

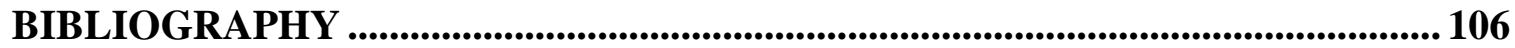




\section{LIST OF FIGURES}

Figure

Figure 1.1 Flow chart of literature search and selection process

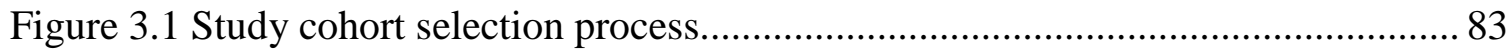

Figure 3.2 Cumulative incidence of ventricular arrhythmia and sudden death with azithromycin and amoxicillin .

Figure 3.3 Cumulative incidence of ventricular arrhythmia and sudden death with macrolides and amoxicillin

Figure 3.4 Cumulative incidence of ventricular arrhythmia and sudden death with

fluoroquinolones and amoxicillin 86

Figure 3.5 Cumulative incidences of ventricular arrhythmia and sudden death with fluoroquinolones and amoxicillin in all diagnoses 


\section{LIST OF TABLES}

Table

Page

Table 1.1 Literature search strategy and number of searched studies. 23

Table 1.2 Characteristics of Reviewed retrospective large database studies 25

Table 1.3 The most common ICD-9 diagnosis codes for ventricular arrhythmias related to QT prolongation 30

Table 1.4 The most common ICD-9 codes for sudden death related to QT prolongation 31

Table 2.1 Demographics and clinical characteristics of cases and controls 51

Table 2.2 Concomitant QT drug use and predictors of ventricular arrhythmia or sudden death 53

Table 2.3 Proximal QT drug use ( \pm 15 days) and predictors of ventricular arrhythmia or sudden death

Table 2.4 Number of QT-prolonging medications and predictors of ventricular arrhythmia or sudden death 55

Table 3.1 Demographics and clinical characteristics of the study population 81 


\section{INTRODUCTION}

\section{DRUG-INDUCED QT PROLONGATION}

Drug-induced QT interval prolongation is associated with rare but lifethreatening ventricular arrhythmia and sudden death. Retrospective pharmacoepidemiologic studies are often conducted to assess risks of adverse events of medications. ${ }^{1,2}$ Based on such studies, QT prolongation has informed black box warnings and drug withdrawals. ${ }^{3}$ In 2013, azithromycin received a black box warning for potential risk of QT prolongation and fatal cardiac arrhythmias based on the findings of a large retrospective pharmacoepidemiologic study. ${ }^{4,5}$ In this era of big data, particularly for studies of rare outcomes, such as acute ventricular arrhythmia and sudden cardiac death associated with QT prolongation, an accurate operational definition of the outcome is needed. Physician review of an electrocardiogram is the gold standard for identifying QT prolongation, however it has limited use in retrospective database studies. ${ }^{6}$ First, it is often not available in large claims-based databases. Secondly, studies which rely on medical charts are often under-powered to study a rare outcome within a small study population. ${ }^{7}$ Lastly, electrocardiogram results are not able to capture arrhythmia and sudden death which occurred outside of the hospital setting.

Macrolide and fluoroquinolone antibiotics are among the most frequently prescribed drugs that are associated QT prolongation, which can lead to torsades de

pointes arrhythmia and sudden death. ${ }^{4,5,8-11}$ As dose-dependent risks have been identified from pharmacokinetic and pharmacodynamic research with QT-prolonging 
medications, greater risk may exist with concomitant use of multiple QT-prolonging drugs. ${ }^{12,13}$ Torsades de pointes is also associated with other risk factors such as older age, female sex, hypokalemia, history of heart diseases, and renal impairment. ${ }^{14,15}$ Numerous QT-prolonging drugs are metabolized by the same group of cytochrome isoenzymes (mostly CYP3A4). ${ }^{13,16}$ When exposed together, one drug will serve as the other's metabolic enzyme inhibitors and cause drug accumulation which further increases the risk of drug-induced QT prolongation, a concentration-related event. In addition, another mechanism for increased risk would be the medications serving as each other's synergist or antagonist. ${ }^{13,17}$ Some macrolides and fluoroquinolones are also known to have pharmacokinetic drug-drug interactions. ${ }^{18}$ Clarithromycin, erythromycin, and ciprofloxacin are all strong inhibitors of cytochrome P450 isoenzymes. ${ }^{13}$

All macrolide antibiotics are considered to have QT-prolonging potential. Azithromycin is not a CYP3A4 inhibitor, and was therefore considered to be safer than the rest of its class. However, several recent observational epidemiologic studies suggest that azithromycin may have an increased risk of ventricular arrhythmia and sudden cardiac death afterall. ${ }^{4,19,20}$ One retrospective cohort study published in 2012 reported a higher risk of cardiovascular death associated with azithromycin compared with no antibiotic use in a Medicaid population. ${ }^{4}$ One year later, the United States Food and Drug Administration (FDA) issued a warning to urge healthcare professionals to consider potential cardiovascular risks when prescribing azithromycin. ${ }^{5}$ Subsequent observational studies among Veterans Affairs patients and 
in the general population of Taiwan reported similar increased risks of serious arrhythmia, cardiovascular death, and all-cause mortality with azithromycin. ${ }^{19,20}$

Alternatively, several observational studies reported a safer cardiac toxicity profile with azithromycin, as compared with no antibiotic use or other antibiotics ${ }^{21-23}$ In the Danish adult population, azithromycin use was not associated with an increased risk of cardiovascular death compared to either no antibiotic use or penicillin- $\mathrm{V} .^{22}$ Another study among the general population of Ontario, Canada found that macrolide antibiotic use was not associated with a higher risk of ventricular arrhythmia compared with non-macrolide antibiotics. ${ }^{21}$ 


\section{RESEARCH OBJECTIVES}

A number of pharmacoepidemiologic drug safety studies have sought to quantify the risk of drug-induced ventricular arrhythmia and sudden death. The algorithms identifying ventricular arrhythmia and sudden cardiac death associated with QT prolongation have varied between studies. ${ }^{19,20,24-26}$ Identifying a validated algorithm is needed in order to have an accurate definition of this outcome and for comparability between studies. Interactions among QT-prolonging agents are wellstudied in the field of pharmacokinetics and pharmacodynamics but are less wellstudied in the field of pharmacoepidemiology. Concomitant use of multiple QTprolonging drugs may pose a greater threat than singular use. Conflicting cardiac safety profiles for QT-prolonging antibiotics, including azithromycin, have been observed in different populations worldwide. These populations varied in age, social economic status, and disease burden.

Based on these unanswered questions, this dissertation has three objectives related to drug-induced QT interval prolongation, namely:

1. To identify the operational definitions of arrhythmias and sudden death associated with QT prolongation used in retrospective database studies and compare validation results between algorithms.

2. To quantify the risk of cardiac adverse events related to concomitant use of QT-prolonging antibiotics and other drugs with similar pro-arrhythmic potential, and other risk factors associated with such events in a national, privately-insured population in the United States. 
3. To assess the risk of ventricular arrhythmia and sudden death associated with macrolide and fluoroquinolone antibiotics in a national, commercially insured population in the United States and compare these results with previously published observational studies. 


\section{REFERENCES}

1. European Medicines Agency. Guideline on good pharmacovigilance practices (GVP) Module VIII - Post-authorisation safety studies (Rev 2) 2016;

http://www.ema.europa.eu/docs/en_GB/document_library/Scientific_guideline/2012/0 6/WC500129137.pdf. Accessed September 20, 2016.

2. U.S. Department of Health and Human Services USFDA. Good Pharmacovigilance Practices and Pharmacoepidemiologic Assessment. 2005; http://www.fda.gov/downloads/regulatoryinformation/guidances/ucm126834.pdf. Accessed September 20, 2016.

3. Chiang CE. Congenital and acquired long QT syndrome. Current concepts and management. Cardiology in review. 2004;12(4):222-234.

4. Ray WA, Murray KT, Hall K, Arbogast PG, Stein CM. Azithromycin and the risk of cardiovascular death. N Engl J Med. 2012;366(20):1881-1890.

5. U.S. Department of Health and Human Services USFDA. FDA Drug Safety Communication: Azithromycin (Zithromax or Zmax) and the risk of potentially fatal heart rhythms. 2013; http://www.fda.gov/Drugs/DrugSafety/ucm341822.htm. Accessed September 20, 2016.

6. Denny JC, Miller RA, Waitman LR, Arrieta MA, Peterson JF. Identifying QT prolongation from ECG impressions using a general-purpose Natural Language Processor. Int J Med Inform. 2009;78 Suppl 1:S34-42.

7. Strom BL KS. Textbook of Pharmacoepidemiology. 1st ed.

8. Briasoulis A, Agarwal V, Pierce WJ. QT prolongation and torsade de pointes induced by fluoroquinolones: infrequent side effects from commonly used medications. Cardiology. 2011;120(2):103-110.

9. Owens RC, Jr., Nolin TD. Antimicrobial-associated QT interval prolongation: pointes of interest. Clinical infectious diseases : an official publication of the Infectious Diseases Society of America. 2006;43(12):1603-1611.

10. Liu HH. Safety profile of the fluoroquinolones: focus on levofloxacin. Drug Saf. 2010;33(5):353-369.

11. Mehrzad R, Barza M. Weighing the adverse cardiac effects of fluoroquinolones: A risk perspective. J Clin Pharmacol. 2015;55(11):1198-1206.

12. Hoffler D, Koeppe P, Paeske B. Pharmacokinetics of azithromycin in normal and impaired renal function. Infection. 1995;23(6):356-361. 
13. Dresser GK, Spence JD, Bailey DG. Pharmacokinetic-pharmacodynamic consequences and clinical relevance of cytochrome P450 3A4 inhibition. Clin Pharmacokinet. 2000;38(1):41-57.

14. Roden DM. Drug-induced prolongation of the QT interval. N Engl J Med. 2004;350(10):1013-1022.

15. Yap YG, Camm AJ. Drug induced QT prolongation and torsades de pointes. Heart. 2003;89(11):1363-1372.

16. Chen HL, Hsiao FY. Domperidone, cytochrome P450 3A4 isoenzyme inhibitors and ventricular arrhythmia: a nationwide case-crossover study. Pharmacoepidemiology and drug safety. 2015;24(8):841-848.

17. Aerssens J, Paulussen AD. Pharmacogenomics and acquired long QT syndrome. Pharmacogenomics. 2005;6(3):259-270.

18. van Noord C, Eijgelsheim M, Stricker BH. Drug- and non-drug-associated QT interval prolongation. British journal of clinical pharmacology. 2010;70(1):16-23.

19. Rao GA, Mann JR, Shoaibi A, et al. Azithromycin and levofloxacin use and increased risk of cardiac arrhythmia and death. Annals of family medicine. 2014;12(2):121-127.

20. Chou HW, Wang JL, Chang CH, Lai CL, Lai MS, Chan KA. Risks of cardiac arrhythmia and mortality among patients using new-generation macrolides, fluoroquinolones, and beta-lactam/beta-lactamase inhibitors: a Taiwanese nationwide study. Clinical infectious diseases : an official publication of the Infectious Diseases Society of America. 2015;60(4):566-577.

21. Trac MH, McArthur E, Jandoc R, et al. Macrolide antibiotics and the risk of ventricular arrhythmia in older adults. CMAJ. 2016;188(7):E120-129.

22. Svanstrom H, Pasternak B, Hviid A. Use of azithromycin and death from cardiovascular causes. N Engl J Med. 2013;368(18):1704-1712.

23. Mortensen EM, Halm EA, Pugh MJ, et al. Association of azithromycin with mortality and cardiovascular events among older patients hospitalized with pneumonia. JAMA. 2014;311(21):2199-2208.

24. Chung CP, Murray KT, Stein CM, Hall K, Ray WA. A computer case definition for sudden cardiac death. Pharmacoepidemiology and drug safety. 2010;19(6):563-572.

25. Hennessy S, Leonard CE, Freeman CP, et al. Validation of diagnostic codes for outpatient-originating sudden cardiac death and ventricular arrhythmia in Medicaid and Medicare claims data. Pharmacoepidemiology and drug safety. 2010;19(6):555562 . 
26. Hanrahan JP, Choo PW, Carlson W, Greineder D, Faich GA, Platt R.

Terfenadine-associated ventricular arrhythmias and QTc interval prolongation. A retrospective cohort comparison with other antihistamines among members of a health maintenance organization. Annals of epidemiology. 1995;5(3):201-209. 
Manuscript I. Algorithms used to identify arrhythmias and sudden cardiac death associated with QT prolongation in retrospective studies: a systematic literature review

Yizhou Ye, M.S. ${ }^{1}$, E P. Larrat, Ph.D. ${ }^{1}$, and Aisling R. Caffrey, Ph.D., M.S. ${ }^{1,2,3}$

1. College of Pharmacy, Department of Pharmacy Practice, University of Rhode Island, 7 Greenhouse Rd, Kingston, RI, United States

2. Veterans Affairs Medical Center, Infectious Diseases Research Program and Center of Innovation in Long Term Services and Supports, Providence, RI, United States

3. School of Public Health, Brown University, Providence, RI, United States

Corresponding Author: Aisling R. Caffrey, PhD, MS, Assistant Professor, University of Rhode Island; 7 Greenhouse Road, Kingston, RI 02881; office: (401) 874-5320; e-mail: Aisling_Caffrey@uri.edu

Abstract word count: 178

Manuscript word count: 1723

Table/figure count: $4 / 1$

Funding: Unfunded.

Keywords: Azithromycin, macrolide, fluoroquinolone, drug safety, QT prolongation, ventricular arrhythmia, retrospective study

Target journal: INQUIRY

Publication status: Submitted for review 


\section{ABSTRACT}

Drug-induced QT prolongation can lead to ventricular arrhythmia and sudden cardiac death. As ventricular arrhythmia and sudden cardiac death are rare events, large administrative databases can be used to inform pharmacoepidemiologic drug safety studies. In order to compare event rates between studies, validated operational definitions of these events are needed. We conducted a systematic literature review in PubMed to identify diagnosis code algorithms for arrhythmias and sudden cardiac death. Twenty-two studies were included in the review. For arrhythmias, a common operational definition was identified in $23 \%$ of the reviewed studies $(n=5)$, with an average positive predictive value (PPV) of 85\% from hospital stays and emergency visits. For sudden cardiac death, codes for cause of death was used in $14 \%$ of studies (n=3), with an average PPV of 88\%. Further validation of a common International Classification of Diseases, 10th Edition (ICD-10) algorithm is needed for each event. In conclusion, researchers should utilize a common, validated algorithm, such as the one identified in our review to operationally define these events to ensure comparability between new research and the existing literature. 


\section{BACKGROUND}

Unexpected sudden death is rare, with an estimated incidence ranging from 50 to 100 per 100,000 individuals per year in Europe and North America. ${ }^{1,2}$ Acute ventricular arrhythmia may account for over $80 \%$ of sudden cardiac deaths. ${ }^{3}$ Retrospective pharmacoepidemiologic studies are used to assess risks attributed to drug exposures. ${ }^{4,5}$ Based on such studies, QT prolongation has informed black box warnings and drug withdrawals. ${ }^{6}$ Terfenadine and cisapride were withdrawn in the United States (US) in 1998 and 2000 respectively, due to their association with QT prolongation and ventricular arrhythmia. ${ }^{7,8}$ More recently, in 2013, azithromycin received a black box warning for potential risk of QT prolongation and fatal cardiac arrhythmias based on the findings of a large retrospective pharmacoepidemiologic study. 9,10

In this era of big data, particularly for studies of rare outcomes, such as acute ventricular arrhythmia and sudden death associated with QT prolongation, an accurate operational definition of the outcome is needed. Physician review of an electrocardiogram (ECG) is the gold standard for identifying QT prolongation, however it has limited use in retrospective database studies. ${ }^{11}$ First, ECG results are often not available in large databases. Further, a study identifying events by manual medical record review of ECGs and physician notes would likely be under-powered to quantify the exposure-outcome relationship. Lastly, ECG results for acute ventricular arrhythmia and sudden death which occurred outside of the hospital will not be available, and would result in underestimation of these events. 
A number of retrospective pharmacoepidemiologic drug safety studies have sought to quantify the association between these rare outcomes and different medication exposures, however the algorithms identifying ventricular arrhythmia and sudden death associated with QT prolongation have varied between studies. ${ }^{12-16}$ Further the performance of these varying algorithms has not been compared. The objective of this systematic literature review was to identify the operational definitions of arrhythmias and sudden death associated with QT prolongation used in retrospective database studies and compare validation results between algorithms. 


\section{METHODS}

A systematic literature review in the PubMed electronic databases was conducted to identify retrospective studies from peer-reviewed journals. A predetermined search strategy developed by Mini-Sentinel researchers (Table 1.1) was used to select qualifying studies published between January 1, 2000 and August 31, 2016. ${ }^{17}$ This literature review was limited to studies in humans and published in English. Other inclusion criteria were: retrospective studies in large administrative databases; studies that identified ventricular arrhythmia and sudden death associated with QT prolongation; studies that only defined QT prolongation with ECG. Titles and abstracts of studies identified by the search were screened against the inclusion criteria. Qualifying or uncertain studies from title and abstract review underwent further full-text review for selection. References of selected studies were also examined for inclusion. Data extracted included operational definitions and administrative codes used for identifying ventricular arrhythmia and sudden death associated with QT prolongation. Other aspects of study design including publication

year, study setting and population, and sample size, were also collected. Validation methods and results were also extracted where validation was conducted. 


\section{RESULTS}

The search strategy identified 1,237 studies. After title and abstract review, 57 studies were selected for full-text review, after which, 22 (38.6\%) studies were selected for inclusion. The literature search, review, and selection process was demonstrated in Figure 1.1 with results of each step in Table 1.1.

The characteristic of included studies is showed in Table 1.2. Half of the included studies were published after 2012, which was the year a previous literature review of validated methods for identifying ventricular arrhythmia was published. ${ }^{17}$ Approximately half of the studies $(n=12,55 \%)$ were conducted in US populations, using Medicare or Medicaid data $(n=9,41 \%),{ }^{8,9,12,13,18-22}$ commercial health plan data $(n=2,9 \%),{ }^{7,16}$ or Veterans Health Administration data $(n=1,4.5 \%){ }^{15}$ The remaining studies $(n=10,45 \%)$ were conducted in: European databases $(n=5,23 \%)$ from the Netherlands, ${ }^{23}$ France, ${ }^{24}$ Italy, ${ }^{25}$ Denmark, ${ }^{26,27}$ and Sweden $^{27}$; the Taiwanese National Health Insurance Research Database $(n=3,14 \%)^{14,28,29}$; and Canadian provincial databases $(n=2,9 \%){ }^{30,31}$ International Classification of Diseases, Ninth Revision (ICD-9) codes were used to identify cardiac arrhythmias and/or sudden cardiac deaths in 17 studies (77\%), ${ }^{7-9,12,13,15,16,18-23,25,28,29,31}$ while 4 studies (18\%) used International Classification of Diseases, Tenth Revision (ICD-10) codes, ${ }^{24,26,27,30}$ and 1 study (4.5\%) used both ICD-9 and ICD-10 codes. All studies used diagnosis codes for event definitions and no procedure codes were used. ${ }^{14}$

ICD-9 diagnosis codes for arrhythmias were used in 15 out of 22 studies

(68\%). Shared by 5 studies, 8,13,19,20,25 the most common algorithm (Table 1.3) used 
hospital stays and ED visits for ventricular arrhythmia to identify QT prolongation. Validation was conducted in three of these studies by review of the medical record. When limited to principle diagnosis, this algorithm had a positive predictive value (PPV) of $80 \%$ and $94 \%$ for hospitalization diagnosis and ED diagnosis respectively in Medicaid and Medicare data and the overall PPV was $85 \% .{ }^{13}$ Other validation studies in a Medicaid and Medicare combined population and in an Italian population showed a PPV of $85 \%$ and $73 \%$, respectively. ${ }^{13,25}$ One variation of the algorithm (excluded ICD-9 code 798) was used by one of the Taiwanese studies and a Medicaid/HealthCore study but neither study evaluated the validation of the algorithm. ${ }^{22,28}$ Another variation (only included ICD-9 codes 427.1, 427.4, and 427.5) of this algorithm in a study using Medicare data demonstrated a PPV of 93\%, as validated by medical record review. ${ }^{18}$

The next most common algorithm for arrhythmias was utilized in two studies, one from the Netherlands and the other from Canada. ${ }^{23,31}$ The algorithm identified QT prolongation from hospital stays with primary diagnoses of arrhythmias. Only the Dutch study validated the algorithm, where a review of medical records was used to verify the ICD-9 codes used for ventricular arrhythmia and cardiac arrest (PPV 82\%) and ICD-9 codes for unspecified cardiac arrhythmias (PPV 10\%). ${ }^{23}$ The five other studies each used a different algorithm to identify potential QT prolongation. The Harvard community health plan study used select ICD-9 subcodes under 426, 427, 429, 780, and 785 from hospital stays and ED visits, with a low PPV of $4 \%{ }^{16}$ The United Healthcare study included office visits and inpatient stays with diagnoses of arrhythmic events (ICD-9 codes 426.x, 427.x) but this approach also had low a PPV of 
$10 \%$ based on a review of medical records. ${ }^{7}$ Three other algorithms were used in two of the Taiwanese studies and the Veterans Health Administration study and none of these studies validated their operational definition. ${ }^{14,15,29}$

ICD-10 diagnosis codes for acute ventricular arrhythmia were used in 3 studies (13.6\%). A French study, ${ }^{24}$ a Denmark-Sweden bi-national study, ${ }^{27}$ and one of the Canadian studies ${ }^{30}$ each used a different algorithm for identifying QT prolongation in hospital stays or ED visits. The Canadian study used ICD-10 codes I47.2 and I49.0 and a manual chart review was conducted in 202 charts resulting in a PPV of $92 \% .^{30}$ The French study used ICD-10 codes I46.1, I47.2, and I49.0 from hospital discharge summaries. In comparison with ECG records, this algorithm had a PPV of $60 \%{ }^{24}$ The bi-national study used ICD-10 codes I47.2, I49.0, I49.3, I46.0, I46.1, I46.9, R96.0, and R96.1 and no validation was conducted. ${ }^{27}$

Besides arrhythmias, sudden death has also been used to identify QT prolongation in large retrospective databases, with both ICD-9 and ICD-10 codes. ICD-9 codes for sudden death were used in 4 of the 22 (18.2\%) reviewed studies. An algorithm used in the Tennessee Medicaid study to identify sudden death associated QT prolongation was also used in 2 other studies (Table 1.4). ${ }^{12}$ This algorithm excluded deaths with terminal institutional stays or terminal procedures inconsistent with unresuscitated cardiac arrest. A series of ICD-9 codes for causes of death were used to define plausible sudden cardiac death. This algorithm was validated by medical record review in a general Medicaid population and in opioid users, and the PPV of this algorithm was $87 \%$ and $88 \%$, respectively. ${ }^{12,21}$ Another algorithm used by 
one of the Taiwanese studies defined the study outcome as unspecific cardiovascular death (ICD-9 codes 401-449) and this algorithm was not validated. ${ }^{14}$

The Danish study and one of the Taiwanese studies identified death from cardiovascular causes using ICD-10 codes ( $\mathrm{n}=2,9.1 \%) .{ }^{14,26}$ Codes used in the Danish study were I00-I99 (diseases of the circulatory system) and R96.x (other sudden death, cause unknown). ${ }^{26}$ Codes used in the Taiwanese study were I10-I79 (hypertensive diseases, ischemic heart diseases, pulmonary heart disease and diseases of pulmonary circulation, other forms of heart disease, and cerebrovascular diseases). ${ }^{14}$ Neither study performed validation of their algorithms. 


\section{DISCUSSION}

We identified common, validated operational definitions for identifying arrhythmias and sudden death associated with QT prolongation in large retrospective database studies. The most common algorithm for ventricular arrhythmia (Table 1.3) and sudden death (Table 1.4) had an average PPV of $85 \%$ and $88 \%$, respectively. These two algorithms were validated in various patient populations, including Medicaid and Medicare, administrative claims databases, and several European and Canadian databases. For ICD-9 arrhythmias algorithms, the PPV was highest when the limited to principle diagnoses and addition less specific subcodes decreases the PPV. ${ }^{7,8}$ Though one ICD-10 algorithm for arrhythmias was found to have a high PPV, this algorithm has not been used or validated in another database. ${ }^{30}$ The sole ICD-9 sudden death algorithms had a high PPV for identifying QT prolongation among patients with a low risk of sudden cardiac death. ${ }^{12}$

Our study has a few limitations. Firstly, the literature search terms might miss some other studies that also identified QT prolongation in retrospective databases. We attempted to address this limitation by examining the references of reviewed studies for additional studies and found no additional eligible studies in the references. Secondly, the true incidence of QT prolongation is unknown as QT prolongations can occur without noticeable symptoms. ${ }^{32,33}$ As such, validation studies could only calculate the PPV but not sensitivity or specificity. However, as a PPV is the proportion of true positive in tested positive, validated algorithms with high PPV may accurately identify cases captured in large retrospective databases. 


\section{CONCLUSION}

A common, validated algorithm for QT prolongation related arrhythmias was identified and validated in Medicaid, Medicare, and the Italian National Health Service data, as was a validated operational definition for sudden death in Medicaid data. Consistency between studies is necessary for establishing causal relationships between medications and rare adverse events, such as prolonged QT associated arrhythmias and sudden cardiac death. As such, to ensure comparability between new research and the existing literature, researchers should utilize a common, validated algorithm, such as the one identified in our review, to operationally define these events. 


\section{REFERENCES}

1. Fishman GI, Chugh SS, Dimarco JP, et al. Sudden death prediction and prevention: report from a National Heart, Lung, and Blood Institute and Heart Rhythm Society Workshop. Circulation. 2010;122(22):2335-2348.

2. Goldberger JJ, Buxton AE, Cain M, et al. Risk stratification for arrhythmic sudden cardiac death: identifying the roadblocks. Circulation. 2011;123(21):24232430 .

3. Josephson M, Wellens HJ. Implantable defibrillators and sudden cardiac death. Circulation. 2004;109(22):2685-2691.

4. European Medicines Agency. Guideline on good pharmacovigilance practices (GVP) Module VIII - Post-authorisation safety studies (Rev 2) 2016; http://www.ema.europa.eu/docs/en_GB/document_library/Scientific_guideline/2012/0 6/WC500129137.pdf. Accessed September 20, 2016.

5. U.S. Department of Health and Human Services USFDA. Good Pharmacovigilance Practices and Pharmacoepidemiologic Assessment. 2005; http://www.fda.gov/downloads/regulatoryinformation/guidances/ucm126834.pdf. Accessed September 20, 2016.

6. Chiang CE. Congenital and acquired long QT syndrome. Current concepts and management. Cardiology in review. 2004;12(4):222-234.

7. Enger C, Cali C, Walker AM. Serious ventricular arrhythmias among users of cisapride and other QT-prolonging agents in the United States. Pharmacoepidemiology and drug safety. 2002;11(6):477-486.

8. Hennessy S, Leonard CE, Newcomb C, Kimmel SE, Bilker WB. Cisapride and ventricular arrhythmia. British journal of clinical pharmacology. 2008;66(3):375-385.

9. Ray WA, Murray KT, Hall K, Arbogast PG, Stein CM. Azithromycin and the risk of cardiovascular death. N Engl J Med. 2012;366(20):1881-1890.

10. U.S. Department of Health and Human Services USFDA. FDA Drug Safety Communication: Azithromycin (Zithromax or Zmax) and the risk of potentially fatal heart rhythms. 2013; http://www.fda.gov/Drugs/DrugSafety/ucm341822.htm. Accessed September 20, 2016.

11. Denny JC, Miller RA, Waitman LR, Arrieta MA, Peterson JF. Identifying QT prolongation from ECG impressions using a general-purpose Natural Language Processor. Int J Med Inform. 2009;78 Suppl 1:S34-42. 
12. Chung CP, Murray KT, Stein CM, Hall K, Ray WA. A computer case definition for sudden cardiac death. Pharmacoepidemiology and drug safety. 2010;19(6):563-572.

13. Hennessy S, Leonard CE, Freeman CP, et al. Validation of diagnostic codes for outpatient-originating sudden death and ventricular arrhythmia in Medicaid and Medicare claims data. Pharmacoepidemiology and drug safety. 2010;19(6):555-562.

14. Chou HW, Wang JL, Chang CH, Lai CL, Lai MS, Chan KA. Risks of cardiac arrhythmia and mortality among patients using new-generation macrolides, fluoroquinolones, and beta-lactam/beta-lactamase inhibitors: a Taiwanese nationwide study. Clinical infectious diseases : an official publication of the Infectious Diseases Society of America. 2015;60(4):566-577.

15. Rao GA, Mann JR, Shoaibi A, et al. Azithromycin and levofloxacin use and increased risk of cardiac arrhythmia and death. Annals of family medicine. 2014;12(2):121-127.

16. Hanrahan JP, Choo PW, Carlson W, Greineder D, Faich GA, Platt R. Terfenadine-associated ventricular arrhythmias and QTc interval prolongation. A retrospective cohort comparison with other antihistamines among members of a health maintenance organization. Annals of epidemiology. 1995;5(3):201-209.

17. Tamariz L, Harkins T, Nair V. A systematic review of validated methods for identifying ventricular arrhythmias using administrative and claims data. Pharmacoepidemiology and drug safety. 2012;21 Suppl 1:148-153.

18. McDonald KM, Hlatky MA, Saynina O, Geppert J, Garber AM, McClellan MB. Trends in hospital treatment of ventricular arrhythmias among Medicare beneficiaries, 1985 to 1995. American heart journal. 2002;144(3):413-421.

19. Leonard CE, Bilker WB, Newcomb C, Kimmel SE, Hennessy S. Antidepressants and the risk of sudden death and ventricular arrhythmia. Pharmacoepidemiology and drug safety. 2011;20(9):903-913.

20. Leonard CE, Freeman CP, Newcomb CW, et al. Antipsychotics and the Risks of Sudden death and All-Cause Death: Cohort Studies in Medicaid and DuallyEligible Medicaid-Medicare Beneficiaries of Five States. Journal of clinical \& experimental cardiology. 2013;Suppl 10(6):1-9.

21. Kawai VK, Murray KT, Stein CM, et al. Validation of a computer case definition for sudden death in opioid users. BMC Res Notes. 2012;5:473.

22. Schelleman H, Bilker WB, Kimmel SE, et al. Methylphenidate and risk of serious cardiovascular events in adults. Am J Psychiatry. 2012;169(2):178-185. 
23. De Bruin ML, van Hemel NM, Leufkens HG, Hoes AW. Hospital discharge diagnoses of ventricular arrhythmias and cardiac arrest were useful for epidemiologic research. Journal of clinical epidemiology. 2005;58(12):1325-1329.

24. Molokhia M, Pathak A, Lapeyre-Mestre M, et al. Case ascertainment and estimated incidence of drug-induced long-QT syndrome: study in Southwest France. British journal of clinical pharmacology. 2008;66(3):386-395.

25. Zambon A, Polo Friz H, Contiero P, Corrao G. Effect of macrolide and fluoroquinolone antibacterials on the risk of ventricular arrhythmia and cardiac arrest: an observational study in Italy using case-control, case-crossover and case-timecontrol designs. Drug Saf. 2009;32(2):159-167.

26. Svanstrom H, Pasternak B, Hviid A. Use of azithromycin and death from cardiovascular causes. N Engl J Med. 2013;368(18):1704-1712.

27. Inghammar $\mathrm{M}$, Svanstrom $\mathrm{H}$, Melbye $\mathrm{M}$, Pasternak B, Hviid A. Oral fluoroquinolone use and serious arrhythmia: bi-national cohort study. BMJ. 2016;352:i843.

28. Wu CS, Tsai YT, Tsai HJ. Antipsychotic drugs and the risk of ventricular arrhythmia and/or sudden cardiac death: a nation-wide case-crossover study. J Am Heart Assoc. 2015;4(2).

29. Chen HL, Hsiao FY. Domperidone, cytochrome P450 3A4 isoenzyme inhibitors and ventricular arrhythmia: a nationwide case-crossover study. Pharmacoepidemiology and drug safety. 2015;24(8):841-848.

30. Trac MH, McArthur E, Jandoc R, et al. Macrolide antibiotics and the risk of ventricular arrhythmia in older adults. CMAJ. 2016;188(7):E120-129.

31. Johannes CB, Varas-Lorenzo C, McQuay LJ, Midkiff KD, Fife D. Risk of serious ventricular arrhythmia and sudden death in a cohort of users of domperidone: a nested case-control study. Pharmacoepidemiology and drug safety. 2010;19(9):881888.

32. van Noord C, Eijgelsheim M, Stricker BH. Drug- and non-drug-associated QT interval prolongation. British journal of clinical pharmacology. 2010;70(1):16-23.

33. Kannankeril P, Roden DM, Darbar D. Drug-induced long QT syndrome. Pharmacol Rev. 2010;62(4):760-781. 
Table 1.1 Literature search strategy and number of searched studies

\begin{tabular}{|c|c|c|}
\hline & $\begin{array}{l}\text { Search terms } \\
\text { Limits: Humans; English; publication date between 2000/01/01 to } \\
\text { 2016/08/31 }\end{array}$ & $\begin{array}{l}\text { Number of } \\
\text { results }\end{array}$ \\
\hline$\# 1$ & $\begin{array}{l}\text { "Pharmaceutical preparations/adverse effects"[Mesh] OR } \\
\text { "Pharmaceutical preparations/contraindications"[Mesh] OR } \\
\text { "Pharmaceutical preparations/poisoning"[Mesh] OR "Pharmaceutical } \\
\text { preparations/therapeutic use"[Mesh] OR "Pharmaceutical } \\
\text { preparations/toxicity"[Mesh] OR "Pharmaceutical } \\
\text { preparations/therapy"[Mesh] OR "Pharmaceutical } \\
\text { preparations/analysis"[Mesh] OR "Chemical actions and uses/adverse } \\
\text { effects"[Mesh] OR "Chemical actions and } \\
\text { uses/contraindications"[Mesh] OR "Chemical actions and } \\
\text { uses/poisoning"[Mesh] OR "Chemical actions and uses/therapeutic } \\
\text { use"[Mesh] OR "Chemical actions and uses/toxicity"[Mesh] OR } \\
\text { "Chemical actions and uses/therapy"[Mesh] OR "Chemical actions and } \\
\text { uses/analysis"[Mesh] OR "Chemical actions and } \\
\text { uses/epidemiology"[Mesh] OR "Drug toxicity"[Mesh] OR "Diseases } \\
\text { Category/chemically induced"[Mesh] OR "Diseases Category/drug } \\
\text { therapy"[Mesh] OR "Diseases Category/epidemiology"[Mesh] OR } \\
\text { "Validation Studies"[pt] OR "Validation Studies as Topic"[Mesh] OR } \\
\text { "Sensitivity and Specificity"[Mesh] OR "Predictive Value of } \\
\text { Tests"[Mesh] OR "Reproducibility of Results"[Mesh] OR "Predictive } \\
\text { Value”[tw] }\end{array}$ & $2,359,927$ \\
\hline$\# 2$ & $\begin{array}{l}\text { "Premier"[All] OR "Solucient"[All] OR "Cerner"[All] OR } \\
\text { "Ingenix"[All] OR "LabRx"[All] OR "IHCIS"[All] OR } \\
\text { "marketscan"[All] OR "market scan"[All] OR "Medstat"[All] OR } \\
\text { "Thomson"[All] OR "pharmetrics"[All] OR "healthcore"[All] OR } \\
\text { "united healthcare"[All] OR "UnitedHealthcare"[All] OR "UHC"[All] } \\
\text { OR "GPRD"[All] OR "general practice research database"[All] OR } \\
\text { "Research Database"[All] OR "Group Health"[All] OR "HCUP"[All] } \\
\text { OR ("Healthcare Cost"[All] AND "Utilization Project"[All]) OR } \\
\text { ("Health Care Cost"[All] AND "Utilization Project"[All]) OR } \\
\text { "MEPS"[All] OR "Medical Expenditure Panel Survey"[All] OR } \\
\text { "NAMCS"[All] OR "National Hospital Ambulatory Medical Care } \\
\text { Survey"[All] OR "National Ambulatory Medical Care Survey"[All] OR } \\
\text { "NHIS"[All] OR "National Health Interview Survey"[All] OR } \\
\text { "Kaiser"[All] OR "HMO Research"[All] OR "Health Maintenance } \\
\text { Organization"[All] OR "HMO"[All] OR "Cleveland Clinic"[All] OR } \\
\text { "Lovelace"[All] OR "Department of Defense"[All] OR "Henry } \\
\text { Ford"[All] OR ("Denmark"[All] AND "Epidemiology"[All]) OR "i3 } \\
\text { Drug Safety"[All] OR "i3"[All] OR "Aetna"[All] OR "Humana"[All] } \\
\text { OR "Wellpoint"[All] OR "IMS"[All] OR "Intercontinental Marketing } \\
\text { Services"[All] OR "IMS Health"[All] OR "Geisinger"[All] OR "GE } \\
\text { Healthcare"[All] OR "MQIC"[All] OR "PHARMO"[All] OR "Institute } \\
\text { for Drug Outcome Research"[All] OR "Pilgrim"[All] OR "Puget } \\
\text { Sound"[All] OR "Regenstrief"[All] OR "Saskatchewan"[All] OR } \\
\text { "Tayside"[All] OR "MEMO"[All] OR "Medicines Monitoring } \\
\text { Unit"[All] OR "Veterans Affairs"[All] OR "Partners Healthcare"[All] } \\
\text { OR "Mayo Clinic"[All] OR "Rochester Epidemiology"[All] OR "Indiana }\end{array}$ & 521,010 \\
\hline
\end{tabular}




\begin{tabular}{|c|c|c|}
\hline & $\begin{array}{l}\text { Health Information Exchange"[All] OR "Indiana Health"[All] OR } \\
\text { "Intermountain"[All] OR "THIN"[All] OR “The health improvement } \\
\text { network"[All] OR "blue cross"[All] OR "health partners"[All] OR } \\
\text { "health plan"[All] OR "health services"[All] OR "Nationwide Inpatient } \\
\text { Sample"[All] OR "National Inpatient Sample"[All] OR "medicaid"[All] } \\
\text { OR "medicare"[All] OR "MediPlus"[All] OR "Outcome } \\
\text { Assessment"[All] OR "insurance database"[All] OR "insurance } \\
\text { databases"[All] OR "Data Warehouse"[All] OR "ICD-9"[All] OR } \\
\text { “international statistical classification"[All] OR “international } \\
\text { classification of diseases"[All] OR "ICD-10"[All] OR "Database } \\
\text { Management Systems"[Mesh] OR "Medical Records Systems, } \\
\text { Computerized"[Mesh] OR "CPT"[All] OR "Current procedural } \\
\text { terminology"[All] OR "drug surveillance"[All] OR (“claims”[tw] AND } \\
\text { “administrative”[tw]) OR (“data”[tw] AND “administrative”[tw]) OR } \\
\text { "Databases, Factual"[Mesh] OR "Databases as topic"[Mesh] OR } \\
\text { "Medical Record Linkage"[Mesh] OR "ICD-9-CM"[All Fields] OR } \\
\text { "ICD-10-CM"[All Fields] }\end{array}$ & \\
\hline \#3 & $\begin{array}{l}\text { "Clinical Trial"[pt] OR "Editorial"[pt] OR "Letter"[pt] OR "Meta- } \\
\text { Analysis"[pt] OR "Randomized Controlled Trial"[pt] OR "Clinical Trial, } \\
\text { Phase I"[pt] OR "Clinical Trial, Phase II"[pt] OR "Clinical Trial, Phase } \\
\text { III"[pt] OR "Clinical Trial, Phase IV"[pt] OR "Comment"[pt] OR } \\
\text { "Controlled Clinical Trial"[pt] OR "case reports"[pt] OR "Clinical Trials } \\
\text { as Topic"[Mesh] OR "double-blind"[All] OR "placebo-controlled"[All] } \\
\text { OR "pilot study"[All] OR "pilot projects"[Mesh] OR "Review"[pt] OR } \\
\text { "Prospective Studies"[Mesh] }\end{array}$ & $2,995,791$ \\
\hline$\# 4$ & $\begin{array}{l}\text { "Arrhythmias, Cardiac"[Mesh:NoExp] OR "Brugada } \\
\text { Syndrome"[Mesh:NoExp] OR "Cardiac Complexes, Premature"[Mesh] } \\
\text { OR "CommotioCordis"[Mesh:NoExp] OR "Heart Block"[Mesh] OR } \\
\text { "Long QT Syndrome"[Mesh] OR "Parasystole"[Mesh] OR "Pre- } \\
\text { Excitation Syndromes"[Mesh] OR "Tachycardia"[Mesh] OR } \\
\text { "Ventricular Fibrillation"[Mesh] OR "Ventricular Flutter"[Mesh] OR } \\
\text { "Death, Sudden, Cardiac"[Mesh] OR "Torsades de Pointes"[Mesh] OR } \\
\text { "Heart Arrest"[Mesh] OR "Tachycardia, Supraventricular"[Mesh] }\end{array}$ & 55,302 \\
\hline$\# 5$ & Search \#1 AND \#2 & 182,942 \\
\hline$\# 6$ & Search \#5NOT OTHERWISE SPECIFIED \#3 & 120,606 \\
\hline \#7 & Search \#6 AND \#4 & 1,237 \\
\hline
\end{tabular}




\begin{tabular}{|c|c|c|c|c|}
\hline & 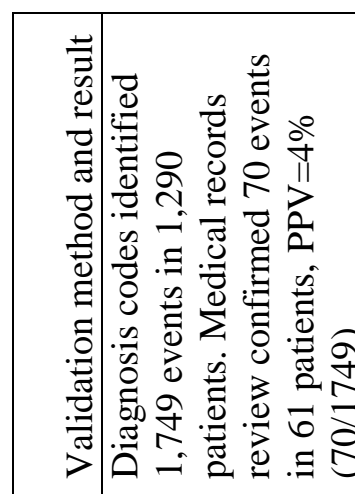 & 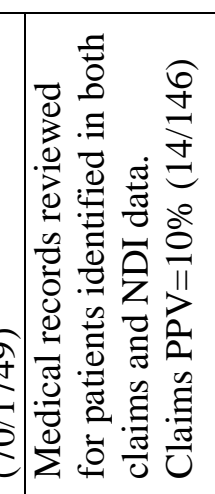 & 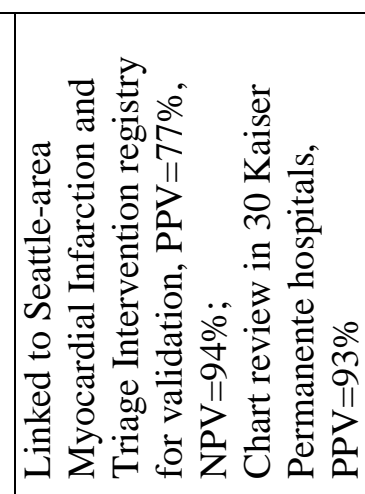 & 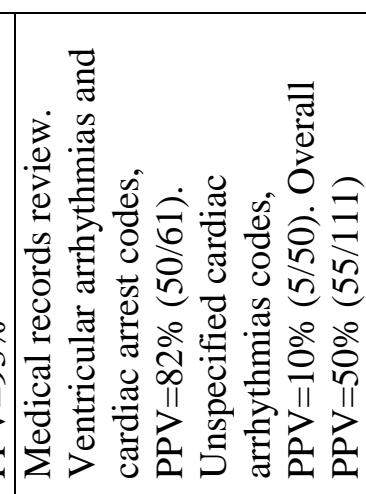 \\
\hline & 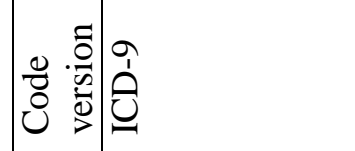 & Oִ & 㝘 & 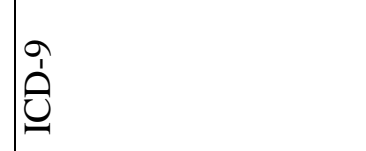 \\
\hline & 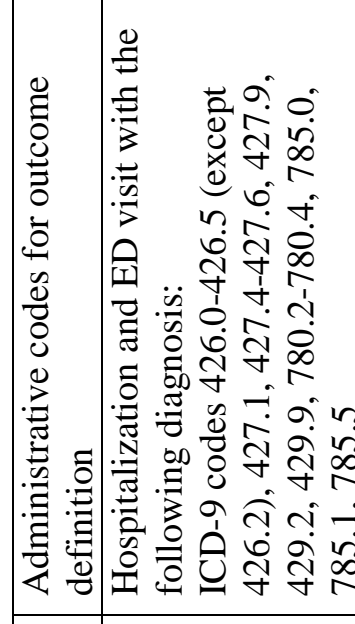 & 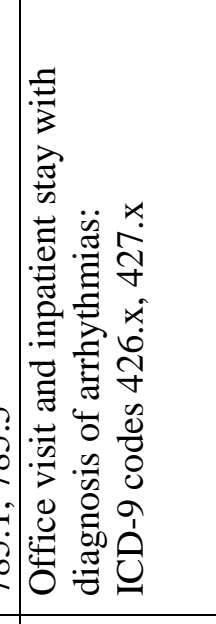 & 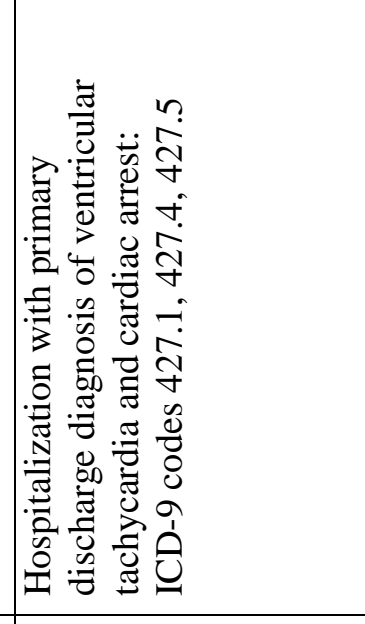 & 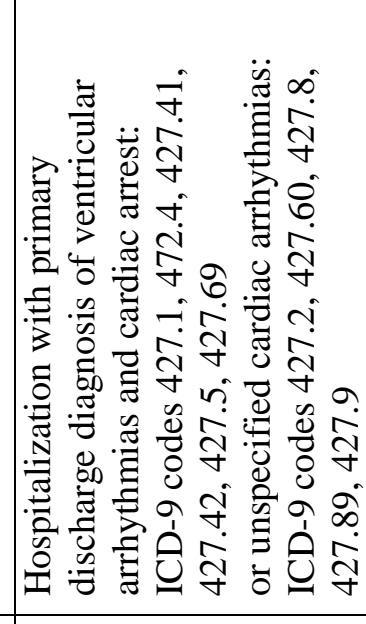 \\
\hline & 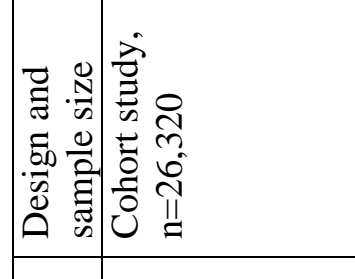 & 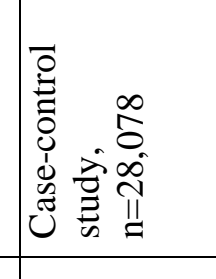 & 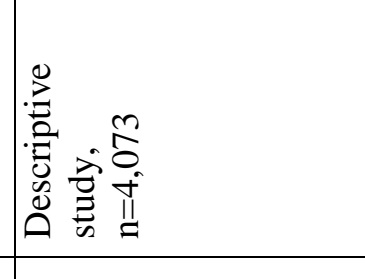 & 喜 \\
\hline & 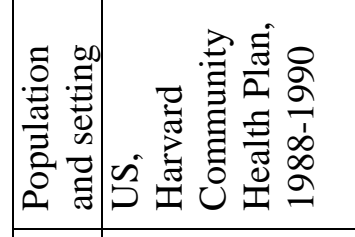 & 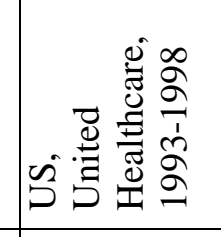 & 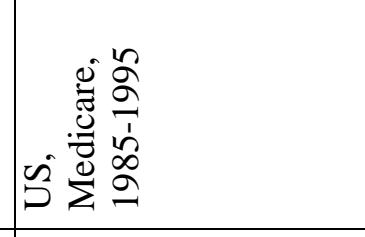 & 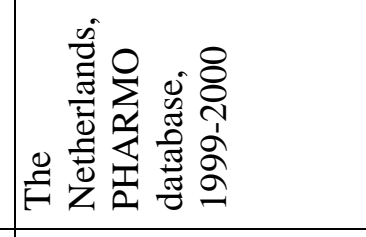 \\
\hline & 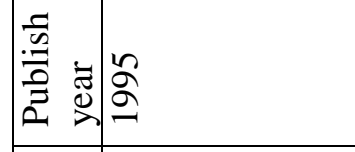 & ర్లి & స్ & 峉 \\
\hline & 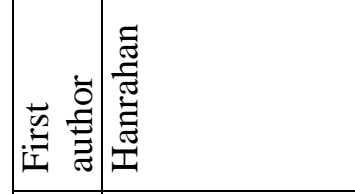 & 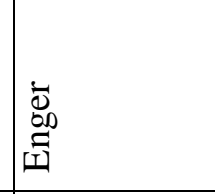 & 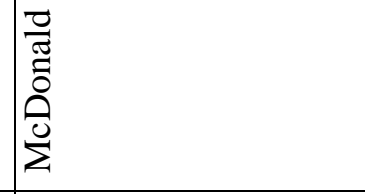 & 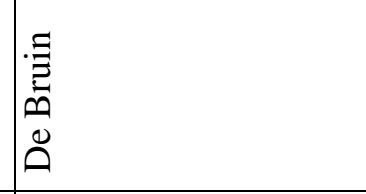 \\
\hline & -1 & N & m & ब \\
\hline
\end{tabular}




\begin{tabular}{|c|c|c|c|c|}
\hline 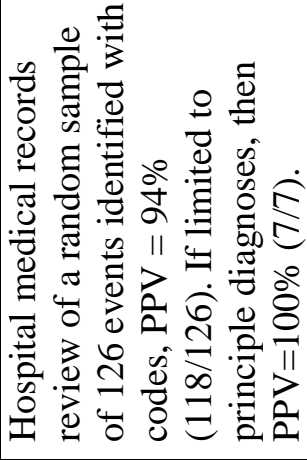 & 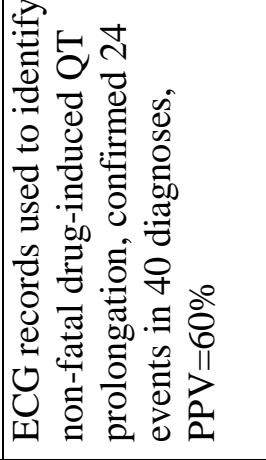 & 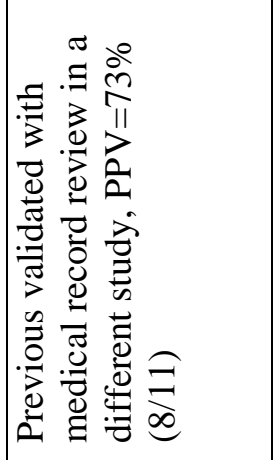 & 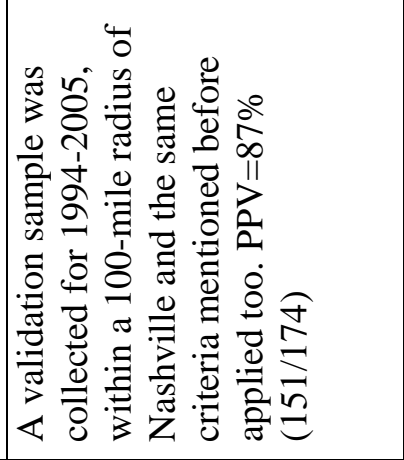 & 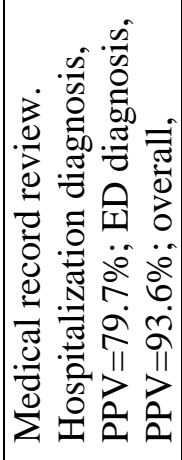 \\
\hline @િ & 옥 & 富 & @ِ & 官 \\
\hline 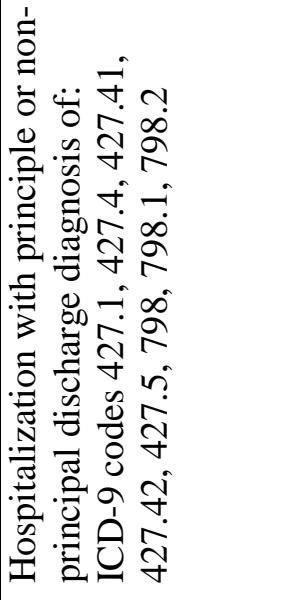 & 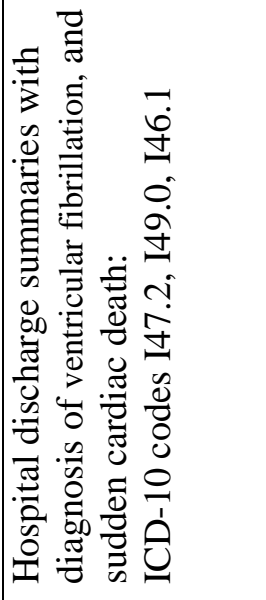 & 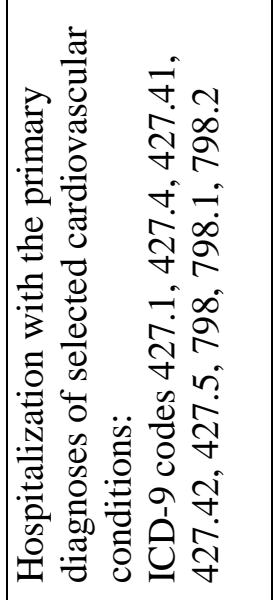 & 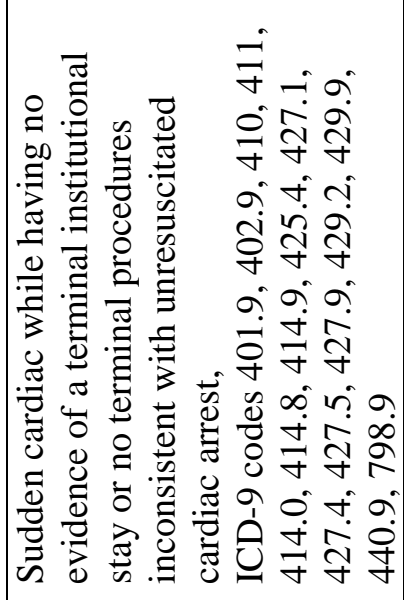 & 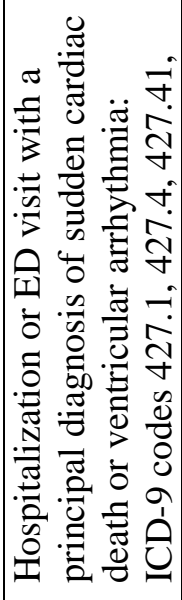 \\
\hline 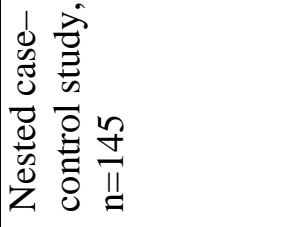 & 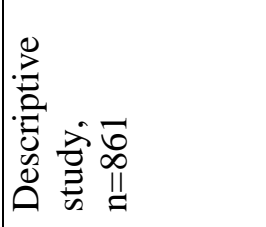 & 完 & 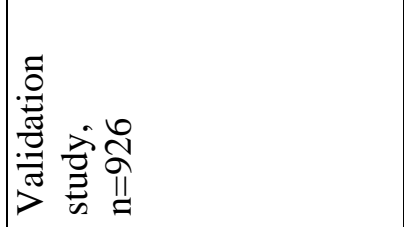 & 总 \\
\hline 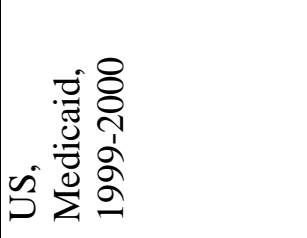 & 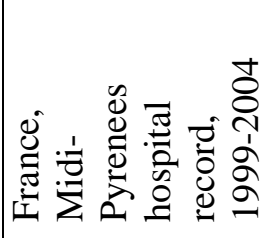 & 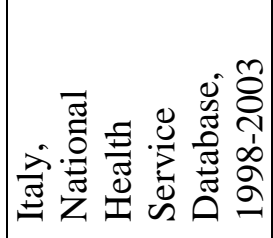 & 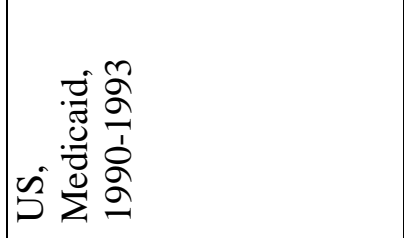 & 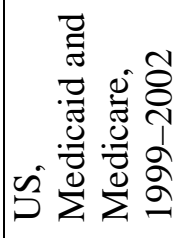 \\
\hline 串 & 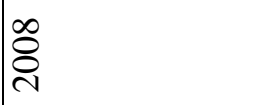 & ஓి & 응 & 옹 \\
\hline 离 & $\frac{.0}{3}$ & 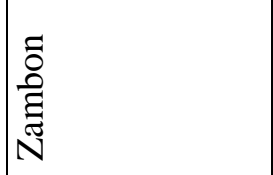 & 亲 & 氙 \\
\hline S & 0 & $\Lambda$ & $\infty$ & $a$ \\
\hline
\end{tabular}




\begin{tabular}{|c|c|c|c|c|}
\hline & 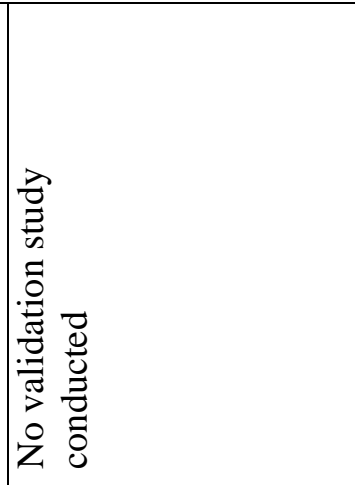 & 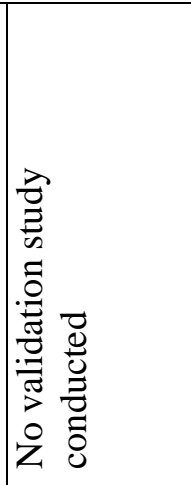 & 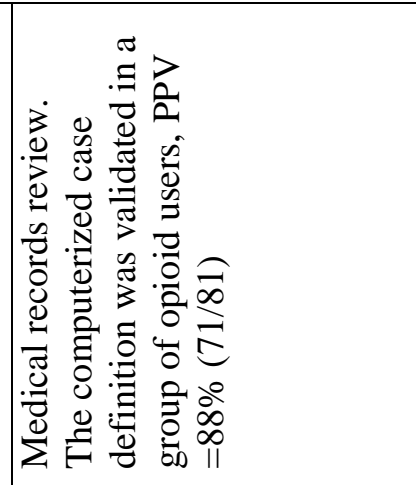 & 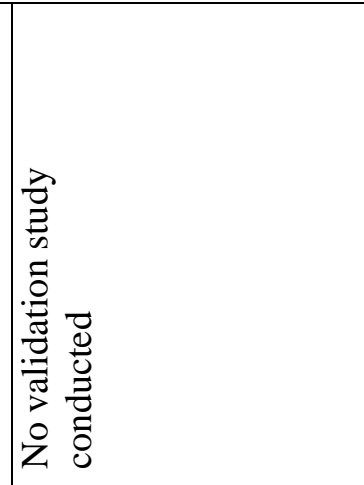 \\
\hline & 仓ِ & 仓̊ & فِ & 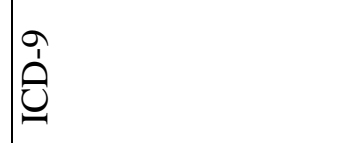 \\
\hline 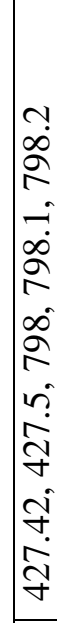 & 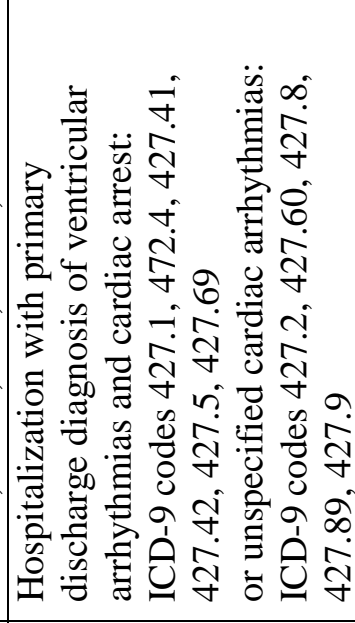 & 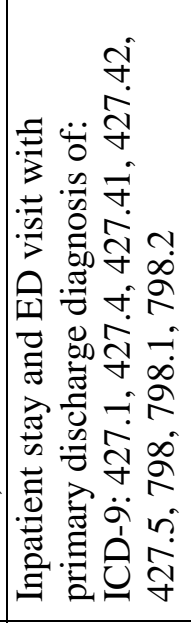 & 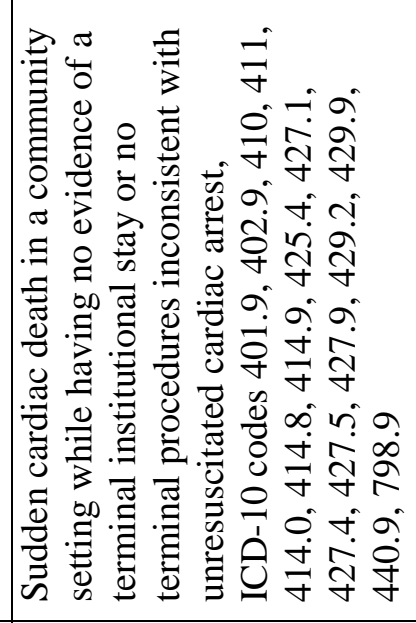 & 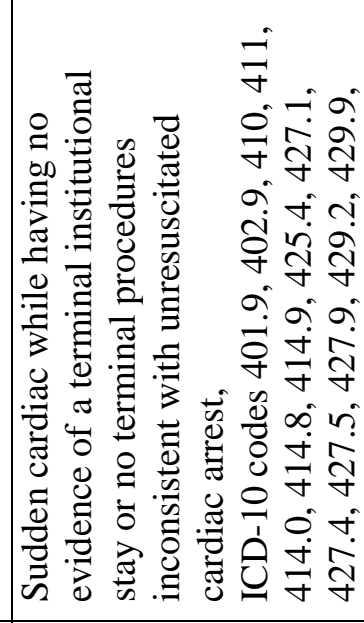 \\
\hline & 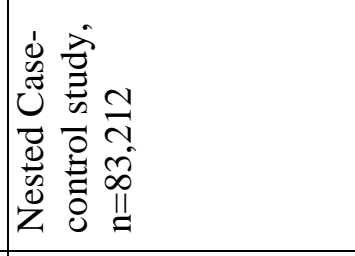 & 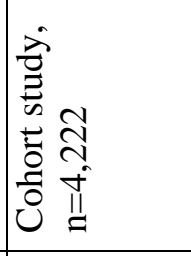 & 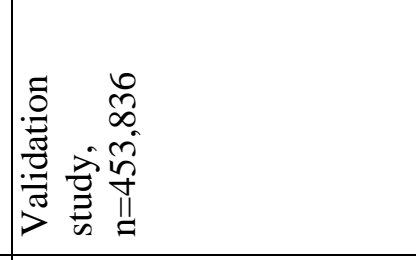 & 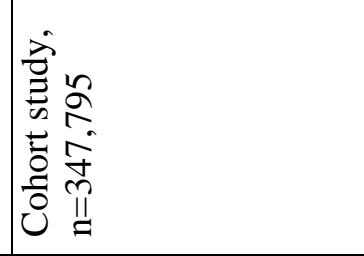 \\
\hline & 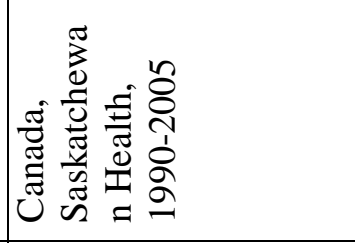 & 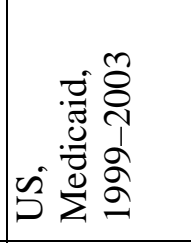 & 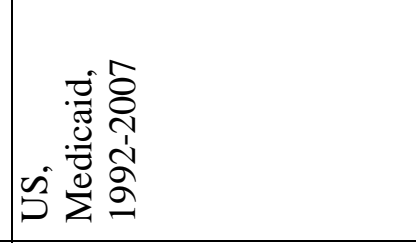 & 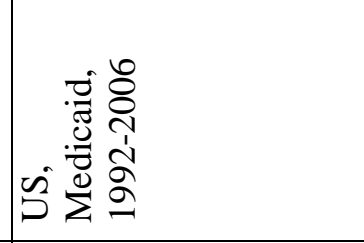 \\
\hline & $\stackrel{\stackrel{\sim}{\sim}}{\stackrel{\sim}{2}}$ & ¿े & $\stackrel{\sim}{\stackrel{\sim}{\sim}}$ & 疋 \\
\hline & 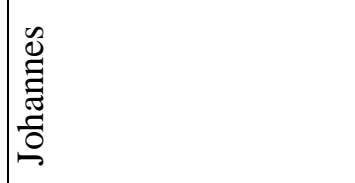 & 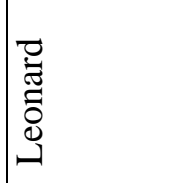 & 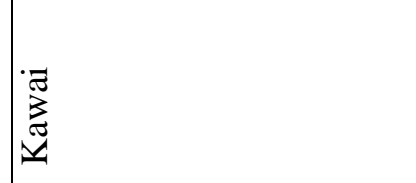 & $\ddot{\mathscr{A}}$ \\
\hline & $\circ$ & $\exists$ & $\approx$ & $\ddot{m}$ \\
\hline
\end{tabular}




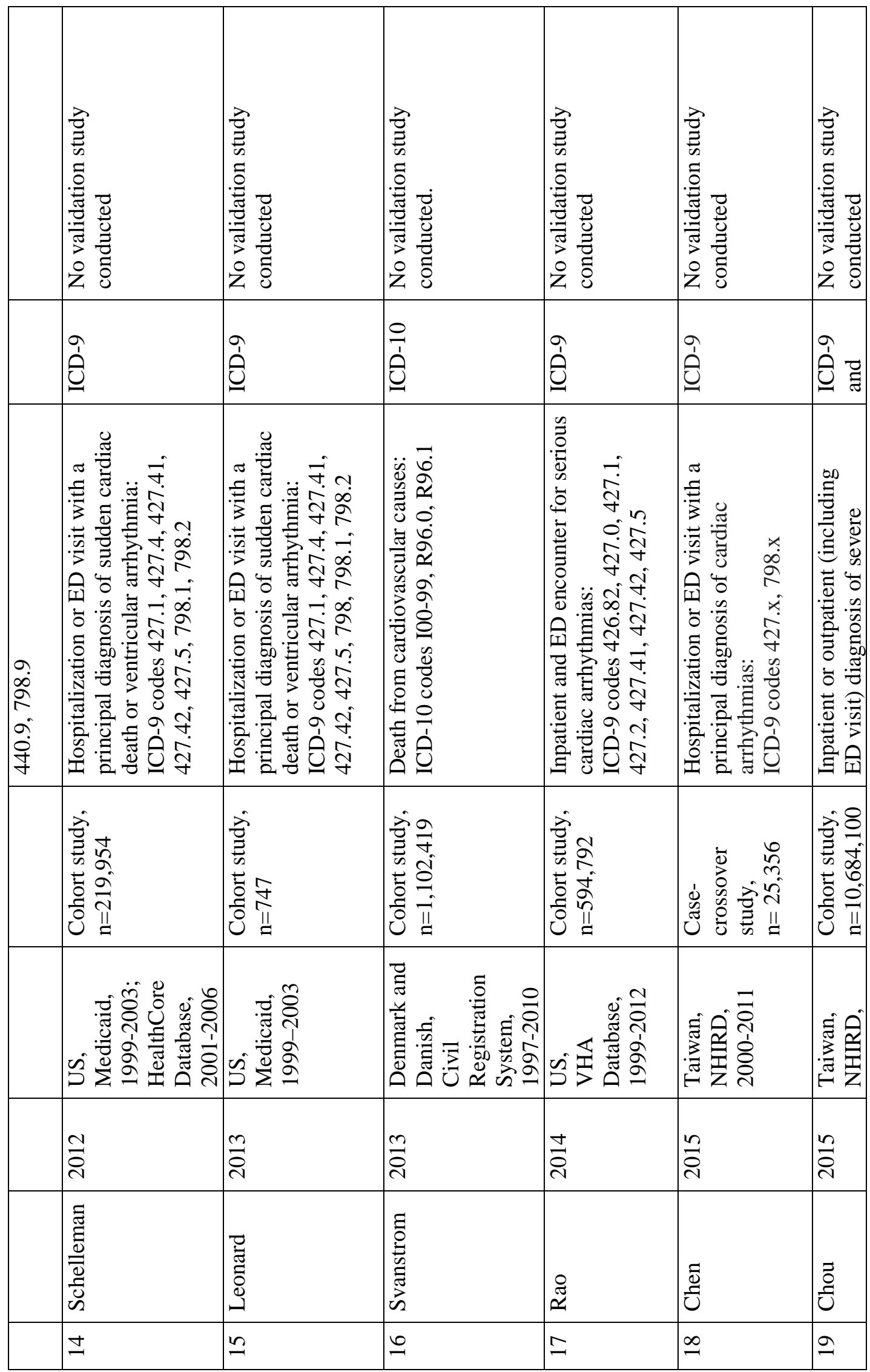




\begin{tabular}{|c|c|c|c|c|}
\hline & 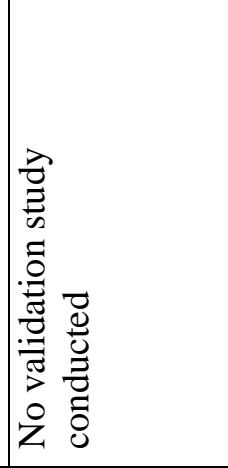 & 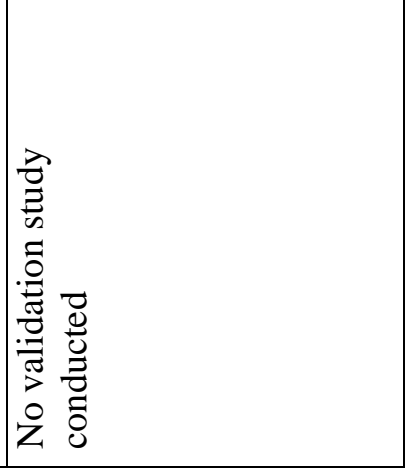 & 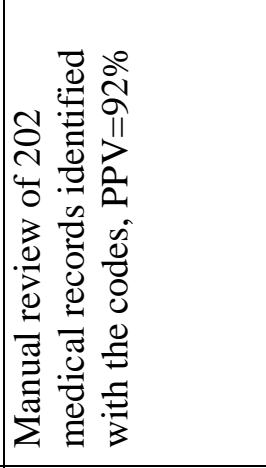 & 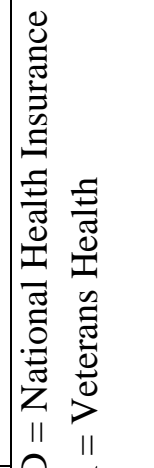 \\
\hline & 畺 & 号 & 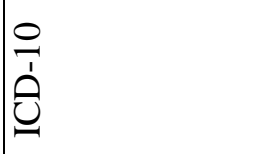 & 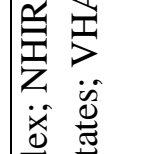 \\
\hline \multirow[t]{2}{*}{ 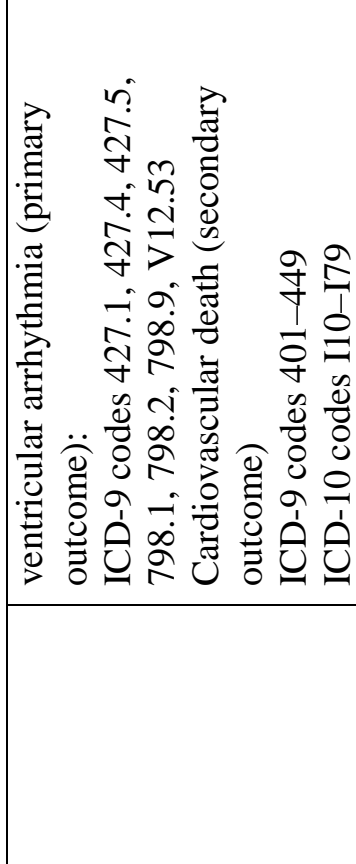 } & 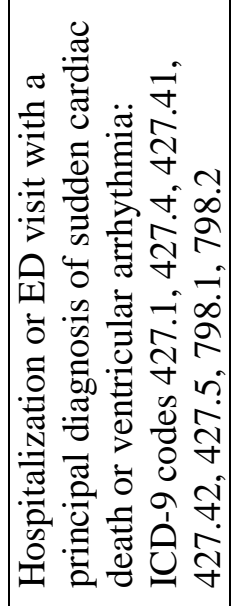 & 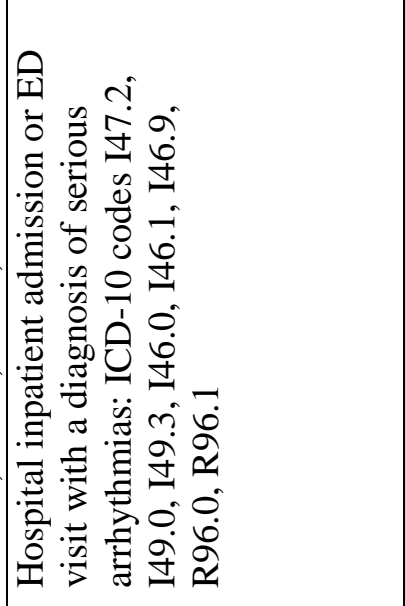 & 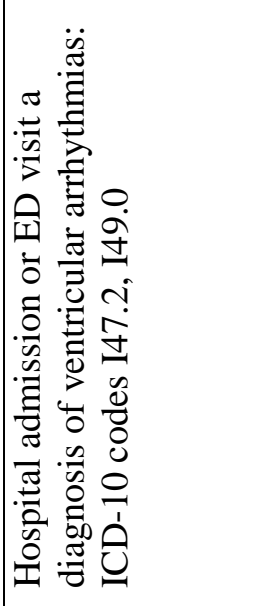 & 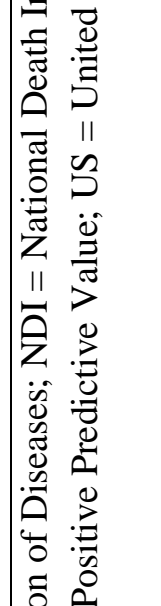 \\
\hline & 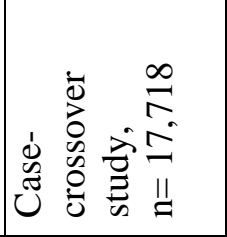 & 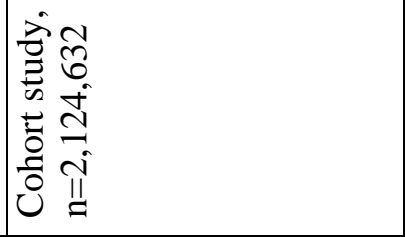 & 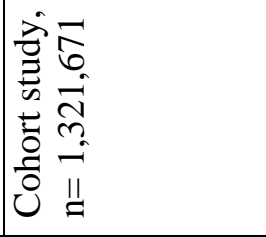 & 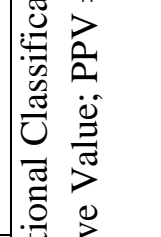 \\
\hline \multirow[t]{4}{*}{$\begin{array}{l}-1 \\
\overline{0} \\
\stackrel{1}{1} \\
\dot{1} \\
0 \\
\stackrel{N}{1}\end{array}$} & 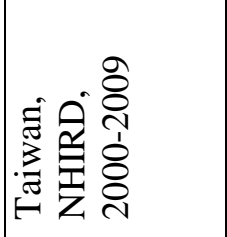 & 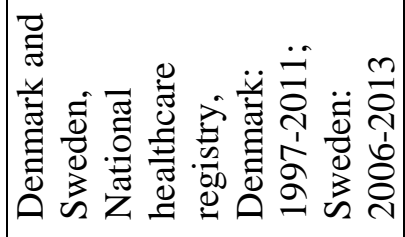 & 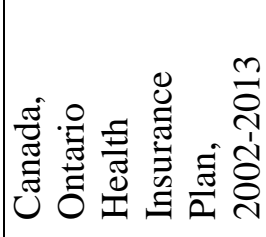 & 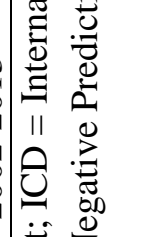 \\
\hline & 늄 & $\stackrel{0}{\stackrel{2}{\sim}}$ & $\begin{array}{l}0 \\
\stackrel{2}{2}\end{array}$ & 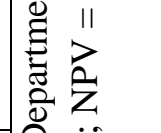 \\
\hline & 3 & 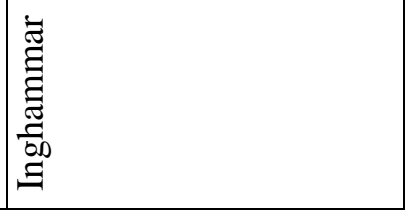 & 曾 & 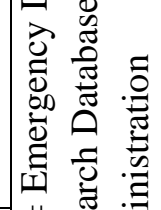 \\
\hline & $\curvearrowright$ & $\ddot{\lambda}$ & $\approx$ & 国 \\
\hline
\end{tabular}


Table 1.3 The most common ICD-9 diagnosis codes for ventricular arrhythmias related to QT prolongation

\begin{tabular}{|l|l|}
\hline ICD-9 code $^{*}$ & \multicolumn{1}{|c|}{ Code description } \\
\hline 427.1 & Paroxysmal ventricular tachycardia \\
\hline 427.4 & Ventricular fibrillation and flutter \\
\hline 427.41 & Ventricular fibrillation \\
\hline 427.42 & Ventricular flutter \\
\hline 427.5 & Cardiac arrest \\
\hline 427.9 & Cardiac dysrhythmia, not otherwise specified \\
\hline $798^{+}$ & Sudden death, cause unknown \\
\hline 798.1 & Instantaneous death \\
\hline 798.2 & $\begin{array}{l}\text { Death occurring in less than 24 hours from onset of } \\
\text { symptoms, not otherwise explained }\end{array}$ \\
\hline
\end{tabular}

ED = Emergency Department; ICD = International Classification of Diseases

* Algorithm used by 5 reviewed studies ${ }^{8,13,19,20,25}$

+ A more specific algorithm (without the general code 798) was used in 2 other studies ${ }^{22,28}$ 
Table 1.4 The most common ICD-9 codes for sudden death related to QT prolongation

\begin{tabular}{|l|l|}
\hline ICD-9 code $^{*}$ & \multicolumn{1}{c|}{ Code description } \\
\hline 401.9 & Essential hypertension, not otherwise specified \\
\hline 402.9 & Hypertensive heart disease, not otherwise specified \\
\hline 410 & Myocardial infarction \\
\hline 411 & Other acute/subacute ischemic heart disease \\
\hline 414.0 & Coronary atherosclerosis \\
\hline 414.8 & Chronic ischemic heart disease, other \\
\hline 414.9 & Chronic ischemic heart disease, unspecified \\
\hline 425.4 & Primary cardiomyopathy, not otherwise specified \\
\hline 427.1 & Paroxysmal ventricular tachycardia \\
\hline 427.4 & Ventricular fibrillation and flutter \\
\hline 427.5 & Cardiac arrest \\
\hline 427.9 & Cardiac dysrhythmia, not otherwise specified \\
\hline 429.2 & Cardiovascular arteriosclerosis \\
\hline 429.9 & Cardiovascular disease, not otherwise specified \\
\hline 440.9 & Atherosclerosis, generalized and unspecified \\
\hline 798.9 & Unattended death \\
\hline
\end{tabular}

ICD = International Classification of Diseases

* Algorithm used by 3 reviewed studies $9,12,21$ 
Figure1.1 Flow chart of literature search and selection process

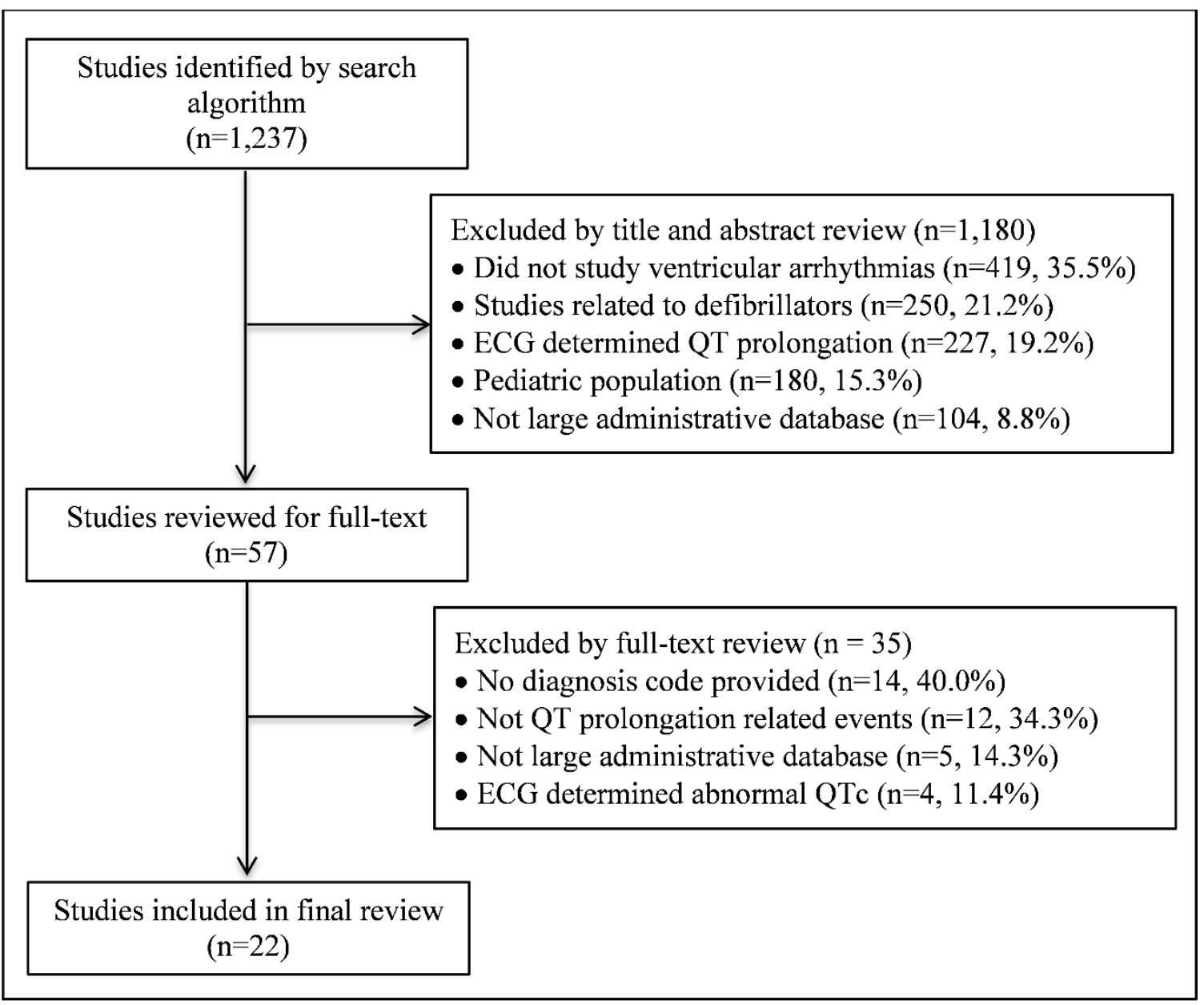


Manuscript II. Concomitant use of QT-prolonging medications and the risk of ventricular arrhythmia and sudden death

Yizhou Ye, M.S. ${ }^{1}$, Kerry L. LaPlante, Pharm.D. ${ }^{1,2}$, E P. Larrat, Ph.D. ${ }^{1}$, and Aisling R.

Caffrey, Ph.D., M.S. ${ }^{1,2,3}$

1. College of Pharmacy, Department of Pharmacy Practice, University of Rhode Island, 7 Greenhouse Rd, Kingston, RI, United States

2. Veterans Affairs Medical Center, Infectious Diseases Research Program and Center of Innovation in Long Term Services and Supports, Providence, RI, United States

3. School of Public Health, Brown University, Providence, RI, United States

Corresponding Author: Aisling R. Caffrey, PhD, MS, Assistant Professor, University of Rhode Island; 7 Greenhouse Road, Kingston, RI 02881; office: (401) 874-5320; e-mail: Aisling_Caffrey@uri.edu

Abstract word count: 319

Manuscript word count: 2569

Table/figure count: $5 / 0$

Funding: Unfunded.

Keywords: concomitant drug use, drug-induced QT prolongation, drug safety, retrospective study, sudden death, ventricular arrhythmia

Target journal: Antimicrobial Agents and Chemotherapy

Publication status: Preparing for publication 


\section{ABSTRACT}

Background: A major drug safety concern associated with hundreds of medication is the potential for QT interval prolongation. However, the real-world impact of taking multiple medications with QT prolongation potential has not been widely studied since events related to this adverse event are rare.

Objective: To investigate the risk for ventricular arrhythmia or sudden death associated with concomitant prescriptions of QT-prolonging drugs.

Methods: A retrospective case-control study was conducted using an administrative health claims database from a large national insurer. Cases of ventricular arrhythmia or sudden death between 2011 and 2013 were matched with four controls of the same age, sex, and geographic region. Concomitant drug use was defined as exposure to an antibiotic with QT-prolonging potential and one other medication carrying the same risk with overlapping durations of prescriptions. Proximal drug use was defined as a gap no larger than 15 days between durations of a QT-prolonging antibiotic and one other QT-prolonging drug. Adjusted odds ratios were estimated for concomitant and proximal QT-prolonging medication use and other risk factors using conditional logistic regression.

Results: We identified 4,612 cases of ventricular arrhythmia and sudden death and 18,448 controls. Concomitant QT medication use was a strong predictor of ventricular arrhythmia and sudden death (adjusted odds ratio [aOR] 2.91; 95\% confidence interval [CI] 1.19-7.08). Proximal QT medication use was also predictive of ventricular arrhythmia and sudden death (aOR 2.20; 95\% CI 1.23-3.40). Our study confirmed 
several known predictors, such as hypokalemia and cardiac arrhythmia, and also identified several new predictors, including higher Charlson Comorbidity Index. Risk of ventricular arrhythmia or sudden death increased as the number of exposures to QT medications increased in the 10 days prior to the event.

Conclusion: In this privately-insured adult population, concomitant and proximal use of QT-prolonging antibiotics with other QT medications was predictive of ventricular arrhythmia or sudden death. In clinical practice, exposure to multiple QT-prolonging medications should be avoided or closely monitored when necessary. 


\section{BACKGROUND}

Macrolide and fluoroquinolone antibiotics are among the most frequently prescribed drugs that are associated QT prolongation, which can lead to torsades de pointes (TdP) arrhythmia and sudden death. ${ }^{1-6}$ The risk is dose-dependent, therefore concomitant use of multiple QT-prolonging medications (QT medication) may pose a greater threat than singular use. TdP is also associated with other risk factors such as

older age, female sex, hypokalemia, history of heart diseases, and renal impairment. ${ }^{7,8}$

Pharmacokinetic interactions among QT-prolonging agents are well-studied. Numerous QT drugs are metabolized by the same group of cytochrome isoenzymes (mostly CYP3A4). ${ }^{9,10}$ When exposed together, one drug will serve as the other's metabolic enzyme inhibitor and cause drug accumulation which further increases the risk of drug-induced QT prolongation, a concentration-related event. In addition, pharmacodynamics is another mechanism for increased risk as the medications can serve as each other's synergist or antagonist. ${ }^{9,11}$ Some macrolides and fluoroquinolones are also well known for pharmacokinetic drug-drug interactions. ${ }^{12}$ Clarithromycin, erythromycin, and ciprofloxacin are all strong inhibitors of cytochrome P450 isoenzymes (CYPs). ${ }^{9}$ Thus, their safety profile may outweigh any potential benefits when used concomitantly, especially if one of the drugs is prescribed in high doses. When such interactions are suspected, available therapeutic alternatives should be considered in order to reduce the risk of ventricular arrhythmia and sudden death. $^{13}$ 
This study sought to quantify predictors of ventricular arrhythmia and sudden death associated with drug-induced QT prolongation, including concomitant exposure to one QT-prolonging antibiotic (QT antibiotic) and one other QT-prolonging medication (other QT medication) with similar pro-arrhythmic potential, in a national, privately-insured population. 


\section{METHODS}

To achieve the study objective, a retrospective matched case-control study was conducted to assess risk factors of ventricular arrhythmia and sudden death, including concomitant QT medication exposures.

\section{Data source}

We utilized the de-identified Optum Clinformatics ${ }^{\mathrm{TM}}$ DataMart database (OptumInsight, Eden Prairie, MN) for this study, which is an administrative health claims database from a large national insurer. We utilized member eligibility files, medical claims, confinement claims, and pharmacy claims to conduct this study.

\section{Study population}

From the data source, we identified cases of severe ventricular arrhythmias and/or sudden death from January 1, 2011 to December 31, 2013. The cases were defined as having a primary hospitalization diagnosis or first-listed emergency department (ED) visit for ventricular arrhythmia, cardiac arrest, sudden death, or instantaneous death (International Classification of Diseases, Ninth Revision, Clinical Modification [ICD-9-CM] codes: 427.1, 427.4, 427.41, 427.42, 427.5, 798, 798.1, and 798.2). This outcome definition was validated in previous studies with positive predictive values (PPVs) of over $80 \% .{ }^{14,15}$ Four controls were matched to each case by exact age in years, sex, and geographic region of the patient.

The diagnosis date was defined as the case index date $\left(\mathrm{t}_{0}\right)$ and each matched control was assigned their case's index date. Included patients were at least 18 years of 
age on index date and had at least 365 days of continuous enrollment prior to the index date to identify relevant predictors, including medical history and previous medication exposures. We excluded patients that had congenital cardiovascular anomalies since these patients had a higher baseline risk for sudden death compared to the rest of the population. Patients with unknown age or sex were also excluded from the study.

\section{Independent Predictors}

Medications with risk of QT prolongation were identified from pharmacy claims, and categorized by class: QT antibiotics (macrolides or fluoroquinolones), antidepressants, antipsychotics, antihistamines, and antiarrhythmics. ${ }^{16-18}$ The list of medications assessed can be found in Appendix A. Prescription duration was calculated from the fill date and days of supply. A patient was considered having concomitant drug exposure if the patient: 1) received any QT antibiotic 30 days prior to index date, 2) also received other QT medications (antidepressants, antipsychotics, antihistamines, or antiarrhythmics), and 3) the prescriptions of the QT antibiotic and the other QT medication overlapped by at least one day. ${ }^{19}$ In a sensitivity analysis, rather than concomitant use, a proximal use definition of within \pm 15 days was applied. We also identified the number of QT medications used by each patient 10 days prior to the index date. The value was categorized as $0,1,2$, and $\geqslant 3$ to assess the risk of multiple QT medications in that time frame, regardless of overlap.

Other potential predictors included the Charlson Comorbidity Index, calculated from medical and confinement claims 365 days prior to the index date. ${ }^{20,21}$ We also assessed other known risk factors for ventricular arrhythmia or sudden death in the 
365 days prior to the index date. ${ }^{7,12,22,23}$ Due to potential cardiac risks, clarithromycin, erythromycin, ciprofloxacin, and levofloxacin dose adjustment is recommended for patients with hypokalemia and renal impairment. ${ }^{24}$ Therefore, the effect of hypokalemia and chronic kidney disease were assessed as risk factors in our study. Furthermore, relevant cardiac comorbidities were identified from diagnosis (Appendix A). ${ }^{7,23,25}$

\section{Statistical Analysis}

For direct comparisons between cases and controls, the Pearson Chi-square $(\chi 2)$ test was utilized for categorical variables. For categorical variables that did not meet assumptions for $\chi 2(\mathrm{n}<5)$, the Fisher's exact test was used. T-test was used for comparing continuous normally distributed demographic variables. Conditional logistic regression was used to identify independent predictors associated being a case using an iterative, manual backward elimination approach. ${ }^{26}$ We used univariable analyses to select potential significant independent predictors that had a p-value < 0.05. Selected variables were tested for collinearity and correlated variables were selected based on clinical significance. Then a multivariable conditional logistic regression model containing all covariates identified in the univarible analyses was fit to assess the importance of each covariate and non-significant variables (p-value > 0.05) were removed one-by-one until only significant predictors remained in the model. Adjusted odds ratios (AORs) and associated 95\% confidence intervals (CI) were reported from the final conditional logistic regression model. 
All statistical analyses were performed using SAS Enterprise Guide, version 7.1 (SAS Institute Inc., Cary, NC, USA). This study was reviewed and approved as exempt by the University of Rhode Island's Institutional Review Board. 


\section{RESULTS}

We identified 4,612 patients who had ventricular arrhythmia or sudden death from January 1, 2011 through December 31, 2013 and 18,448 controls matched on age, sex, and geographic region. The matched cases and controls had identical distributions for the matching variables (Table 2.1). The comorbidity burden in the cases was higher than the controls. The mean Charlson comorbidity index was 2.8 (standard deviation [SD] 3.1) among cases and 0.5 (SD 1.3) among controls. Cases were significantly $(\mathrm{p}<0.0001)$ more likely to have a history of cardiovascular diseases than controls as well as myocardial infarction (10.3\% cases, $1.1 \%$ controls), congestive heart failure (31.6\% cases, $2.5 \%$ controls), cardiac arrhythmia (40.0\% cases, 5.3\% controls), and heart valve disease (21.7\% cases, 3.3\% controls), diabetes with complications (11.6\% cases, $2.0 \%$ controls) and hypokalemia (7.4\% cases, $0.9 \%$ controls).

In the study population, 1,862 patients had exposure to QT-prolonging antibiotics 30 days prior to the event date. Among these patients, 404 were exposed to macrolide antibiotics and 1,458 were exposed to fluoroquinolone antibiotics, while none were exposed to both classes of medications in that 30 day period. Only 37 patients in our study population had concomitant exposures (Table 2.1). Proximal QTprolonging medication use, where medication durations occurred within 15 days of each other, was observed in 81 patients. Among the 23,060 patients included in this study, 20,461 (88.7\%) did not use any QT-prolonging medications in the 10 days prior 
to index date, while 1,807 (7.8\%) patients used one QT-prolonging medication, 619 (2.7\%) used two, and $173(0.8 \%)$ used three or more.

\section{Predictors}

Patients with concomitant QT-prolonging medication use (QT antibiotic + other QT medication) had a 3-fold increase in the odds of ventricular arrhythmia or sudden death (adjusted odds ratio [aOR] 2.91; 95\% CI 1.19-7.08, Table 2.2). Proximal QT-prolonging medication use within 15 days was also a strong predictor of ventricular arrhythmia or sudden death (aOR 2.2, 95\% CI 1.23-3.99, Table 2.3). The strongest predictors of ventricular arrhythmia or sudden death for both concomitant (Table 2.2) and proximal (Table 2.3) use were the Charlson Comorbidity Index and a history of cardiac arrhythmias. Similar predictors of comorbidity burden and specific cardiac conditions were identified when evaluating concomitant or proximal QTprolonging medication exposures. History of palpitation, congestive heart failure, chronic pulmonary disease, cardiomyopathy, and hypokalemia were also identified as independent predictors in the multivariable models. Patients who had diabetes without complications, myocardial infarction, mild liver disease, chronic pulmonary disease, heart murmurs, and heart valve disease all had an increased odds of being a case.

The use of any number of QT-prolonging medications in the 10 days prior to the event was predictive of ventricular arrhythmia or sudden death. Compared with patients who did not used any QT-prolonging medication, those who were exposed to 1, 2, or more than 2 ( $\geq 3$ ) QT drugs had $162 \%, 157 \%$, and $211 \%$ higher odds of developing ventricular arrhythmia or sudden death (Table 2.4). 


\section{DISCUSSION}

In our study, we identified that concomitant exposure to one QT antibiotic and another QT-prolonging drug predicted ventricular arrhythmia and sudden death.

Recent exposure to any QT-prolonging medication within 10 days was also predictive, and the strength of this relationship increased with increasing number of QTprolonging medications. To our knowledge, this is the first observational study to assess the association of ventricular arrhythmia or sudden death with concomitant use of QT-prolonging medications in a large, national, commercially insured population.

There have been extensive pharmacokinetic and pharmacodynamic research on interactions between QT medications, and between QT-prolonging medications and other risk factors for ventricular arrhythmia. ${ }^{8,9,12,27,28}$ Case reports have also reported QT prolongation among patients exposed to multiple drugs with known risk of QT prolongation and TdP. ${ }^{29-31}$ Few studies have assessed the effects of receiving multiple QT-prolonging medications in retrospective studies. Several previous studies assessed the prevalence of concomitant use of antipsychotics that prolong QT interval. ${ }^{24,32} \mathrm{~A}$ study using claims from a pharmacy benefits manager in the United States found that 51\% of QT-prolonging antipsychotic users filled prescriptions for another QT

medication with an overlap of at least 1 day in the durations of the two prescriptions. ${ }^{32}$ Another study in a tertiary care hospital in Switzerland found that 38.7\% $(1,332 / 3,444)$ administered courses of macrolides or fluoroquinolones had concomitant use of additional QT-prolonging drugs. ${ }^{24}$ 
There have been general warnings regarding concomitant use of QTprolonging drugs and contraindications noted on many drug labels. These warnings may have contributed to the low prevalence of concomitant QT medication use in our cases and controls. During the study period, only 37 patients (0.2\%) had concomitant use (QT antibiotic and other QT medication) within the 30-day window prior to ventricular arrhythmia or sudden death. Our sensitivity analysis also only identified 81 patients (0.4\%) with proximal use (QT antibiotic and other QT medication). Despite this infrequent use, concomitant (aOR 2.91) and proximal (aOR 2.20) use of QT medications was a strong predictor of ventricular arrhythmia and sudden death. These results suggest co-administration of medications with pro-arrhythmic potential should be avoided in clinical practice as the subsequent risk of ventricular arrhythmia and sudden death was high.

Several risk factors for QT prolongation and outcomes of this have been identified in previous studies. ${ }^{7,8,12,17,22}$ Our study confirms several risk factors for QT prolongation, including hypokalemia, cardiac arrhythmia, cardiomyopathy, coronary artery disease, heart murmurs, heart valve disease, and palpitation. Our study provided the magnitude of these risk factors in a large national retrospective population. We also identified several new predictors in our population, such as myocardial infarction, diabetes without complications, metastatic solid tumor, congestive heart failure, chronic pulmonary disease, and mild liver disease.

This study, however, is not without limitations. In lieu of actual drug administration information, we relied on dispensed prescriptions records from an administrative claims database as our measure of exposure. Therefore, inpatient 
exposure to injected or intravenously administered QT-prolonging medications could not be assessed. In addition, several risk factors for ventricular arrhythmia and sudden death, such as smoking status and body mass index, were also not available from our data source. Other over-the-counter medications with QT-prolonging potential, such as diphenhydramine, ${ }^{33}$ are not captured by administrative data. Therefore, patients categorized as unexposed to QT-prolonging medications may have been misclassified. Finally, due to the nature of an administrative claims database, we relied on diagnosis codes for ventricular arrhythmia and sudden death. Therefore, had these events occurred but not resulted in claims with these specific diagnoses, misclassification of case-control status could have also occurred. We did utilize an outcome definition that has been validated in several populations. ${ }^{14,15}$

Closer monitoring should be implemented when concomitant exposure of QTprolonging medications is necessary. Further research is still required for class specific combinations, dose-related effects, and interactions between medications and medical conditions. In our retrospective analysis, we only looked at antibiotics, antiarrhythmics, antihistamines, antidepressants, and antipsychotics while there are many other drugs with pro-arrhythmic potential (known, possible, and conditional according to CredibleMeds), which could be expanded in future studies. ${ }^{33}$ Additionally, the interaction between QT medications and medical conditions is important area of future study as the physiologic and pathologic changes in patients might alter the metabolism and excretion of QT medications and disturb the balance of ions for normal ventricular myocyte function. ${ }^{34,35}$ 


\section{CONCLUSION}

We confirmed several known predictors of QT prolongation in this privatelyinsured adult population, including hypokalemia, cardiac arrhythmia, cardiomyopathy, coronary artery disease, heart murmurs, heart valve disease, palpitation, myocardial infarction, diabetes without chronic complication, metastatic solid tumor, congestive heart failure, chronic pulmonary disease, and mild liver disease. The concomitant exposure to a QT-prolonging antibiotic and other QT medication was predictive of ventricular arrhythmia or sudden death. Risk of ventricular arrhythmia or sudden death increased as the number of exposures to QT medications increased in the 10 days prior to the event. In clinical practice, exposure to multiple QT-prolonging medications should be avoided or closely monitored when necessary. 


\section{REFERENCES}

1. Briasoulis A, Agarwal V, Pierce WJ. QT prolongation and torsade de pointes induced by fluoroquinolones: infrequent side effects from commonly used medications. Cardiology. 2011;120(2):103-110.

2. Owens RC, Jr., Nolin TD. Antimicrobial-associated QT interval prolongation: pointes of interest. Clinical infectious diseases : an official publication of the Infectious Diseases Society of America. 2006;43(12):1603-1611.

3. Ray WA, Murray KT, Hall K, Arbogast PG, Stein CM. Azithromycin and the risk of cardiovascular death. N Engl J Med. 2012;366(20):1881-1890.

4. U.S. Department of Health and Human Services USFDA. FDA Drug Safety Communication: Azithromycin (Zithromax or Zmax) and the risk of potentially fatal heart rhythms. 2013; http://www.fda.gov/Drugs/DrugSafety/ucm341822.htm. Accessed September 20, 2016.

5. Liu HH. Safety profile of the fluoroquinolones: focus on levofloxacin. Drug Saf. 2010;33(5):353-369.

6. Mehrzad R, Barza M. Weighing the adverse cardiac effects of fluoroquinolones: A risk perspective. J Clin Pharmacol. 2015;55(11):1198-1206.

7. $\quad$ Roden DM. Drug-induced prolongation of the QT interval. N Engl J Med. 2004;350(10):1013-1022.

8. Yap YG, Camm AJ. Drug induced QT prolongation and torsades de pointes. Heart. 2003;89(11):1363-1372.

9. Dresser GK, Spence JD, Bailey DG. Pharmacokinetic-pharmacodynamic consequences and clinical relevance of cytochrome P450 3A4 inhibition. Clin Pharmacokinet. 2000;38(1):41-57.

10. Chen HL, Hsiao FY. Domperidone, cytochrome P450 3A4 isoenzyme inhibitors and ventricular arrhythmia: a nationwide case-crossover study. Pharmacoepidemiology and drug safety. 2015;24(8):841-848.

11. Aerssens J, Paulussen AD. Pharmacogenomics and acquired long QT syndrome. Pharmacogenomics. 2005;6(3):259-270.

12. van Noord C, Eijgelsheim M, Stricker BH. Drug- and non-drug-associated QT interval prolongation. British journal of clinical pharmacology. 2010;70(1):16-23.

13. Isbister GK, Page CB. Drug induced QT prolongation: the measurement and assessment of the QT interval in clinical practice. British journal of clinical pharmacology. 2013;76(1):48-57. 
14. Hennessy S, Leonard CE, Freeman CP, et al. Validation of diagnostic codes for outpatient-originating sudden cardiac death and ventricular arrhythmia in Medicaid and Medicare claims data. Pharmacoepidemiology and drug safety. 2010;19(6):555562.

15. De Bruin ML, van Hemel NM, Leufkens HG, Hoes AW. Hospital discharge diagnoses of ventricular arrhythmias and cardiac arrest were useful for epidemiologic research. Journal of clinical epidemiology. 2005;58(12):1325-1329.

16. Astrom-Lilja C, Odeberg JM, Ekman E, Hagg S. Drug-induced torsades de pointes: a review of the Swedish pharmacovigilance database. Pharmacoepidemiology and drug safety. 2008;17(6):587-592.

17. Isbister GK. Risk assessment of drug-induced QT prolongation. Aust Prescr. 2015;38(1):20-24.

18. Nachimuthu S, Assar MD, Schussler JM. Drug-induced QT interval prolongation: mechanisms and clinical management. Ther Adv Drug Saf. 2012;3(5):241-253.

19. Tobi H, Faber A, van den Berg PB, Drane JW, de Jong-van den Berg LT. Studying co-medication patterns: the impact of definitions. Pharmacoepidemiology and drug safety. 2007;16(4):405-411.

20. Quan H, Li B, Couris CM, et al. Updating and validating the Charlson comorbidity index and score for risk adjustment in hospital discharge abstracts using data from 6 countries. Am J Epidemiol. 2011;173(6):676-682.

21. Charlson M, Szatrowski TP, Peterson J, Gold J. Validation of a combined comorbidity index. Journal of clinical epidemiology. 1994;47(11):1245-1251.

22. Paulussen AD, Aerssens J. Risk factors for drug-induced long-QT syndrome. Neth Heart J. 2005;13(2):47-56.

23. Kannankeril P, Roden DM, Darbar D. Drug-induced long QT syndrome. Pharmacol Rev. 2010;62(4):760-781.

24. Niedrig D, Maechler S, Hoppe L, Corti N, Kovari H, Russmann S. Drug safety of macrolide and quinolone antibiotics in a tertiary care hospital: administration of interacting co-medication and QT prolongation. Eur J Clin Pharmacol. 2016;72(7):859-867.

25. Roden DM. Clinical practice. Long-QT syndrome. N Engl J Med. 2008;358(2):169-176.

26. Hosmer D.W LS, Sturdivant RX. Applied Logistic Regression. 3rd ed. 
27. Eppler S, Gordon MS, Redfern CH, et al. Lack of a pharmacokinetic interaction between trastuzumab and carboplatin in the presence of docetaxel: results from a phase Ib study in patients with HER2-positive metastatic or locally advanced inoperable solid tumors. Anticancer Drugs. 2015;26(4):448-455.

28. Hoffler D, Koeppe P, Paeske B. Pharmacokinetics of azithromycin in normal and impaired renal function. Infection. 1995;23(6):356-361.

29. Cocco G, Jerie P. Torsades de pointes induced by the concomitant use of ivabradine and azithromycin: an unexpected dangerous interaction. Cardiovasc Toxicol. 2015;15(1):104-106.

30. Pearson EC, Woosley RL. QT prolongation and torsades de pointes among methadone users: reports to the FDA spontaneous reporting system. Pharmacoepidemiology and drug safety. 2005;14(11):747-753.

31. Hancox JC, Hasnain M, Vieweg WV, Gysel M, Methot M, Baranchuk A. Erythromycin, QTc interval prolongation, and torsade de pointes: Case reports, major risk factors and illness severity. Ther Adv Infect Dis. 2014;2(2):47-59.

32. Roe CM, Odell KW, Henderson RR. Concomitant use of antipsychotics and drugs that may prolong the QT interval. J Clin Psychopharmacol. 2003;23(2):197-200.

33. Woosley RL, Romero KA. QTdrugs List. www.Crediblemeds.org. Accessed March 8, 2017.

34. Kestenbaum B, Rudser KD, Shlipak MG, et al. Kidney function, electrocardiographic findings, and cardiovascular events among older adults. Clin J Am Soc Nephrol. 2007;2(3):501-508.

35. Patane S. Torsade de pointes, QT interval prolongation and renal disease. Int J Cardiol. 2011;149(2):241-242. 
Table 2.1 Demographics and clinical characteristics of cases and controls

\begin{tabular}{|l|c|c|c|c|}
\hline \multirow{2}{*}{ Characteristics } & \multicolumn{2}{|c|}{$\begin{array}{c}\text { Cases } \\
\text { (n=4,612) }\end{array}$} & \multicolumn{2}{c|}{$\begin{array}{c}\text { Controls } \\
\text { (n= 18,448) }\end{array}$} \\
\hline & Mean & SD & Mean & SD \\
\hline Age, y & 59.5 & 13.7 & 59.5 & 13.7 \\
\hline Charlson comorbidity index & 2.8 & 3.1 & 0.5 & 1.3 \\
\hline & & & & \\
\hline Charlson comorbidity index & No. & \% & No. & \% \\
\hline 0 & 1259 & 27.3 & 14537 & 78.8 \\
\hline 1 & 876 & 19.0 & 1769 & 9.6 \\
\hline 2 & 591 & 12.8 & 939 & 5.1 \\
\hline$\geq 3$ & 1886 & 40.9 & 1203 & 6.5 \\
\hline & & & & \\
\hline Comorbidities & No. & $\mathbf{\%}$ & No. & \% \\
\hline AIDS/HIV & 27 & 0.6 & 19 & 0.1 \\
\hline Angina pectoris & 207 & 4.5 & 161 & 0.9 \\
\hline Any malignancy & 745 & 16.2 & 782 & 4.2 \\
\hline Cardiac arrhythmia & 1846 & 40.0 & 974 & 5.3 \\
\hline Cardiomyopathy & 835 & 18.1 & 185 & 1.0 \\
\hline Cerebrovascular disease & 647 & 14.0 & 571 & 3.1 \\
\hline Chronic kidney disease & 849 & 18.4 & 449 & 2.4 \\
\hline Chronic pulmonary disease & 1349 & 29.3 & 1244 & 6.7 \\
\hline Congestive heart failure & 1456 & 31.6 & 464 & 2.5 \\
\hline Coronary artery disease & 1450 & 31.4 & 1063 & 5.8 \\
\hline Dementia & 64 & 1.4 & 90 & 0.5 \\
\hline $\begin{array}{l}\text { Diabetes with chronic } \\
\text { complication }\end{array}$ & 537 & 11.6 & 371 & 2.0 \\
\hline $\begin{array}{l}\text { Diabetes without chronic } \\
\text { complication }\end{array}$ & 1508 & 32.7 & 1554 & 8.4 \\
\hline Heart murmurs & 133 & 2.9 & 118 & 0.6 \\
\hline Heart valve disease & 1001 & 21.7 & 605 & 3.3 \\
\hline Hemiplegia or paraplegia & 80 & 1.7 & 39 & 0.2 \\
\hline Hypokalemia & 343 & 7.4 & 157 & 0.9 \\
\hline Metastatic solid tumor & 240 & 5.2 & 81 & 0.4 \\
\hline Mild liver disease & 361 & 7.8 & 267 & 1.4 \\
\hline Moderate or severe liver disease & 47 & 1.0 & 22 & 0.1 \\
\hline Myocardial infarction & 475 & 10.3 & 197 & 1.1 \\
\hline Palpitation & 438 & 9.5 & 320 & 1.7 \\
\hline Peptic ulcer disease & 85 & 1.8 & 70 & 0.4 \\
\hline Peripheral vascular disease & 688 & 14.9 & 540 & 2.9 \\
\hline Renal disease & 799 & 17.3 & 399 & 2.2 \\
\hline Rheumatic disease & 181 & 3.9 & 205 & 1.1 \\
\hline Ventricular septal defect & 0 & 0.0 & 1 & 0.0 \\
\hline & & & & \\
\hline & & & & \\
\hline & & & & \\
\hline
\end{tabular}




\begin{tabular}{|l|c|c|c|c|}
\hline $\begin{array}{l}\text { Concomitant QT medication } \\
\text { exposure }\end{array}$ & No. & $\mathbf{\%}$ & No. & \% \\
\hline $\begin{array}{l}\text { Concomitant QT antibiotic and } \\
\text { other QT medication 30 days } \\
\text { prior to index date }\end{array}$ & 20 & 0.4 & 17 & 0.1 \\
\hline $\begin{array}{l}\text { Proximal QT antibiotic and other } \\
\text { QT medication 30 days prior to } \\
\text { index date }\end{array}$ & 46 & 1.0 & 35 & 0.2 \\
\hline $\begin{array}{l}\text { Number of QT medication use 10 } \\
\text { days prior to index date }\end{array}$ & & & & \\
\hline 0 & 3310 & 71.8 & 17151 & 93.0 \\
\hline 1 & 879 & 19.1 & 928 & 5.0 \\
\hline 2 & 107 & 2.3 & 66 & 1.6 \\
\hline$\geq 3$ & & 6.8 & 306 & 0.4 \\
\hline
\end{tabular}


Table 2.2 Concomitant QT drug use and predictors of ventricular arrhythmia or sudden death

\begin{tabular}{|l|c|c|}
\hline Study variable & $\begin{array}{c}\text { Adjusted odds } \\
\text { ratio }\end{array}$ & $\begin{array}{c}\mathbf{9 5 \%} \\
\text { confidence } \\
\text { interval }\end{array}$ \\
\hline $\begin{array}{l}\text { Concomitant QT-prolonging medication use } \\
\text { (QT antibiotic + other QT medication) }\end{array}$ & 2.91 & $1.19-7.08$ \\
\hline Charlson comorbidity index & & \\
\hline 0 & 1.00 & N/A \\
\hline 1 & 3.40 & $2.95-3.92$ \\
\hline 2 & 3.81 & $3.20-4.52$ \\
\hline$\geq 3$ & 4.67 & $3.82-5.72$ \\
\hline Comorbidities & & \\
\hline Cardiac arrhythmia & 3.58 & $3.11-4.11$ \\
\hline Cardiomyopathy & 2.01 & $1.54-2.62$ \\
\hline Chronic pulmonary disease & 1.40 & $1.22-1.60$ \\
\hline Congestive heart failure & 1.83 & $1.50-2.24$ \\
\hline Coronary artery disease & 1.86 & $1.61-2.15$ \\
\hline Diabetes without chronic complication & 1.26 & $1.10-1.44$ \\
\hline Heart murmurs & 1.48 & $1.03-2.12$ \\
\hline Heart valve disease & 1.48 & $1.26-1.74$ \\
\hline Hypokalemia & 2.11 & $1.62-2.74$ \\
\hline Metastatic solid tumor & 3.25 & $2.31-4.58$ \\
\hline Mild liver disease & 1.39 & $1.11-1.74$ \\
\hline Myocardial infarction & 1.36 & $1.08-1.76$ \\
\hline Palpitation & 1.74 & $1.40-2.15$ \\
\hline
\end{tabular}


Table 2.3 Proximal QT drug use ( \pm 15 days) and predictors of ventricular arrhythmia or sudden death

\begin{tabular}{|l|c|c|}
\hline Study variable & $\begin{array}{c}\text { Adjusted odds } \\
\text { ratio }\end{array}$ & $\begin{array}{c}\mathbf{9 5 \%} \\
\text { confidence } \\
\text { interval }\end{array}$ \\
\hline $\begin{array}{l}\text { Proximal QT-prolonging medication use (QT } \\
\text { antibiotic + other QT medication)* }\end{array}$ & 2.2 & $1.23-3.40$ \\
\hline Charlson comorbidity index & & \\
\hline 0 & 1.00 & N/A \\
\hline 1 & 3.40 & $2.95-3.92$ \\
\hline 2 & 3.50 & $3.20-4.52$ \\
\hline$\geq 3$ & 4.70 & $3.84-5.74$ \\
\hline Comorbidities & & \\
\hline Cardiac arrhythmia & 3.56 & $3.10-4.09$ \\
\hline Cardiomyopathy & 2.00 & $1.53-2.61$ \\
\hline Chronic kidney disease & 1.66 & $1.37-2.00$ \\
\hline Chronic pulmonary disease & 1.40 & $1.23-1.60$ \\
\hline Congestive heart failure & 1.83 & $1.50-2.24$ \\
\hline Coronary Artery Disease & 1.86 & $1.61-2.15$ \\
\hline Diabetes without chronic complication & 1.25 & $1.09-1.43$ \\
\hline Heart murmurs & 1.50 & $1.04-2.15$ \\
\hline Heart valve disease (cortic stenosis) & 1.47 & $1.25-1.73$ \\
\hline Hypokalemia & 2.11 & $1.63-2.75$ \\
\hline Metastatic solid tumor & 3.23 & $2.30-4.54$ \\
\hline Mild liver disease & 1.40 & $1.12-1.75$ \\
\hline Myocardial infarction & 1.39 & $1.09-1.78$ \\
\hline Palpitation & 1.74 & $1.41-2.16$ \\
\hline
\end{tabular}

$* \pm 15$ days allowed between durations of prescriptions 
Table 2.4 Number of QT-prolonging medications and predictors of ventricular arrhythmia or sudden death

\begin{tabular}{|l|c|c|}
\hline Study variable & $\begin{array}{c}\text { Adjusted odds } \\
\text { ratio }\end{array}$ & $\begin{array}{c}\text { 95\% confidence } \\
\text { interval }\end{array}$ \\
\hline Number of QT-prolonging drug used & 1.00 & N/A \\
\hline 0 & 2.62 & $2.28-3.01$ \\
\hline 1 & 2.57 & $2.05-3.22$ \\
\hline 2 & 3.11 & $2.05-4.71$ \\
\hline$\geq 3$ & & N/A \\
\hline Charlson comorbidity index & 1.00 & $2.69-3.60$ \\
\hline 0 & 3.11 & $2.91-4.14$ \\
\hline 1 & 3.47 & $3.35-5.06$ \\
\hline 2 & 4.11 & $3.11-4.11$ \\
\hline$\geq 3$ & & $1.54-2.62$ \\
\hline Comorbidities & 3.58 & $1.22-1.60$ \\
\hline Cardiac arrhythmia & 2.01 & $1.50-2.24$ \\
\hline Cardiomyopathy & 1.40 & $1.61-2.15$ \\
\hline Chronic pulmonary disease & 1.83 & $1.10-1.44$ \\
\hline Congestive heart failure & 1.86 & $1.03-2.12$ \\
\hline Coronary artery disease & 1.26 & $1.26-1.74$ \\
\hline Diabetes without chronic complication & 1.48 & $1.62-2.74$ \\
\hline Heart murmurs & 1.48 & $2.31-4.58$ \\
\hline Heart valve disease & 2.11 & $1.11-1.74$ \\
\hline Hypokalemia & 3.25 & $1.08-1.76$ \\
\hline Metastatic solid tumor & 1.39 & $1.40-2.15$ \\
\hline Mild liver disease & 1.36 & \\
\hline Myocardial infarction & 1.74 & \\
\hline Palpitation & & \\
\hline
\end{tabular}


Manuscript III. Risk of drug-induced ventricular arrhythmia and sudden death with QT-prolonging antibiotics

Yizhou Ye, M.S. ${ }^{1}$, Kerry L. LaPlante, Pharm.D. ${ }^{1,2}$, E P. Larrat, Ph.D. ${ }^{1}$, and Aisling R. Caffrey, Ph.D., M.S. ${ }^{1,2,3}$

1. College of Pharmacy, Department of Pharmacy Practice, University of Rhode Island, Kingston, RI, United States

2. Veterans Affairs Medical Center, Infectious Diseases Research Program and Center of Innovation in Long Term Services and Supports, Providence, RI, United States

3. School of Public Health, Brown University, Providence, RI, United States

Corresponding Author: Aisling R. Caffrey, PhD, MS, Assistant Professor, University of Rhode Island; 7 Greenhouse Road, Kingston, RI 02881; office: (401) 874-5320; e-mail: Aisling_Caffrey@uri.edu

Abstract word count: 263

Manuscript word count: 3670

Table/figure count: $1 / 5$

Funding: Unfunded

Keywords: Azithromycin, drug safety, fluoroquinolone, macrolide, QT prolongation, sudden death, ventricular arrhythmia

Target journal: Clinical Infectious Diseases

Publication status: Preparing for publication 


\section{ABSTRACT}

Background: Warnings regarding the increased risk of ventricular arrhythmia with azithromycin have been issued by regulatory agencies. However, conflicting results have been observed in retrospective studies evaluating this association.

Objectives: To evaluate the 10-day and 30-day risk of ventricular arrhythmia or sudden death associated with azithromycin relative to amoxicillin in a national, commercially insured population.

Methods: A retrospective cohort study was conducted using an administrative health claims database from a large national insurer. The study cohort consisted of patients who filled an outpatient prescription between 2011 and 2013 for macrolide and fluoroquinolone antibiotics, or amoxicillin. Amoxicillin episodes were 1-to-1 matched to macrolide and fluoroquinolone episodes on propensity scores. Inpatient admissions or emergency department visits with a primary diagnosis of ventricular arrhythmia were assessed within 10 days and 30 days of prescription dispensing using Cox proportional hazards models.

Results: The use of azithromycin was not associated with an elevated 10-day (hazard ratio [HR]: 1.44, 95\% confidence interval [CI]: 0.79-2.64, $\mathrm{p}=0.23)$ or 30-day (HR: 1.17, 95\% CI: 0.81-1.68, $\mathrm{p}=0.41$ ) risk of ventricular arrhythmia or sudden death compared with amoxicillin. A class risk for macrolides or fluoroquinolones was also not observed. In a sensitivity analysis which expanded the outcome definition to include primary and secondary diagnoses, macrolides, as a class, had a 68\% higher 10- 
day (HR: 1.68, 95\% CI: 1.16-2.44, p=0.03) risk of ventricular arrhythmia or sudden death.

Conclusions: Among a commercially insured population, azithromycin use was not associated with an increased risk of ventricular arrhythmia or sudden death compared with amoxicillin. Our findings suggest that the cardiac risk associated with azithromycin may be population specific. 


\section{BACKGROUND}

Macrolide and fluoroquinolone antibiotics are known to increase the risk of cardiac arrhythmia, including QT prolongation and torsades de pointes (TdP). ${ }^{1,2}$

Several observational epidemiologic studies suggest that azithromycin may also have an increased risk of ventricular arrhythmia and sudden death. ${ }^{3-5}$ One retrospective cohort study published in 2012 reported a higher risk of cardiovascular death associated with azithromycin compared with no antibiotic use (hazard ratio [HR]: 2.88, 95\% confidence interval [CI]: 1.79-4.63) or amoxicillin (HR: 2.49, 95\% CI: 1.38-4.50) in a Medicaid population. ${ }^{4}$ One year later, the United States Food and Drug Administration (FDA) issued a warning to urge healthcare professionals to consider potential cardiovascular risks when prescribing azithromycin. ${ }^{6}$ Subsequent observational studies reported similar increased risks of serious arrhythmia, cardiovascular death, and all-cause mortality among patients taking azithromycin among Veterans Affairs patients and in the general population of Taiwan.,

Meanwhile, several observational studies observed a relatively similar cardiac toxicity profile with azithromycin, as compared with no antibiotic use or other antibiotics. $^{7-9}$ In the Danish adult population, azithromycin use $(n=1,102,050)$ was not associated with an increased risk of cardiovascular death compared to either no antibiotic use (rate ratio $=0.93,95 \%$ CI $0.56-1.55$ ) or penicillin- $\mathrm{V}$ use (adjusted absolute risk different $=-1,95 \%$ CI -9 to +11$).{ }^{8}$ Another study among the general population of Ontario, Canada found that macrolide antibiotic use was not associated with a higher risk of ventricular arrhythmia (relative risk [RR]: 1.06, 95\% CI: 0.831.36) compared to non-macrolide antibiotics. ${ }^{7}$ The same study also observed a lower 
risk of all-cause mortality for azithromycin (RR: 0.82, 95\% CI: 0.78-0.86) compared to non-macrolide antibiotics.

Motivated by the FDA warnings and conflicting results observed in different populations worldwide, we sought to assess the risk of ventricular arrhythmia (VA) or sudden death (SD) for macrolide and fluoroquinolone antibiotics in database national commercially insured population in the United States (US) and compare the results with previously published observational studies. 


\section{METHODS}

\section{Data source}

We utilized the de-identified Optum Clinformatics ${ }^{\mathrm{TM}}$ DataMart database (OptumInsight, Eden Prairie, MN) for this study, which is an administrative health claims database from a large national insurer. We utilized member eligibility files, medical claims, confinement claims, and pharmacy claims to conduct this study.

\section{Study population}

We identified outpatient prescription of oral macrolide antibiotics (azithromycin, clarithromycin, and erythromycin), fluoroquinolone antibiotics (ciprofloxacin, gemifloxacin, levofloxacin, moxifloxacin, and ofloxacin), or amoxicillin (including amoxicillin-calvulanate) from January 1, 2011 to November 30, 2013. The prescription fill date was defined as the index date $\left(\mathrm{t}_{0}\right)$ of each antibiotic episode. Subsequent antibiotic episodes for each unique patient were only included when the subsequent index date was more than 30 days after the end date (index date + days of supply) of a previous episode. Eligible antibiotic episodes were selected using the same criteria as previous studies. ${ }^{4}$ The inclusion criteria were (1) at least 1 year of continuous enrollment prior to the index date for the assessment of baseline characteristics including medical history; and (2) at least 18 years of age on the index date. We excluded those that were (1) missing age or gender information; (2) hospitalized 30 days prior to the index date; (3) treated with multiple study antibiotics on the index date; (4) nursing home or other long-term care facility resident; and (5) previously diagnosed with cancer, organ transplant, congenital cardiovascular 
anomalies, other congenital anomalies/childhood conditions, substance abuse, human immunodeficiency virus (HIV) infection, or life-threatening end-stage illness one year prior to the index date (Appendix B). ${ }^{4}$

\section{Drug exposure}

Eligible antibiotic episodes were categorized into three treatment groups and one control group based on filled outpatient oral antibiotics: (1) azithromycin; (2) all macrolide antibiotics, which included azithromycin, clarithromycin, and erythromycin; (3) all fluoroquinolones, which included ciprofloxacin, gemifloxacin, levofloxacin, moxifloxacin, and ofloxacin; and (4) amoxicillin serving as the active comparator group or control group for each of the three aforementioned treatment groups, which included amoxicillin alone and amoxicillin-clavulanate. Previous research has shown that amoxicillin use does not increase the risk of QT prolongation related cardiovascular outcomes. ${ }^{4,10}$

\section{Outcome definitions}

The study outcome was ventricular arrhythmia and sudden death (referred to as an “event” for the rest of the manuscript), defined as having a primary diagnosis during a hospitalization or first-listed emergency department (ED) visit for severe ventricular arrhythmia, cardiac arrest, sudden death, or instantaneous death (International Classification of Diseases, Ninth Revision, Clinical Modification [ICD9-CM] codes: 427.1, 427.4, 427.41, 427.42, 427.5, 798, 798.1, and 798.2). This outcome definition was validated in previous studies with positive predictive values

(PPVs) of over $80 \% .{ }^{11-14}$ Drug-induced QT prolongation is dose-related and the effect 
diminishes quickly as study medications are metabolized and excreted. ${ }^{15}$ Therefore, we conducted the analysis on two study endpoints which were day 10 and day 30 after the index date. ${ }^{3,4,7}$

\section{Statistical Analysis}

Descriptive analyses were performed for the study cohort. The characteristics of the study population, including age, gender, year, month, and geographical region were assessed at the index date. Diagnoses of conditions related to indications were available in the data source and were controlled for in the propensity score. Medical history, medication use, and healthcare utilization were assessed from inpatient and outpatient diagnoses and procedures in the medical and confinement files, as well as from prescription information in the pharmacy files during the 1-year period before the index date (Appendix C). ${ }^{4}$

In this study, propensity score matching was used to balance the baseline characteristics of the study groups for the purpose of estimating the probability of exposure. ${ }^{16}$ Propensity score methods allow one to minimize the effects of observed confounding when using observational data. For each comparison (azithromycinamoxicillin, macrolides-amoxicillin, fluoroquinolone-amoxicillin), a separate propensity score was calculated using logistic regression. The propensity score model was developed through an iterative, manual backward elimination approach using Wald statistics and likelihood ratio tests ( $\mathrm{p}$-value $<0.2$ for main effects). ${ }^{17,18}$ Parson's greedy matching was used to match within 0.000001 of the propensity score, without replacement, one amoxicillin episode from the control group to a single episode in 
each of the three treatment groups. The adequacy of matching was assessed with a visual assessment of the distribution of propensity scores and by calculating the standardized difference between study groups. ${ }^{17}$ Differences of less than $10 \%$ were considered negligible between exposure groups. ${ }^{17}$ If important residual systematic differences were identified, interactions terms between covariates already in the model would be assessed. ${ }^{19}$

In each matched comparison cohort, antibiotic episodes were followed for a minimum of 10 days and up to 30 days after the index date. Date of diagnosis of the outcome was used to define time to event. The Cox proportional hazards ( $\mathrm{PH}$ ) model was used to estimate the relative hazard of ventricular arrhythmia and sudden death by regressing survival on treatment status. ${ }^{16,20}$ The PH assumption was evaluated from the statistical significance of the correlation between the Schoenfeld residuals and the ranking of individual failure times. ${ }^{20}$ Since antibiotic episodes selected in matched cohorts were more likely to be similar to each other than randomly selected subjects, study outcomes were considered dependent within matched pairs. Therefore, the robust variance estimator was used to account for the matched nature of the study cohort. Estimated HR with 95\% confidence intervals (CIs) for each comparison was obtained.

For sensitivity analyses, we expanded the definition of the event from a primary diagnosis to any diagnoses within a hospitalization or ED visit. This definition has lower PPVs than the primary definition, however the PPVs for this definition are still over 70\%. ${ }^{13}$ We also conducted a subgroup analysis among patients with a history of chronic kidney disease (CKD) because they have a higher risk of 
developing QT prolongation. Studies have shown that patients with chronic kidney disease had longer QTc than patient without CKD. ${ }^{21,22}$ Furthermore, patients with CKD had impaired renal excretion of azithromycin, thereby leading to higher intravascular concentration of the drug. Because the pro-arrythmic effect of macrolides is dose-dependent, the increased concentration of the drug in patients with CKD may increase their risk of ventricular arrhythmia and sudden death. ${ }^{15,21,23}$

All statistical analyses were performed using SAS Enterprise Guide, version 7.1 (SAS Institute Inc., Cary, NC, USA). This study was reviewed and approved as exempt by the University of Rhode Island's Institutional Review Board. 


\section{RESULTS}

\section{Characteristic of the study cohorts}

The study population included 15,634,507 enrollees who filled a total of 12,774,368 outpatient prescriptions for oral macrolide antibiotics (azithromycin, clarithromycin, and erythromycin), fluoroquinolone antibiotics (ciprofloxacin, gemifloxacin, levofloxacin, moxifloxacin, ofloxacin), and amoxicillin (including amoxicillin-calvulanate) during the study period. After applying the exclusion criteria, we identified 5,105,097 eligible prescriptions episodes: 28\% azithromycin, 3\% other macrolides (clarithromycin and erythromycin), 25\% fluoroquinolones, and 43\% amoxicillin (Table 3.1). The mean age of the entire study population was 45 years (range: 18-87) and 61\% were female.

Azithromycin, all macrolide and all fluoroquinolone episodes were matched 1:1 to amoxicillin episodes on propensity scores. An equal number of amoxicillin prescription episodes were matched to azithromycin, all macrolides, and all fluoroquinolones. The azithromycin-amoxicillin cohort consisted of 1,329,575 azithromycin prescription episodes with an equal number of amoxicillin prescriptions episodes. The fluoroquinolones-amoxicillin and macrolide-amoxicillin cohorts consisted of 1,096,138 and 1,457,873 prescription episodes of fluoroquinolone and macrolide prescription episodes, each with an equal number of amoxicillin prescriptions episodes (Figure 3.1).

Propensity score matching resulted in similar propensity score distributions between the treatment and control groups (Appendix D). The propensity score also 
successfully balanced the study variables that contributed to the propensity score for each model, as measured by standardized differences (Appendix D). Standardized differences suggested insignificant differences between the treatment and control groups. Therefore, interaction terms between covariates were not assessed.

\section{Ventricular arrhythmia or sudden death}

\section{Azithromycin-amoxicillin}

We identified 1,329,575 azithromycin prescription episodes (mean age: 43 years, females: 61\%) and an equal number of propensity matched amoxicillin episodes. Among azithromycin recipients, there were 26 events (20 per 1 million episodes) during the 10-day follow-up period. During the same 10-day follow-up

period, 18 events (13 per 1 million episodes) occurred among amoxicillin recipients. During the remaining 11-30 days of follow-up, 37 and 36 additional events occurred among azithromycin and amoxicillin recipients, respectively. The cumulative incidence was 47 and 41 per 1 million episodes for azithromycin and amoxicillin recipients, respectively. In time-to-event analyses, azithromycin was not associated with ventricular arrhythmia or sudden death, compared with amoxicillin (Figure 3.2). In the 10-day analysis, risk of ventricular arrhythmia or sudden death was elevated with azithromycin but not statistically significant (HR: 1.44, 95\% CI: 0.79-2.64, $\mathrm{p}=0.230$ ). In the longer follow-up period of 30 days, the risk remained non-significant for azithromycin with a lower HR of 1.17 (95\% CI: 0.81-1.68, p=0.407).

\section{All macrolides-amoxicillin}


We identified 1,457,873 macrolide prescription episodes (mean age: 43 years, females: 61\%) and an equal number of propensity matched amoxicillin episodes. There were 26 and 23 events during the first 10 days after dispensing macrolides and amoxicillin, respectively, with an incidence of 18 and 16 per 1 million episodes, respectively. During the 30-day follow-up period, 63 and 59 events occurred among all macrolides and amoxicillin recipients, respectively. Macrolide antibiotics were not associated with ventricular arrhythmia or sudden death in the 10-day (HR: 1.13, 95\% CI: 0.64-1.98, p=0.669) and 30-day follow-up periods (HR: 1.07, 95\% CI 0.75-1.52, $\mathrm{p}=0.717$; Figure 3.3).

\section{All fluoroquinolones-amoxicillin}

We identified 1,096,138 fluoroquinolone prescription episodes (mean age: 46 years, females: 63\%) and an equal number of propensity matched amoxicillin episodes for this cohort. Among fluoroquinolone recipients, there were 24 events (22 per 1 million episodes) during the 10-day follow-up period. During the same follow-up period, 22 events (20 per 1 million episodes) occurred among amoxicillin recipients. During the 30-day follow-up period, 64 and 59 events occurred among fluoroquinolone and amoxicillin recipients, respectively with a cumulative incidence of 58 and 54 per 1 million episodes for fluoroquinolone and amoxicillin recipients, respectively. Fluoroquinolones were not associated with ventricular arrhythmia or sudden death (Figure 3.4). In the 10-day follow-up period, the HR was 1.09 (95\% CI: 0.61-1.94, $\mathrm{p}=0.768$ ), which decreased to 0.92 at 30-days (95\% CI: 0.65-1.31, $\mathrm{p}=0.922)$. 


\section{Sensitivity Analysis}

Sensitivity analyses showed similar results when we expanded the event definition from a primary diagnosis only to any recorded diagnoses during hospital stays or ED visits. Azithromycin and fluoroquinolones were not associated with a higher risk of ventricular arrhythmia or sudden death compared with amoxicillin (Appendix E). The sensitivity analysis for macrolides also showed no increased risk in the 30-day follow-up period (HR: 1.24, 95\% CI 0.97-1.58, $\mathrm{p}=0.950$ ) but did demonstrate a significant increased risk of ventricular arrhythmia or sudden death at 10 days (HR: 1.68, 95\% CI 1.16-2.44, $\mathrm{p}=0.031$, Figure 3.5).

In the subgroup analysis among patients with chronic kidney disease, risk of ventricular arrhythmia or sudden death was similar in all comparison groups (10-day HR 1.65, 95\% CI 0.39-6.90, p=0.493; 30-day HR 1.16, 95\% CI 0.39-3.44, p=0.796; results not presented in tables or figures). 


\section{DISCUSSION}

In our study, we did not observe a significantly increased risk of ventricular arrhythmia or sudden death associated with azithromycin use alone, however when evaluating all macrolides, a 68\% higher risk at 10 days was identified in our analysis of the event as either a primary or secondary diagnosis. To our knowledge, this is the first observational study to assess the association of ventricular arrhythmia and sudden death with azithromycin, all macrolides, and all fluoroquinolones in a large, national, commercially insured population. Our inclusion criteria were similar to those used in previous studies, ${ }^{3,4}$ and we evaluated the risk of cardiac adverse events at multiple time points (10 days and 30 days) so our results could be compared with these other studies. ${ }^{5,7}$ Furthermore, our study outcome has been validated in several populations. $^{11,13,14}$

It was previously thought that azithromycin did not exhibit a pro-arrhythmic potential as seen with the other macrolides, erythromycin and clarithromycin. ${ }^{24}$ Based on in vitro preclinical studies, azithromycin was observed to be weak cardiac human ether-a-go-go-related gene (hERG) potassium channel blocker, and it is widely recognized that pharmacological inhibition hERG potassium channel is correlated with drug-induced QT-prolongation. ${ }^{24-27}$ Azithromycin is also a group 3 cytochrome P-450 CYP3A4 inhibitor which binds weakly with CYP3A4, and thus has less potential for adverse effects and drug-drug interactions with other CYP3A4 inhibitors such as erythromycin. ${ }^{10,28-30}$ However, we have observed a great discrepancy between in vitro studies and real-world clinical practice that is now largely recognized as the result of genetic factors. $^{31}$ 
A previous population-based study in senior patients (age > 65 years) from Ontario, Canada reported no association between macrolide use and ventricular arrhythmia compared to non-macrolide antibiotic use in the 14 and 30 days after the index date. ${ }^{7}$ As compared with the Canadian study, we also assessed all macrolide antibiotics together and did not observe an increased risk at 30 days. However, our findings differed from the Canadian study at the shorter follow-up time point, where we observed a significant association with macrolides. Both studies assessed macrolides as the exposure of interest but different comparators were used. In our study, we selected amoxicillin as the comparator due to its low cardiovascular risk. ${ }^{4,10}$ The comparator used in the Canadian study was defined as non-macrolide antibiotics, which included amoxicillin, cefuroxime, or levofloxacin. Levofloxacin is a fluoroquinolone antibiotic has been associated QT-prolonging effect. ${ }^{32,33}$ The inclusion of levofloxacin in the non-macrolide group may have diminished the risk difference between the study groups. Study outcomes were slightly different as well; we included both ventricular arrhythmia and sudden death, while the Canadian study only assessed ventricular arrhythmia.

Our findings related to the risk of azithromycin and cardiac adverse events were in agreement with a population-based study from Denmark. In the Danish general population, there was no association between azithromycin use and the risk of cardiovascular death compared to either penicillin V (5-day rate ratio [RR] 0.93, 95\% CI, 0.56-1.55) or no antibiotics (5-day RR 2.85, 95\% CI, 1.13-7.24) in 5, 10, and 35 day follow-up periods. ${ }^{8}$ Like our study population, the Danish general population was also younger (mean age for azithromycin use: 39.7 years) and healthier. ${ }^{8}$ Our study 
varied from the Danish study, in that we used different comparators and a different definition of the study outcome.

Alternatively, a study in the Tennessee Medicaid population observed a higher risk of cardiovascular death associated with azithromycin than with either no antibiotic use or amoxicillin use. ${ }^{4}$ Factors that may contribute to the conflicting study results between our study and the Tennessee Medicaid study include the different demographic and baseline characteristics of the study populations. Risk factors for drug-induced ventricular arrhythmia and sudden death include female gender, ${ }^{15,34}$ older age,${ }^{15,34}$ history of chronic kidney disease, ${ }^{21,35}$ hypokalemia, ${ }^{36,37}$ existing cardiac conditions, ${ }^{25}$ and use of other QT-prolonging medications. ${ }^{25,38}$ Unlike the Tennessee Medicaid study, our study was conducted in a younger, privately-insured population. Our propensity score matched cohorts were younger and healthier overall, with fewer comorbidities. ${ }^{4}$ In our study, the mean age of the azithromycin-amoxicillin matched cohort was 43 years, whereas that of the Tennessee Medicaid study was 48 years. In our azithromycin-amoxicillin cohort, only 2.0\% had complications of diabetes, $0.9 \%$ had heart failure, 9.7\% used angiotensin-converting-enzyme (ACE) inhibitors, 8.4\% used beta-blockers, and 7.4\% had inpatient stays. In the Tennessee Medicaid study, $7.5 \%$ of their matched cohort had complications of diabetes, $4.3 \%$ had heart failure, 28.1\% used ACE inhibitors, 21.5\% used beta-blockers, and 23.0\% had inpatient stays. Our study also differed in the definition of the study outcome and prevalence of that outcome, as they assessed a broader definition of death, as cardiovascular rather than sudden death, and they did not assess rates of ventricular arrhythmia. In our study, the 10-day cumulative incidence of ventricular arrhythmia and sudden death was 20 per 
million azithromycin episodes. In contrast, the Tennessee Medicaid study reported the 10-day cumulative incidence of cardiovascular death to be 360 per million azithromycin episodes.

An increased risk of cardiac arrhythmias was also reported with azithromycin among Veterans in the US and in Taiwan. The VA study reported an increased 5-day risk of serious cardiac arrhythmias (HR 2.13, 95\% CI 1.44-3.15) associated with azithromycin. ${ }^{3}$ Despite the same drug exposure group, there was a considerable age difference between the older VA population and ours (mean age 56 vs. 43 years). The VA study only assessed the outcome of ventricular arrhythmia, with a 10-day cumulative incidence rate of 200 per million azithromycin episodes. As mentioned above, the 10-day cumulative incidence of ventricular arrhythmia and sudden death was only 20 per million azithromycin episodes in our study. Similarly, azithromycin was associated with an increased 7-day risk of ventricular arrhythmia and sudden death (adjusted odds ratio [aOR] 4.32, 95\% CI, 2.95-6.33) and cardiovascular death (aOR 2.62, 95\% CI, 1.69-4.06) in the general population of Taiwan. ${ }^{5}$ Though our study population differed from the Taiwanese study population in many aspects, such as race/ethnicity and healthcare practice, azithromycin users were similar in several baseline characteristics, including age (45 vs. 43 years), and other comorbidities, including congestive heart failure (1.5\% vs. 1.0\%). Like our study, the Taiwanese study also used amoxicillin as the comparator. Nonetheless, the 7-day cumulative incidence rate was 520 per million azithromycin episodes in the Taiwanese general population since they used a broader definition of ventricular arrhythmia and sudden death from both inpatient and outpatient visits. This outcome definition which 
included outpatient visits for ventricular arrhythmia and sudden death has not been validated and resulted in a high observed cumulative incidence in the general population of Taiwan.

Several previous studies also observed diminishing risks during longer followup periods. During the shorter follow-up period of 5 days, both the Tennessee Medicaid and VA studies reported an increased risk associated with azithromycin use as compared with amoxicillin use. During days 6 to 10 of follow-up, the risk was no longer significant in either the Tennessee Medicaid study (HR 0.88, 95\% CI 0.431.80) or the VA study (HR 0.73, 95\% CI 0.49-1.10). ${ }^{3,4}$ For studies which assessed 30day risk, none reported an increased risk. ${ }^{7,8}$

Our study has several important limitations. In lieu of actual drug administration information, we relied on dispensed prescriptions records from an administrative claims database as our measure of exposure. Also, utilization of erythromycin and clarithromycin was uncommon in our population, therefore risk could not be assessed for individual macrolide antibiotics other than azithromycin. In addition, though diagnoses of infection were available in the data source and were controlled for in the propensity score, specific indications for each dispensed antibiotic were not available in the pharmacy claims data. In order to minimize the potential influence of confounding by the indication, we used amoxicillin, another antibiotic that has similar indications and minimal cardiac toxicity. ${ }^{4}$ Several risk factors for ventricular arrhythmia and sudden death, such as smoking status and body mass index were not available in our data source. ${ }^{15}$ Therefore, despite propensity score matching using predicted probabilities from a model which included many baseline covariates, 
there was still potential for unmeasured confounding in our study. ${ }^{39}$ Another limitation was that our study could not assess a more proximal time frame to the prescription dispensing. Azithromycin is commonly prescribed for 5 days but we could only assess the study outcome at 10 days due to the low cumulative incidence observed in our study population. Finally, due to the nature of using an administrative claims database, we relied on diagnosis codes of ventricular arrhythmia and sudden death. Therefore, had these events occurred but not resulted in claims with these specific diagnoses, misclassification could have occurred. It is difficult to measure the true prevalence of QT prolongation as there is no method to quantify undetected events. ${ }^{40,41}$ Without the true denominator, validated algorithms for QT prolongation identification report only PPV but not sensitivity/specificity for the same reason. ${ }^{11,42}$ We did attempt to use the most accurate definition possible, by using two methods with high PPVs for identifying the study outcome. Nevertheless, bias towards the null for all studies utilizing these definitions for identifying events related to QT prolongation is a possibility as we would not expect this misclassification to differ by exposure status. 


\section{CONCLUSION}

Among this privately-insured adult population, azithromycin and fluoroquinolones were not associated with a higher risk of ventricular arrhythmia and sudden death compared with amoxicillin. Macrolide antibiotics demonstrated a higher 10-day risk of ventricular arrhythmia and sudden death compared with amoxicillin, suggesting a class effect, with differing quantitative risk between azithromycin and the other macrolides, clarithromycin and erythromycin. 


\section{REFERENCES}

1. Owens RC, Jr., Nolin TD. Antimicrobial-associated QT interval prolongation: pointes of interest. Clinical infectious diseases : an official publication of the Infectious Diseases Society of America. 2006;43(12):1603-1611.

2. Kim MH, Berkowitz C, Trohman RG. Polymorphic ventricular tachycardia with a normal QT interval following azithromycin. Pacing Clin Electrophysiol. 2005;28(11):1221-1222.

3. Rao GA, Mann JR, Shoaibi A, et al. Azithromycin and levofloxacin use and increased risk of cardiac arrhythmia and death. Annals of family medicine. 2014;12(2):121-127.

4. Ray WA, Murray KT, Hall K, Arbogast PG, Stein CM. Azithromycin and the risk of cardiovascular death. N Engl J Med. 2012;366(20):1881-1890.

5. Chou HW, Wang JL, Chang CH, Lai CL, Lai MS, Chan KA. Risks of cardiac arrhythmia and mortality among patients using new-generation macrolides, fluoroquinolones, and beta-lactam/beta-lactamase inhibitors: a Taiwanese nationwide study. Clinical infectious diseases : an official publication of the Infectious Diseases Society of America. 2015;60(4):566-577.

6. U.S. Department of Health and Human Services USFDA. FDA Drug Safety Communication: Azithromycin (Zithromax or Zmax) and the risk of potentially fatal heart rhythms. 2013; http://www.fda.gov/Drugs/DrugSafety/ucm341822.htm. Accessed September 20, 2016.

7. Trac MH, McArthur E, Jandoc R, et al. Macrolide antibiotics and the risk of ventricular arrhythmia in older adults. CMAJ. 2016;188(7):E120-129.

8. Svanstrom H, Pasternak B, Hviid A. Use of azithromycin and death from cardiovascular causes. N Engl J Med. 2013;368(18):1704-1712.

9. Mortensen EM, Halm EA, Pugh MJ, et al. Association of azithromycin with mortality and cardiovascular events among older patients hospitalized with pneumonia. JAMA. 2014;311(21):2199-2208.

10. Ray WA, Murray KT, Meredith S, Narasimhulu SS, Hall K, Stein CM. Oral erythromycin and the risk of sudden death from cardiac causes. N Engl J Med. 2004;351(11):1089-1096.

11. Hennessy S, Leonard CE, Freeman CP, et al. Validation of diagnostic codes for outpatient-originating sudden cardiac death and ventricular arrhythmia in Medicaid and Medicare claims data. Pharmacoepidemiology and drug safety. 2010;19(6):555562. 
12. Zambon A, Polo Friz H, Contiero P, Corrao G. Effect of macrolide and fluoroquinolone antibacterials on the risk of ventricular arrhythmia and cardiac arrest: an observational study in Italy using case-control, case-crossover and case-timecontrol designs. Drug Saf. 2009;32(2):159-167.

13. Hennessy S, Leonard CE, Newcomb C, Kimmel SE, Bilker WB. Cisapride and ventricular arrhythmia. British journal of clinical pharmacology. 2008;66(3):375-385.

14. De Bruin ML, van Hemel NM, Leufkens HG, Hoes AW. Hospital discharge diagnoses of ventricular arrhythmias and cardiac arrest were useful for epidemiologic research. Journal of clinical epidemiology. 2005;58(12):1325-1329.

15. Yap YG, Camm AJ. Drug induced QT prolongation and torsades de pointes. Heart. 2003;89(11):1363-1372.

16. Austin PC. The use of propensity score methods with survival or time-to-event outcomes: reporting measures of effect similar to those used in randomized experiments. Stat Med. 2014;33(7):1242-1258.

17. Austin PC. A Tutorial and Case Study in Propensity Score Analysis: An Application to Estimating the Effect of In-Hospital Smoking Cessation Counseling on Mortality. Multivariate Behav Res. 2011;46(1):119-151.

18. Hosmer D.W LS, Sturdivant RX. Applied Logistic Regression. 3rd ed.

19. Rosenbaum PR, Rubin DB. Reducing bias in observational studies using subclassification on the propensity score. Journal of the American Statistical Association. 1984;79(387):516-524.

20. Kleinbaum DG KM. Survival Analysis A Self-Learning Text. 3rd ed.

21. Kestenbaum B, Rudser KD, Shlipak MG, et al. Kidney function, electrocardiographic findings, and cardiovascular events among older adults. Clin J Am Soc Nephrol. 2007;2(3):501-508.

22. Patane S. Torsade de pointes, QT interval prolongation and renal disease. Int J Cardiol. 2011;149(2):241-242.

23. Ma TK, Chow KM, Choy AS, Kwan BC, Szeto CC, Li PK. Clinical manifestation of macrolide antibiotic toxicity in CKD and dialysis patients. Clin Kidney J. 2014;7(6):507-512.

24. Albert RK, Schuller JL, Network CCR. Macrolide antibiotics and the risk of cardiac arrhythmias. Am J Respir Crit Care Med. 2014;189(10):1173-1180.

25. Hancox JC, Hasnain M, Vieweg WV, Crouse EL, Baranchuk A. Azithromycin, cardiovascular risks, QTc interval prolongation, torsade de pointes, and regulatory 
issues: A narrative review based on the study of case reports. Ther Adv Infect Dis. 2013;1(5):155-165.

26. Hancox JC, McPate MJ, El Harchi A, Zhang YH. The hERG potassium channel and hERG screening for drug-induced torsades de pointes. Pharmacol Ther. 2008;119(2):118-132.

27. Finlayson K, Witchel HJ, McCulloch J, Sharkey J. Acquired QT interval prolongation and HERG: implications for drug discovery and development. European journal of pharmacology. 2004;500(1-3):129-142.

28. von Rosensteil NA, Adam D. Macrolide antibacterials. Drug interactions of clinical significance. Drug Saf. 1995;13(2):105-122.

29. Dresser GK, Spence JD, Bailey DG. Pharmacokinetic-pharmacodynamic consequences and clinical relevance of cytochrome P450 3A4 inhibition. Clin Pharmacokinet. 2000;38(1):41-57.

30. Enger C, Cali C, Walker AM. Serious ventricular arrhythmias among users of cisapride and other QT-prolonging agents in the United States. Pharmacoepidemiology and drug safety. 2002;11(6):477-486.

31. Watkins PB. Drug metabolism by cytochromes P450 in the liver and small bowel. Gastroenterol Clin North Am. 1992;21(3):511-526.

32. Liu HH. Safety profile of the fluoroquinolones: focus on levofloxacin. Drug Saf. 2010;33(5):353-369.

33. Briasoulis A, Agarwal V, Pierce WJ. QT prolongation and torsade de pointes induced by fluoroquinolones: infrequent side effects from commonly used medications. Cardiology. 2011;120(2):103-110.

34. Paulussen AD, Aerssens J. Risk factors for drug-induced long-QT syndrome. Neth Heart J. 2005;13(2):47-56.

35. Hoffler D, Koeppe P, Paeske B. Pharmacokinetics of azithromycin in normal and impaired renal function. Infection. 1995;23(6):356-361.

36. Trojak B, Astruc K, Pinoit JM, et al. Hypokalemia is associated with lengthening of QT interval in psychiatric patients on admission. Psychiatry Res. 2009;169(3):257-260.

37. Zanolari Calderari M, Vigier RO, Bettinelli A, Bianchetti MG. Electrocardiographic QT prolongation and sudden death in renal hypokalemic alkalosis. Nephron. 2002;91(4):762-763. 
38. Armahizer MJ, Seybert AL, Smithburger PL, Kane-Gill SL. Drug-drug interactions contributing to QT prolongation in cardiac intensive care units. Journal of critical care. 2013;28(3):243-249.

39. Austin PC. An Introduction to Propensity Score Methods for Reducing the Effects of Confounding in Observational Studies. Multivariate Behav Res. 2011;46(3):399-424.

40. U.S. Department of Health and Human Services NIoH, National Heart, Lung and Blood Institute. What Are the Signs and Symptoms of Long QT Syndrome? 2011; https://www.nhlbi.nih.gov/health/health-topics/topics/qt/signs\#. Accessed February 24, 2016.

41. Mayo Foundation for Medical Education and Research MC. Long QT syndrome. 2015; http://www.mayoclinic.org/diseases-conditions/long-qtsyndrome/basics/symptoms/con-20025388. Accessed February 24, 2016.

42. Chung CP, Murray KT, Stein CM, Hall K, Ray WA. A computer case definition for sudden cardiac death. Pharmacoepidemiology and drug safety. 2010;19(6):563-572. 
Table 3.1 Demographics and clinical characteristics of the study population

\begin{tabular}{|c|c|c|c|c|}
\hline Characteristics & AMO & AZI & MAC & FLQ \\
\hline Prescriptions (no.) & $2,220,068$ & $1,454,889$ & $1,608,020$ & $1,277,009$ \\
\hline Prescriptions / patient (no.) & 1.7 & 1.7 & 1.8 & 1.8 \\
\hline Mean age \pm SD (year) & $43.7 \pm 14.2$ & $43.5 \pm 14.0$ & $43.6 \pm 13.5$ & $47.2 \pm 14.1$ \\
\hline Female (\%) & 59.2 & 61.8 & 61.7 & 64.6 \\
\hline \multicolumn{5}{|l|}{ Year (\%) } \\
\hline 2011 & 32.6 & 31.5 & 31.9 & 33.5 \\
\hline 2012 & 34.8 & 34.9 & 34.7 & 35.0 \\
\hline 2013 & 32.6 & 33.6 & 33.4 & 31.5 \\
\hline \multicolumn{5}{|l|}{ Month (\%) } \\
\hline January & 10.7 & 10.9 & 10.9 & 10.5 \\
\hline February & 9.7 & 10.0 & 10.0 & 9.5 \\
\hline March & 9.5 & 9.6 & 9.6 & 9.3 \\
\hline April & 8.1 & 8.1 & 8.1 & 8.0 \\
\hline May & 7.9 & 7.8 & 7.8 & 7.9 \\
\hline June & 7.1 & 6.7 & 6.7 & 7.2 \\
\hline July & 6.7 & 6.2 & 6.2 & 6.8 \\
\hline August & 7.1 & 6.7 & 6.7 & 7.3 \\
\hline September & 7.4 & 7.4 & 7.4 & 7.6 \\
\hline October & 8.4 & 8.3 & 8.3 & 8.5 \\
\hline November & 8.8 & 9.1 & 9.1 & 8.8 \\
\hline December & 8.8 & 9.3 & 9.2 & 8.7 \\
\hline \multicolumn{5}{|l|}{ Region (\%) } \\
\hline Northeastern & 10.3 & 13.1 & 12.9 & 10.2 \\
\hline Midwest & 26.7 & 22.8 & 22.6 & 22.6 \\
\hline South & 47.7 & 52.6 & 52.7 & 53.5 \\
\hline West & 15.3 & 11.5 & 11.7 & 13.7 \\
\hline \multicolumn{5}{|l|}{ Comorbidities (\%) } \\
\hline Angina & 0.6 & 0.7 & 0.7 & 0.9 \\
\hline Acute myocardial infarction & 0.6 & 0.5 & 0.5 & 0.8 \\
\hline Other coronary heart disease & 3.4 & 3.3 & 3.3 & 4.8 \\
\hline Heart valve disease & 2.9 & 2.6 & 2.6 & 3.5 \\
\hline Conduction disorder & 0.9 & 0.8 & 0.8 & 1.2 \\
\hline Atrial fibrillation & 1.2 & 1.0 & 1.0 & 1.5 \\
\hline Cardiac arrhythmia & 4.1 & 4.0 & 4.0 & 5.5 \\
\hline Congestive heart failure & 1.1 & 1.0 & 1.0 & 1.7 \\
\hline Hypertension & 23.1 & 23.7 & 23.8 & 30.1 \\
\hline Other cardiovascular disease & 4.2 & 3.9 & 3.9 & 5.4 \\
\hline Hyperlipidemia & 24.9 & 25.7 & 25.8 & 31.7 \\
\hline Hyperkalemia & 0.2 & 0.2 & 0.2 & 0.4 \\
\hline Chronic kidney disease & 1.2 & 1.1 & 1.1 & 2.0 \\
\hline Rheumatic disease & 31.6 & 32.5 & 32.7 & 37.4 \\
\hline Chronic liver disease & 2.4 & 2.5 & 2.5 & 3.7 \\
\hline Chronic lung disease & 8.8 & 11.2 & 11.5 & 13.0 \\
\hline \multicolumn{5}{|l|}{ Medication use history (\%) } \\
\hline $\begin{array}{l}\text { Angiotensin-converting-enzyme } \\
\text { inhibitor }\end{array}$ & 10.4 & 10.1 & 10.2 & 12.9 \\
\hline
\end{tabular}




\begin{tabular}{|c|c|c|c|c|}
\hline Angiotensin receptor blocker & 5.9 & 6.2 & 6.2 & 8.2 \\
\hline Aspirin & 0.4 & 0.4 & 0.4 & 0.5 \\
\hline Sodium-channel blocker & 0.2 & 0.1 & 0.1 & 0.2 \\
\hline Beta-blocker & 9.1 & 8.9 & 8.9 & 11.7 \\
\hline Potassium-channel blocker & 0.3 & 0.2 & 0.2 & 0.4 \\
\hline Calcium-channel blocker & 6.0 & 6.0 & 6.0 & 8.0 \\
\hline Digoxin & 0.3 & 0.2 & 0.2 & 0.3 \\
\hline Loop diuretic & 1.9 & 1.9 & 1.9 & 3.2 \\
\hline Other diuretic & 11.2 & 11.5 & 11.5 & 14.5 \\
\hline Insulin & 1.9 & 1.8 & 1.8 & 2.7 \\
\hline Oral anti-diabetic & 6.2 & 6.2 & 6.2 & 8.2 \\
\hline Beta-agonist & 9.4 & 12.7 & 13.0 & 13.2 \\
\hline Statins & 15.4 & 15.1 & 15.1 & 20.0 \\
\hline Fibrate lipid-lowering agent & 1.9 & 1.9 & 1.9 & 2.6 \\
\hline Nitrate antianginal & 0.8 & 0.8 & 0.8 & 1.2 \\
\hline Other antihypertensive & 2.2 & 2.0 & 2.0 & 4.0 \\
\hline Peripheral vasodilator & 0.2 & 0.2 & 0.2 & 0.3 \\
\hline Platelet inhibitor, none aspirin & 1.1 & 1.1 & 1.1 & 1.6 \\
\hline Tricyclic/tetracyclic antidepressant & 2.3 & 2.3 & 2.3 & 3.2 \\
\hline SSRI/SNRI antidepressant & 11.5 & 12.1 & 12.2 & 14.2 \\
\hline Trazodone antidepressant & 1.8 & 1.8 & 1.8 & 2.4 \\
\hline Other antidepressant & 3.1 & 3.1 & 3.1 & 3.8 \\
\hline Lithium & 0.2 & 0.2 & 0.2 & 0.2 \\
\hline Atypical antipsychotic & 1.2 & 1.1 & 1.1 & 1.5 \\
\hline Other antipsychotic & 0.3 & 0.4 & 0.4 & 0.5 \\
\hline Benzodiazepine anticonvulsant & 6.4 & 7.0 & 7.0 & 8.4 \\
\hline GABA agonist anticonvulsant & 3.1 & 3.1 & 3.1 & 4.6 \\
\hline Hydroxyzine & 0.8 & 1.0 & 1.0 & 1.1 \\
\hline Other bronchodilator & 1.0 & 1.4 & 1.4 & 2.0 \\
\hline \multicolumn{5}{|l|}{ Healthcare utilization (\%) } \\
\hline $\begin{array}{l}\text { Any none study drug prescription } \\
\text { past } 30 \text { days }\end{array}$ & 60.7 & 62.6 & 62.9 & 69.5 \\
\hline Any antibiotic use past 30 days & 7.6 & 7.2 & 7.4 & 9.2 \\
\hline Any outpatient visit past 30 days & 42.0 & 42.7 & 42.9 & 48.0 \\
\hline Any outpatient visits $30-365$ days & 86.6 & 88.7 & 88.9 & 91.2 \\
\hline Any ED visit past 7 days & 1.0 & 1.0 & 1.0 & 1.1 \\
\hline Any hospitalization past 30 days & 0.4 & 0.3 & 0.3 & 0.5 \\
\hline $\begin{array}{l}\text { Any hospitalization past 30-365 } \\
\text { days }\end{array}$ & 7.9 & 7.4 & 7.4 & 10.6 \\
\hline
\end{tabular}

AMO = Amoxicillin, AZI = Azithromycin, ED = Emergency Department, FLQ = Fluoroquinolone Antibiotics, GABA = Gamma-Amino Butyric Acid, oMAC = Other Macrolide Antibiotics; SD = Standard Deviation, SNRI = Serotonin-Norepinephrine Reuptake Inhibitor, SSRI = Selective Serotonin Reuptake Inhibitors 
Figure 3.1 Study cohort selection process

12,774,368 episodes of outpatient oral macrolide antibiotics, fluoroquinolone antibiotics, and amoxicillin identified from January 2011 to December 2013

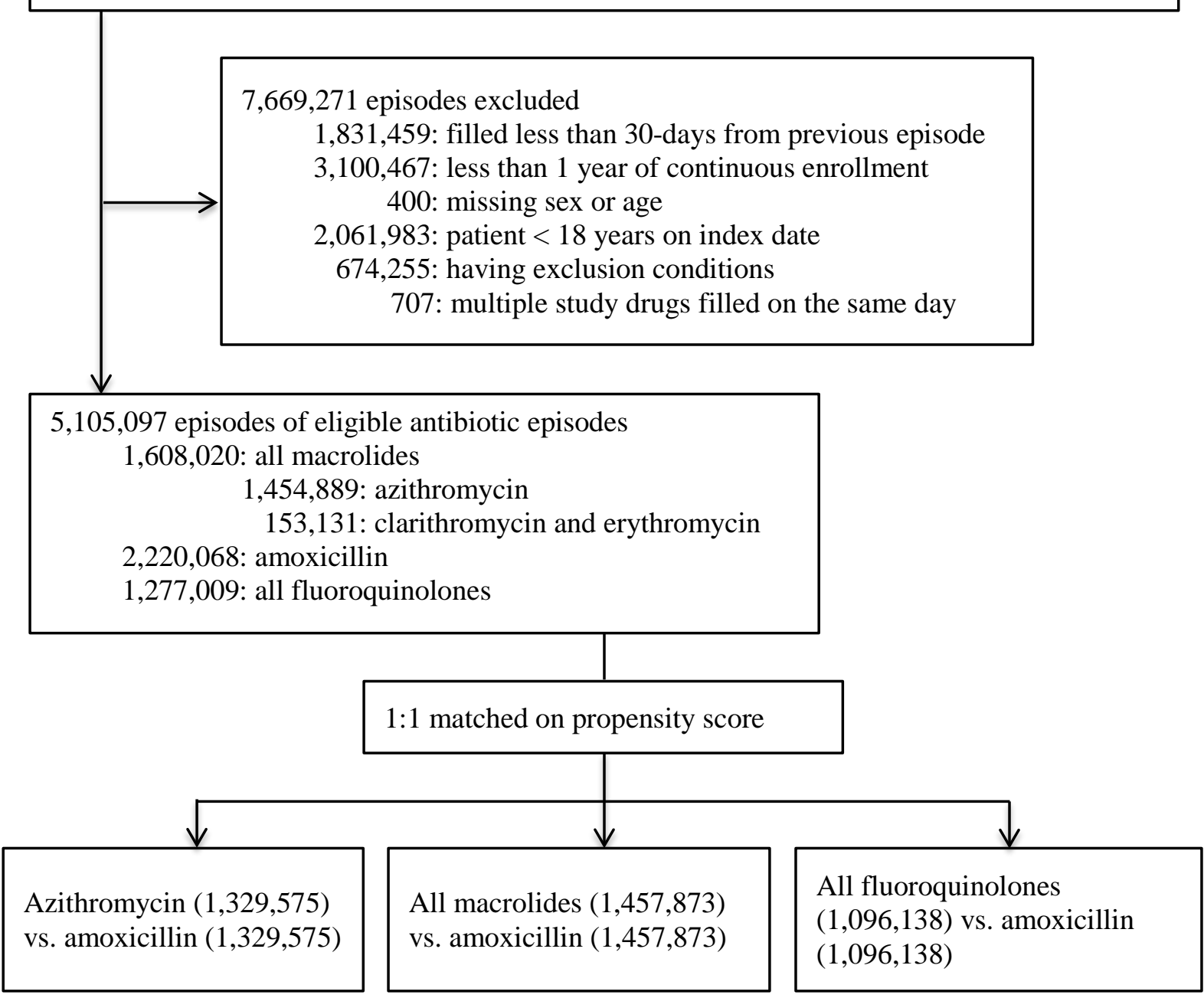


Figure 3.2 Cumulative incidence of ventricular arrhythmia and sudden death with azithromycin and amoxicillin

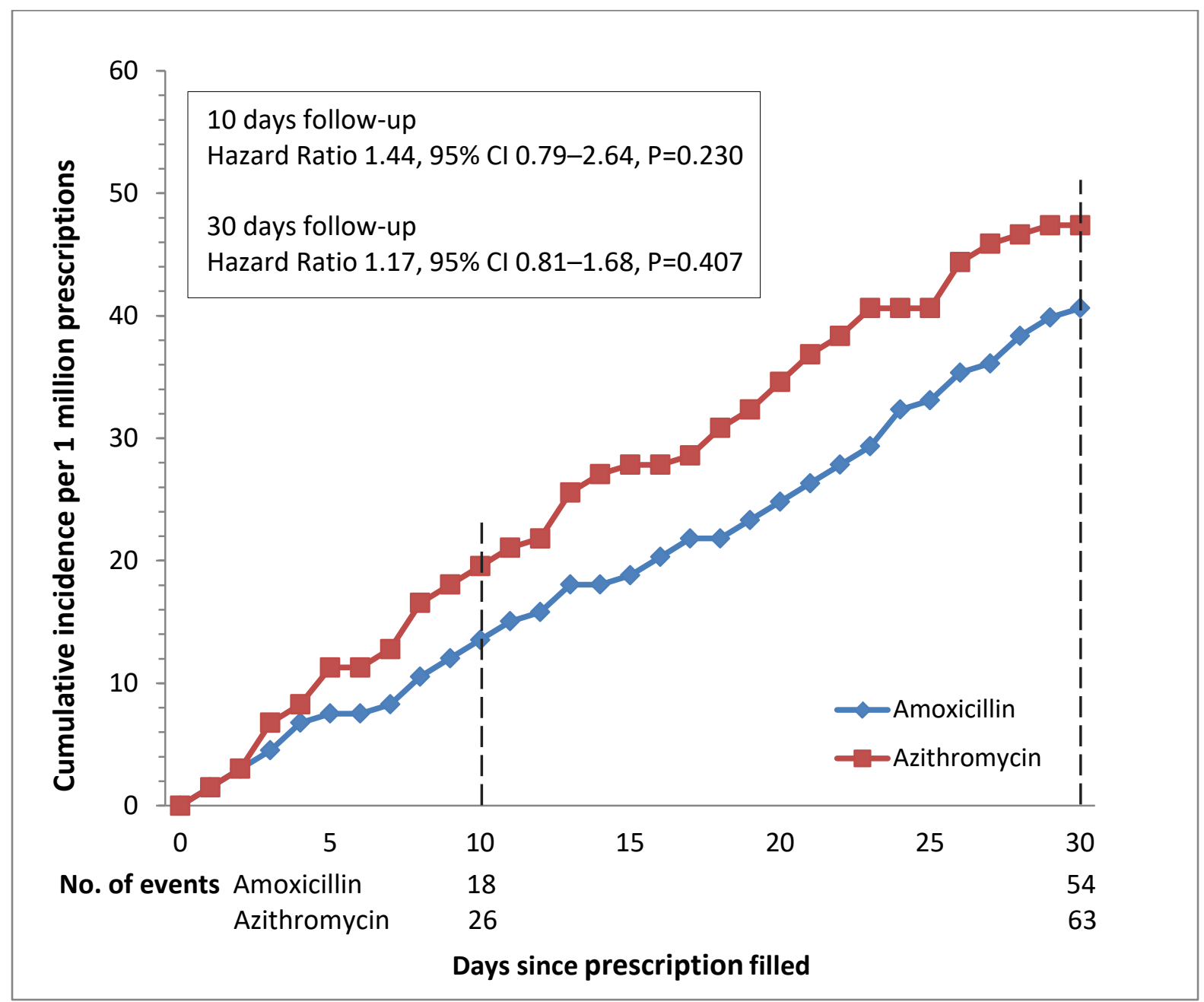


Figure 3.3 Cumulative incidence of ventricular arrhythmia and sudden death with macrolides and amoxicillin

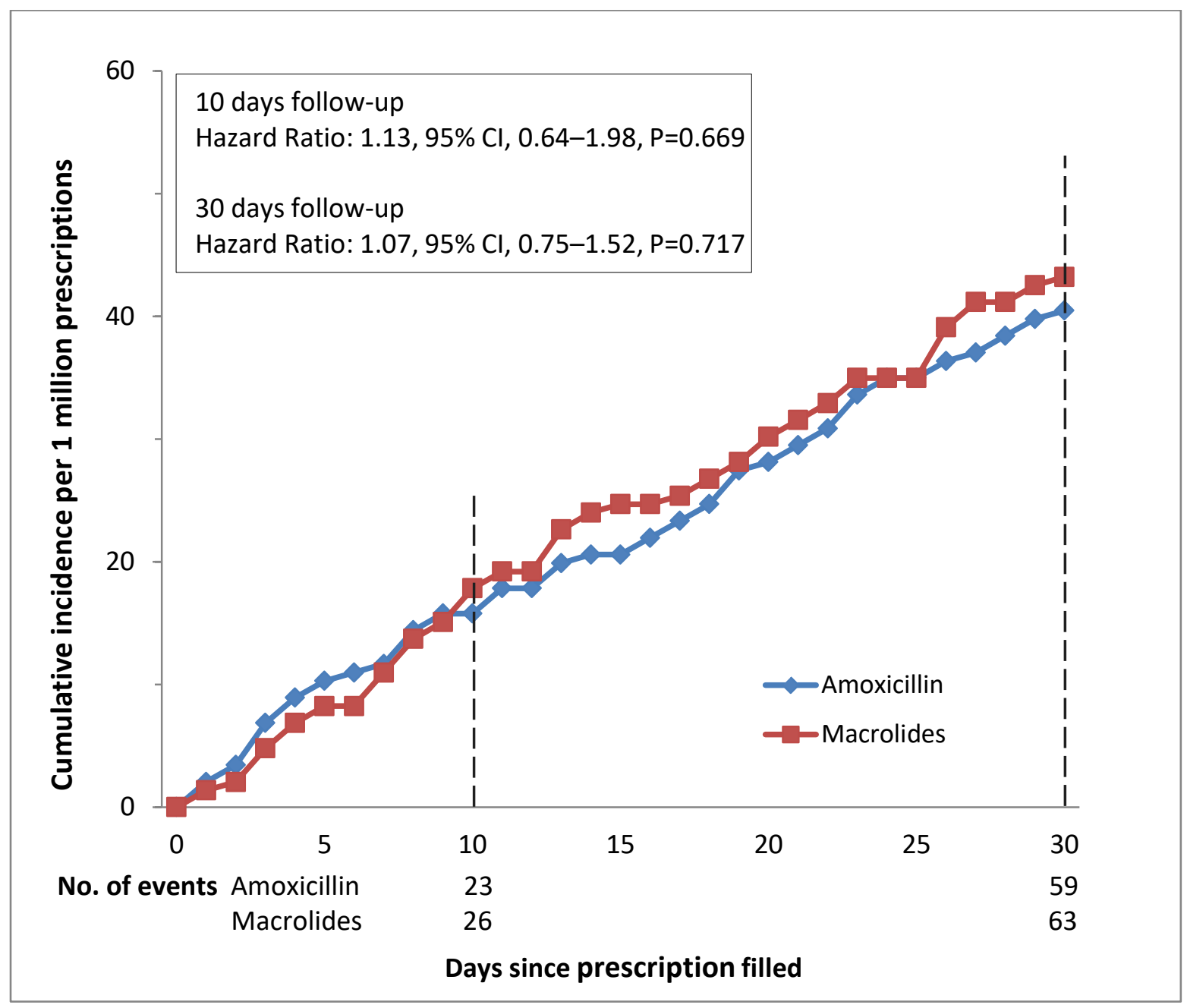


Figure 3.4 Cumulative incidence of ventricular arrhythmia and sudden death with fluoroquinolones and amoxicillin

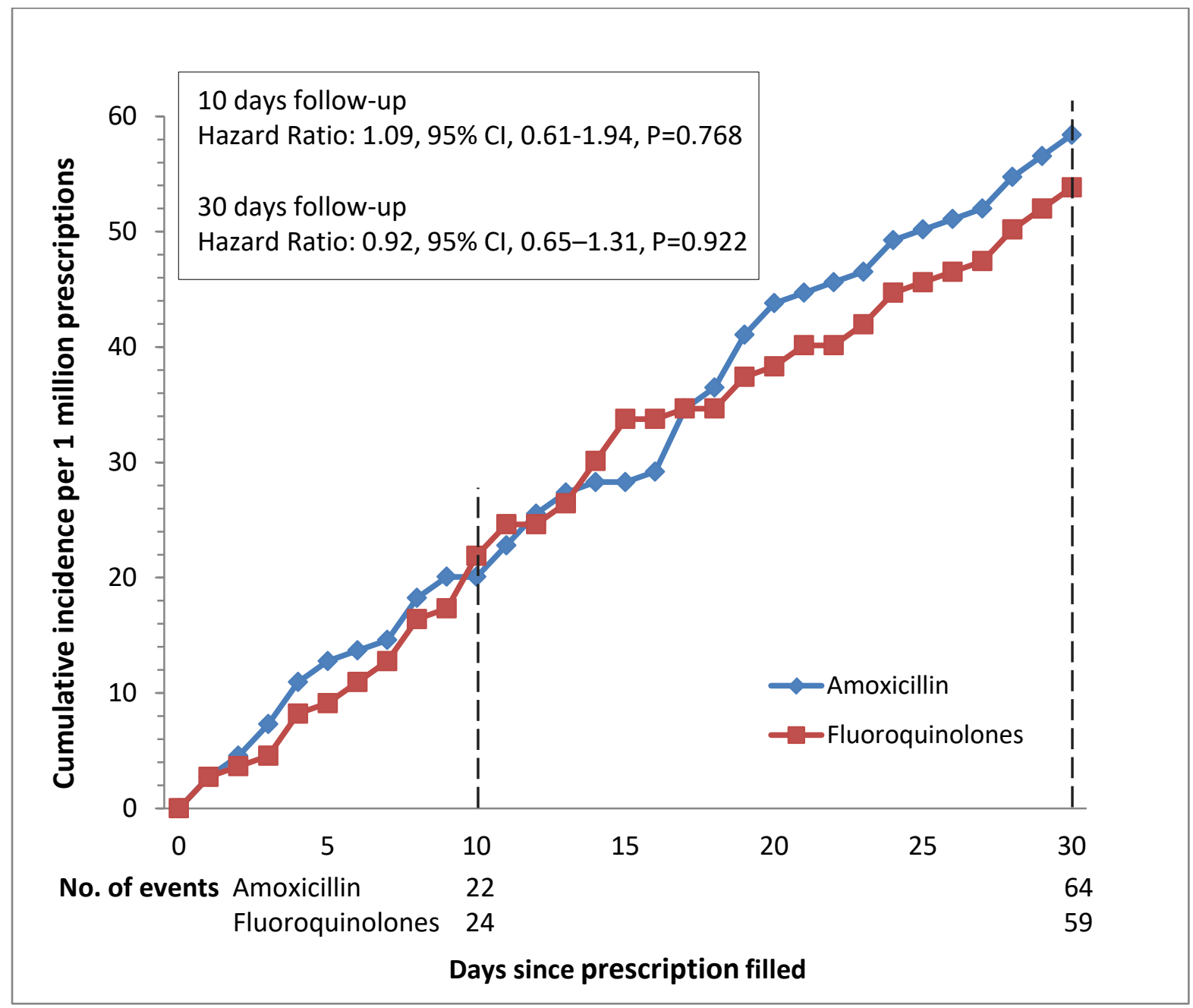


Figure 3.5 Cumulative incidences of ventricular arrhythmia and sudden death with fluoroquinolones and amoxicillin in all diagnoses

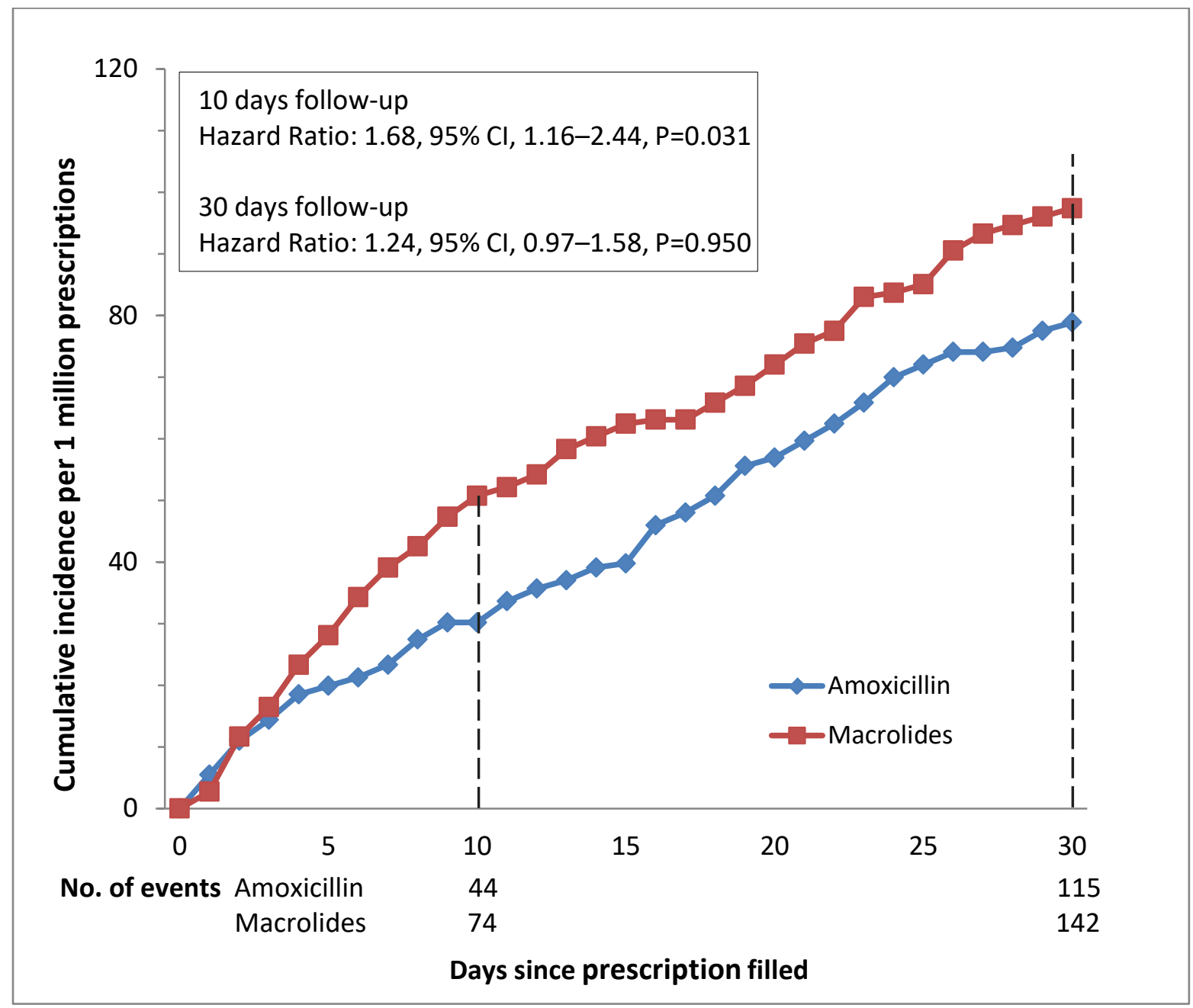




\section{APPENDICES}

Appendix A. List of medications and comorbidities associated with QT prolongation

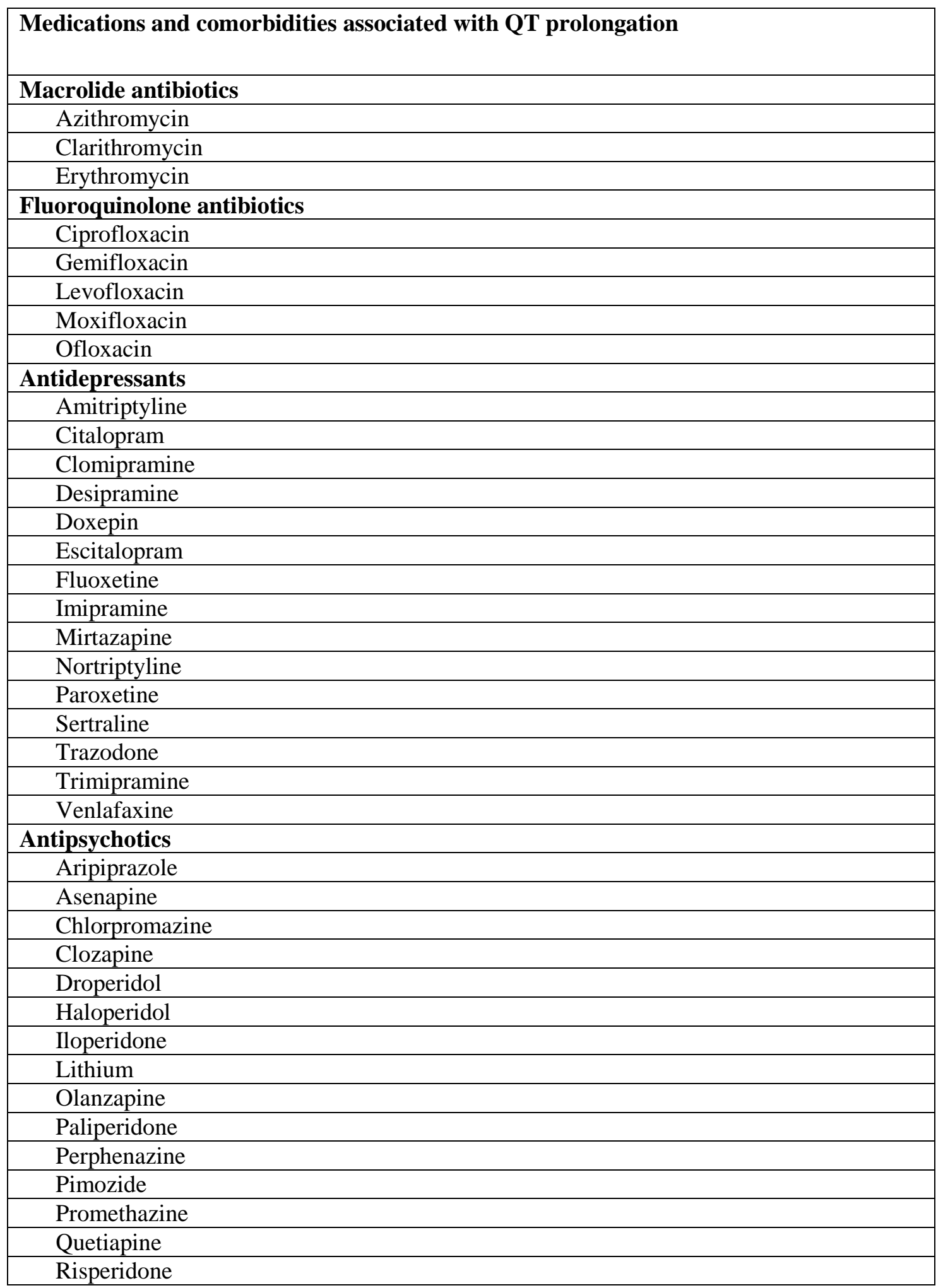




\begin{tabular}{|l|}
\hline Thioridazine \\
\hline Ziprasidone \\
\hline Antihistamines \\
\hline Diphenhydramine \\
\hline Hydroxyzine \\
\hline Antiarrhythmics \\
\hline Amiodarone \\
\hline Disopyramide \\
\hline Dofetilide \\
\hline Dronedarone \\
\hline Flecainide \\
\hline Ibutilide \\
\hline Procainamide \\
\hline Quinidine \\
\hline Sotalol \\
\hline Comorbidities \\
\hline Angina pectoris \\
\hline Cardiac arrhythmia \\
\hline Cardiomyopathy \\
\hline Coronary artery disease (CAD) \\
\hline Heart murmur \\
\hline Heart valve disease (including aortic stenosis), \\
\hline Palpitation \\
\hline Ventricular septal defect (VSD) \\
\hline
\end{tabular}


Appendix B. Exclusion criteria for manuscript III

\begin{tabular}{|c|c|}
\hline Disease & Definition and ICD-9-CM diagnostic codes \\
\hline Cancer & $\begin{array}{l}\text { Diagnosis of cancer (except for non-melanomous skin } \\
\text { cancers) or selected antineoplastic agents. Includes } \\
\text { neoplasms uncertain behavior, ICD9-CM codes 235- } \\
\text { 238, except: } 238.2 \text { (skin), } 238.9 \text { (site unspecified), } \\
\text { 237.70, } 237.71 \text { (neurofibromatosis), } 238.4 \\
\text { (polycythemia v.), } 238.7 \text { (lymphoproliferative disease), } \\
285.22 \text { (anemia in neoplastic disease) }\end{array}$ \\
\hline AIDS & $\begin{array}{l}\text { Diagnosis of AIDS or use of antiretroviral agents or } \\
\text { pentamadine }\end{array}$ \\
\hline Severe Hepatic disease & Diagnoses 570-573 \\
\hline Organ transplant & $\begin{array}{l}\text { Includes kidney, heart, lung, liver, bone marrow, and } \\
\text { pancreas. Includes } 996.8\end{array}$ \\
\hline Serious neuromuscular & $\begin{array}{l}\text { Multiple sclerosis (340), ALS (335.20), Duchenne's } \\
\text { muscular dystrophy (335.21), Huntington's chorea } \\
\text { (333.4), quadriplegia, paraplegia, or spinal cord injury. } \\
\text { Recent stroke (inpatient with primary discharge } \\
\text { diagnosis of 430, 431, 433.x1, 434, 436) with } \\
\text { hemiplegia/hemiparesis }(342,438.2)\end{array}$ \\
\hline $\begin{array}{l}\text { Cardiovascular congenital } \\
\text { anomalies }\end{array}$ & $\begin{array}{l}\text { Common truncus (745.0) transposition great vessels } \\
\text { (745.1), tetrology (745.2), common ventricle (745.3), } \\
\text { endocardial cushion defect (745.6), pulmonary atresia } \\
\text { (746.0), tricuspid atresia (746.1), hypoplastic left heart } \\
\text { (746.7), coarctation of aorta (747.1), other anomalies of } \\
\text { aorta (747.2), total anomalous pulmonary venous } \\
\text { connection (747.41). A single diagnosis is sufficient for } \\
\text { exclusion }\end{array}$ \\
\hline $\begin{array}{l}\text { Other congenital } \\
\text { anomalies/childhood } \\
\text { conditions }\end{array}$ & $\begin{array}{l}\text { Sickle cell (282.6), cerebral palsy (343), spina bifida } \\
\text { (741), Down's syndrome (758.0), hydrocephalus } \\
\text { (742.3), microcephalus (742.1), encephalocele (742.0), } \\
\text { severe mental retardation (318.1, 318.2), cystic fibrosis }\end{array}$ \\
\hline Other end-stage illness & $\begin{array}{l}\text { a. Hospice care. b. Diagnosis of coma, vegetative state, } \\
\text { debility (799.3). c. Total parenteral nutrition, PEG, } \\
\text { enteral feeding, malnutrition (260, 261,262, 263) when } \\
\text { these are for outpatients. d. Gangrene (040, gas } \\
\text { gangrene; } 785.4 \text { gangrene: single diagnosis sufficient) e. } \\
\text { Intravenous medications outside of the hospital, as } \\
\text { indicated by procedures for IV access outside a hospital }\end{array}$ \\
\hline
\end{tabular}




\begin{tabular}{|l|l|}
\hline Drug abuse & stay period \\
& $\begin{array}{l}\text { Includes all medications and drugs with abuse potential } \\
\text { and with the exception of alcohol (unless hospitalization } \\
\text { with primary discharge diagnosis: 291.x, 303.x, 305.0, } \\
980.0,980.9, \text { E860.0, E860.1, E860.9) and tobacco. } \\
\text { Codes are 292.0 (drug withdrawal syndrome), 304.x } \\
\text { (drug dependence), 305.2-305.9 (drug abuse, except } \\
\text { alcohol/tobacco, 305.9 is abuse NOS, may be } \\
\text { nonspecific, but better to exclude), 965.01 (accidental } \\
\text { poisoning, heroin), 969.6 (poisoning, psychodysleptic } \\
\text { [hallucinogens]), 970.81 (cocaine poisoning, added in } \\
\text { 2010), E8500 (heroin poisoning), E8541 } \\
\text { (psychodysleptic poisoning) }\end{array}$ \\
\hline
\end{tabular}


Appendix C. List of covariates for propensity score model

\section{Covariates for propensity score model}

Number of prescriptions for an unique patient

Age

Gender

Year

Month

Geographic region

Angina/angina pectoris

Acute myocardial infarction

Other coronary heart disease

Other cardiovascular disease

Heart valve disease

Conduction disorder

Atrial fibrillation

Cardiac arrhythmia

Congestive heart failure

Hemorrhagic stroke

Ischemic stroke

Transient ischemic attack

Obesity, diagnosed, not morbid

Morbid obesity, diagnosed

Hypertension

Malignant hypertension

Hyperlipidemia

Peripheral vascular disease

Diabetes mellitus

Diabetes, ocular complication

Diabetes, neurologic complication

Diabetes, skin complication

Diabetes, renal complication

Diabetes, other complication

Schizophrenia or other psychotic disorders

Alcohol abuse

Bipolar disorder

Unipolar depression and/or anxiety disorder

Parkinson's Disease

Seizure disorder

Dementia

Other cognitive disorders

Chronic lung disease

COPD and other respiratory conditions

Asthma

Diagnosed smoking

Rheumatic disease

Chronic liver disease

Chronic kidney disease 


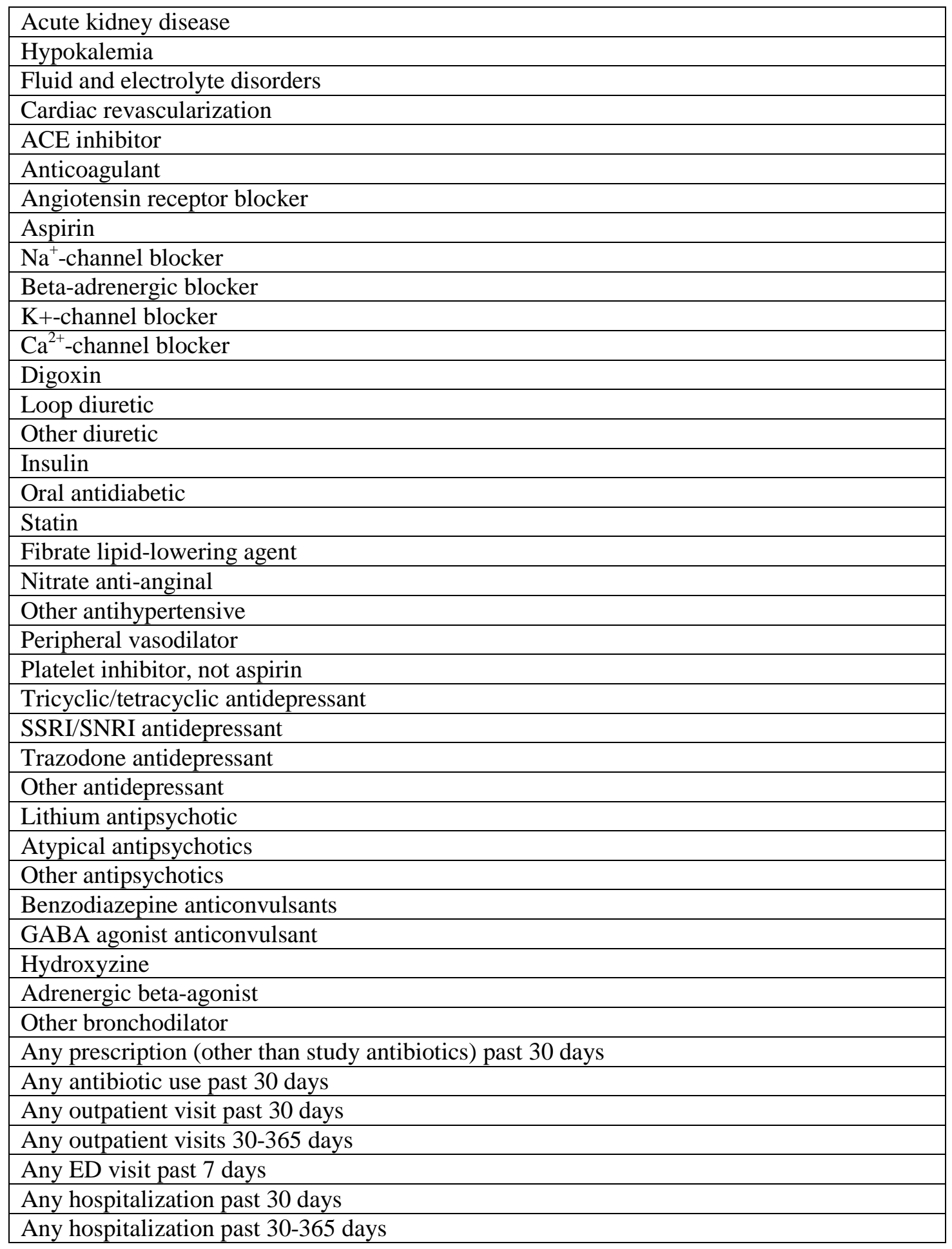

$\mathrm{ACE}=$ angiotensin converting enzyme, $\mathrm{COPD}=$ chronic obstructive pulmonary disease, $\mathrm{ED}=$ emergency department, GABA = gamma-amino butyric acid 
Appendix D. Distributions of propensity scores in matched study cohorts

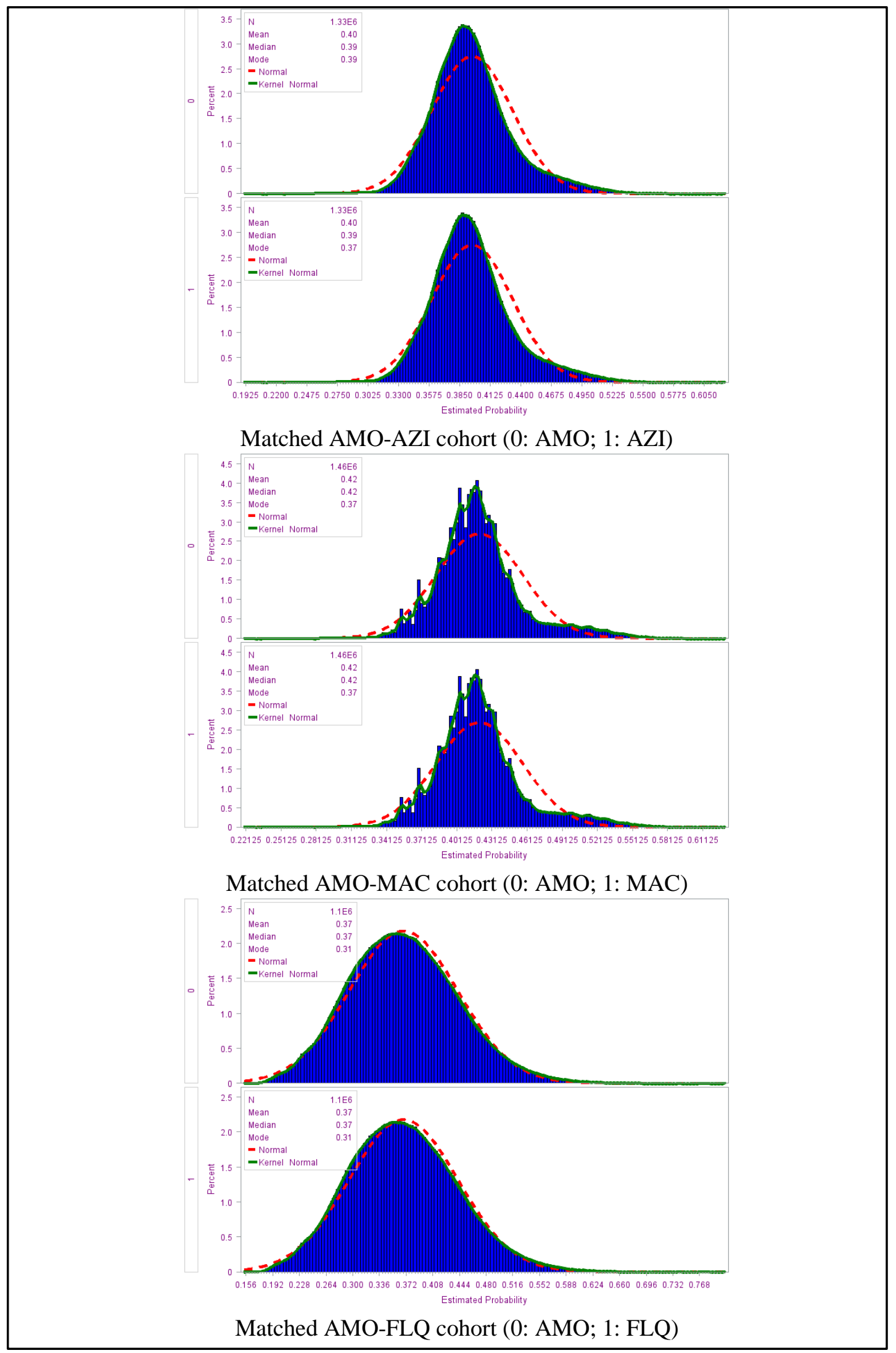


Appendix E. Demographics and clinical characteristics of the matched cohorts

\begin{tabular}{|c|c|c|c|}
\hline \multicolumn{4}{|c|}{$\begin{array}{l}\text { Appendix Table E.1 Demographics and clinical characteristics of the matched AZI- } \\
\text { AMO cohort }\end{array}$} \\
\hline Characteristics & AMO & AZI & d \\
\hline Prescriptions (no.) & $1,329,575$ & $1,329,575$ & 0.000 \\
\hline Mean prescriptions / patient (no.)* & 1.68 & 1.67 & 0.002 \\
\hline Mean age* & 43.0 & 43.1 & 0.009 \\
\hline Female (\%)* & 61.0 & 61.1 & 0.007 \\
\hline \multicolumn{4}{|l|}{ Year $(\%)^{*}$} \\
\hline 2011 & 32.3 & 32.1 & 0.005 \\
\hline 2012 & 34.9 & 34.9 & 0.001 \\
\hline 2013 & 32.8 & 33.0 & 0.004 \\
\hline \multicolumn{4}{|l|}{ Month (\%)* } \\
\hline January & 10.8 & 11.0 & 0.006 \\
\hline February & 9.7 & 10.1 & 0.011 \\
\hline march & 9.5 & 9.6 & 0.004 \\
\hline April & 8.1 & 8.1 & 0.001 \\
\hline May & 8.0 & 7.8 & 0.006 \\
\hline June & 7.0 & 6.7 & 0.011 \\
\hline July & 6.6 & 6.2 & 0.016 \\
\hline August & 7.0 & 6.7 & 0.012 \\
\hline September & 7.4 & 7.3 & 0.001 \\
\hline October & 8.3 & 8.3 & 0.000 \\
\hline November & 8.9 & 9.0 & 0.004 \\
\hline December & 8.8 & 9.2 & 0.015 \\
\hline \multicolumn{4}{|l|}{ Region (\%)* } \\
\hline Northeastern & 11.1 & 12.1 & 0.034 \\
\hline Midwest & 26.3 & 22.7 & 0.084 \\
\hline South & 49.1 & 53.5 & 0.088 \\
\hline West & 13.5 & 11.7 & 0.056 \\
\hline \multicolumn{4}{|l|}{ Comorbidities (\%) } \\
\hline Angina/angina pectoris* & 0.6 & 0.6 & 0.000 \\
\hline Acute myocardial infarction & 0.5 & 0.5 & 0.002 \\
\hline Other coronary heart disease & 3.0 & 3.0 & 0.001 \\
\hline Other cardiovascular disease* & 3.5 & 3.5 & 0.002 \\
\hline Heart valve disease* & 2.3 & 2.4 & 0.002 \\
\hline Conduction disorder* & 0.7 & 0.7 & 0.000 \\
\hline Atrial fibrillation & 0.8 & 0.7 & 0.004 \\
\hline Cardiac arrhythmia* & 3.5 & 3.5 & 0.000 \\
\hline Congestive heart failure & 0.9 & 0.9 & 0.001 \\
\hline Hemorrhagic stroke & 0.0 & 0.0 & 0.001 \\
\hline Ischemic stroke & 0.3 & 0.3 & 0.002 \\
\hline Transient ischemic attack & 0.3 & 0.3 & 0.002 \\
\hline Obesity, diagnosed, not morbid & 5.0 & 5.0 & 0.000 \\
\hline Morbid obesity, diagnosed* & 1.6 & 1.6 & 0.002 \\
\hline Hypertension* & 22.0 & 22.5 & 0.011 \\
\hline Malignant hypertension* & 0.8 & 0.8 & 0.002 \\
\hline Hyperlipidemia* & 24.4 & 24.5 & 0.003 \\
\hline
\end{tabular}




\begin{tabular}{|c|c|c|c|}
\hline Peripheral vascular disease* & 1.3 & 1.3 & 0.001 \\
\hline Diabetes mellitus & 10.9 & 11.0 & 0.003 \\
\hline Diabetes, ocular complication & 0.5 & 0.5 & 0.001 \\
\hline Diabetes, neurologic complication* & 0.6 & 0.6 & 0.001 \\
\hline Diabetes, skin complication* & 0.2 & 0.2 & 0.000 \\
\hline Diabetes, renal complication & 0.3 & 0.3 & 0.000 \\
\hline Diabetes, other complication & 0.5 & 0.5 & 0.001 \\
\hline Schizophrenia or other psychotic disorders & 0.2 & 0.2 & 0.002 \\
\hline Alcohol abuse* & 0.4 & 0.4 & 0.000 \\
\hline Bipolar disorder* & 0.9 & 0.9 & 0.001 \\
\hline Unipolar depression and/or anxiety disorder & 14.6 & 14.7 & 0.002 \\
\hline Parkinson's Disease* & 0.1 & 0.1 & 0.001 \\
\hline Seizure disorder & 0.7 & 0.7 & 0.003 \\
\hline Dementia & 0.1 & 0.1 & 0.000 \\
\hline Other cognitive disorders & 0.2 & 0.2 & 0.001 \\
\hline Chronic lung disease* & 8.5 & 8.6 & 0.003 \\
\hline COPD and other respiratory conditions* & 1.7 & 1.7 & 0.001 \\
\hline Asthma* & 6.3 & 6.4 & 0.003 \\
\hline Diagnosed smoking* & 3.8 & 3.9 & 0.006 \\
\hline Rheumatic disease & 31.3 & 31.5 & 0.004 \\
\hline Chronic liver disease & 2.4 & 2.4 & 0.002 \\
\hline Chronic kidney disease* & 1.2 & 1.3 & 0.001 \\
\hline Hypokalemia & 0.2 & 0.2 & 0.000 \\
\hline Fluid and electrolyte disorders & 0.2 & 0.2 & 0.000 \\
\hline Cardiac revascularization & 0.0 & 0.0 & 0.000 \\
\hline \multicolumn{4}{|l|}{ Medication use history (\%) } \\
\hline ACE inhibitor* & 9.6 & 9.7 & 0.006 \\
\hline Anticoagulant* & 0.8 & 0.8 & 0.001 \\
\hline Angiotensin receptor blocker* & 5.7 & 5.8 & 0.003 \\
\hline Aspirin & 0.4 & 0.4 & 0.001 \\
\hline $\mathrm{Na}^{+}$-channel blocker* & 0.1 & 0.1 & 0.002 \\
\hline Beta-adrenergic blocker* & 8.3 & 8.4 & 0.004 \\
\hline K+-channel blocker* & 0.1 & 0.1 & 0.004 \\
\hline $\mathrm{Ca}^{2+}$-channel blocker & 5.6 & 5.6 & 0.001 \\
\hline Digoxin* & 0.1 & 0.1 & 0.002 \\
\hline Loop diuretic & 1.7 & 1.7 & 0.002 \\
\hline Other diuretic* & 10.8 & 10.9 & 0.004 \\
\hline Insulin* & 1.7 & 1.7 & 0.000 \\
\hline Oral antidiabetic* & 5.8 & 5.9 & 0.004 \\
\hline Statin* & 14.4 & 14.5 & 0.003 \\
\hline Fibrate lipid-lowering agent & 1.8 & 1.8 & 0.000 \\
\hline Nitrate anti-anginal* & 0.7 & 0.7 & 0.001 \\
\hline Other antihypertensive* & 1.9 & 1.9 & 0.000 \\
\hline Peripheral vasodilator & 0.2 & 0.2 & 0.001 \\
\hline Platelet inhibitor, not aspirin & 1.0 & 1.0 & 0.002 \\
\hline Tricyclic/tetracyclic antidepressant & 2.2 & 2.2 & 0.006 \\
\hline SSRI/SNRI antidepressant & 11.6 & 11.7 & 0.004 \\
\hline Trazodone antidepressant & 1.8 & 1.7 & 0.005 \\
\hline Other antidepressant* & 3.0 & 3.0 & 0.000 \\
\hline
\end{tabular}




\begin{tabular}{|l|c|c|c|}
\hline Lithium antipsychotic* & 0.2 & 0.2 & 0.001 \\
\hline Atypical antipsychotics* & 1.1 & 1.1 & 0.002 \\
\hline Other antipsychotics & 0.3 & 0.3 & 0.001 \\
\hline Benzodiazepine anticonvulsants* & 6.4 & 6.4 & 0.001 \\
\hline GABA agonist anticonvulsant & 2.9 & 2.9 & 0.002 \\
\hline Hydroxyzine* & 0.9 & 0.9 & 0.000 \\
\hline Adrenergic beta-agonist* & 9.4 & 9.4 & 0.003 \\
\hline Other bronchodilator* & 0.9 & 0.9 & 0.000 \\
\hline Healthcare Utilization (\%) & & & \\
\hline $\begin{array}{l}\text { Any prescription (other than study ABX) past 30 } \\
\text { days* }\end{array}$ & 60.2 & 60.8 & 0.013 \\
\hline Any antibiotic use past 30 days* & 6.9 & 7.1 & 0.007 \\
\hline Any outpatient visit past 30 days & 41.5 & 41.7 & 0.003 \\
\hline Any outpatient visits 30-365 days* & 88.4 & 88.0 & 0.010 \\
\hline Any ED visit past 7 days & 1.0 & 1.0 & 0.000 \\
\hline Any hospitalization past 30 days* & 0.3 & 0.3 & 0.000 \\
\hline Any hospitalization past 30-365 days* & 7.1 & 7.0 & 0.003 \\
\hline
\end{tabular}

* Covariates included the propensity score model

C-statistic: 0.547

Hosmer and Lemeshow Goodness-of-Fit (GOF) test: $\chi^{2}=121.7$ (degree of freedom $[\mathrm{df}]=8$, pvalue $<0.0001$ )

$\mathrm{ABX}=$ antibiotics, $\mathrm{ACE}=$ angiotensin converting enzyme, $\mathrm{AMO}=$ amoxicillin, $\mathrm{AZI}=$ azithromycin, $\mathrm{COPD}$ = chronic obstructive pulmonary disease, $\mathrm{ED}=$ emergency department, GABA = gamma-amino butyric acid 


\begin{tabular}{|c|c|c|c|}
\hline \multicolumn{4}{|c|}{$\begin{array}{l}\text { Appendix Table E.2 Demographics and clinical characteristics of the matched MAC- } \\
\text { AMO cohort }\end{array}$} \\
\hline Characteristics & AMO & MAC & d \\
\hline Prescriptions (no.) & $1,457,873$ & $1,457,873$ & 0.000 \\
\hline Mean prescriptions / patient (no.)* & 1.7 & 1.7 & 0.037 \\
\hline Mean age* & 43.0 & 43.0 & 0.004 \\
\hline Female (\%)* & 61.6 & 61.4 & 0.005 \\
\hline \multicolumn{4}{|l|}{ Year $(\%)$} \\
\hline 2011 & 31.5 & 32.0 & 0.013 \\
\hline 2012 & 34.9 & 34.8 & 0.002 \\
\hline 2013 & 33.6 & 33.2 & 0.010 \\
\hline \multicolumn{4}{|l|}{ Month (\%)* } \\
\hline January & 10.8 & 10.9 & 0.005 \\
\hline February & 9.9 & 10.0 & 0.005 \\
\hline march & 9.6 & 9.6 & 0.001 \\
\hline April & 8.1 & 8.1 & 0.000 \\
\hline May & 7.9 & 7.8 & 0.001 \\
\hline June & 6.9 & 6.8 & 0.006 \\
\hline July & 6.4 & 6.3 & 0.008 \\
\hline August & 6.9 & 6.7 & 0.006 \\
\hline September & 7.4 & 7.4 & 0.002 \\
\hline October & 8.3 & 8.3 & 0.000 \\
\hline November & 9.0 & 9.0 & 0.003 \\
\hline December & 9.0 & 9.2 & 0.005 \\
\hline \multicolumn{4}{|l|}{ Region (\%)* } \\
\hline Northeastern & 11.6 & 12.2 & 0.021 \\
\hline Midwest & 24.2 & 22.7 & 0.044 \\
\hline South & 51.3 & 53.3 & 0.058 \\
\hline West & 12.9 & 11.9 & 0.038 \\
\hline \multicolumn{4}{|l|}{ Comorbidities (\%) } \\
\hline Angina/angina pectoris* & 0.5 & 0.5 & 0.001 \\
\hline Acute myocardial infarction* & 0.4 & 0.4 & 0.002 \\
\hline Other coronary heart disease* & 2.7 & 2.7 & 0.001 \\
\hline Other cardiovascular disease* & 3.1 & 3.2 & 0.001 \\
\hline Heart valve disease* & 2.1 & 2.1 & 0.002 \\
\hline Conduction disorder* & 0.6 & 0.6 & 0.001 \\
\hline Atrial fibrillation* & 0.7 & 0.6 & 0.002 \\
\hline Cardiac arrhythmia* & 3.2 & 3.2 & 0.002 \\
\hline Congestive heart failure & 0.8 & 0.8 & 0.002 \\
\hline Hemorrhagic stroke & 0.0 & 0.0 & 0.000 \\
\hline Ischemic stroke* & 0.3 & 0.3 & 0.000 \\
\hline Transient ischemic attack & 0.3 & 0.3 & 0.003 \\
\hline Obesity, diagnosed, not morbid & 4.8 & 4.8 & 0.001 \\
\hline Morbid obesity, diagnosed* & 1.6 & 1.5 & 0.001 \\
\hline Hypertension* & 21.1 & 21.2 & 0.003 \\
\hline Malignant hypertension* & 0.7 & 0.7 & 0.002 \\
\hline Hyperlipidemia* & 23.6 & 23.5 & 0.001 \\
\hline Peripheral vascular disease* & 1.2 & 1.2 & 0.000 \\
\hline Diabetes mellitus* & 10.3 & 10.3 & 0.001 \\
\hline
\end{tabular}




\begin{tabular}{|c|c|c|c|}
\hline Diabetes, ocular complication* & 0.4 & 0.4 & 0.000 \\
\hline Diabetes, neurologic complication & 0.5 & 0.5 & 0.003 \\
\hline Diabetes, skin complication* & 0.2 & 0.2 & 0.002 \\
\hline Diabetes, renal complication* & 0.2 & 0.2 & 0.001 \\
\hline Diabetes, other complication & 0.5 & 0.5 & 0.001 \\
\hline Schizophrenia or other psychotic disorders & 0.2 & 0.2 & 0.001 \\
\hline Alcohol abuse* & 0.4 & 0.4 & 0.002 \\
\hline Bipolar disorder & 0.8 & 0.8 & 0.002 \\
\hline Unipolar depression and/or anxiety disorder & 14.3 & 14.5 & 0.004 \\
\hline Parkinson's Disease & 0.1 & 0.1 & 0.000 \\
\hline Seizure disorder & 0.7 & 0.7 & 0.004 \\
\hline Dementia* & 0.0 & 0.0 & 0.000 \\
\hline Other cognitive disorders & 0.1 & 0.1 & 0.001 \\
\hline Chronic lung disease* & 8.5 & 8.5 & 0.002 \\
\hline COPD and other respiratory conditions* & 1.5 & 1.5 & 0.002 \\
\hline Asthma* & 6.5 & 6.6 & 0.002 \\
\hline Diagnosed smoking* & 3.5 & 3.6 & 0.007 \\
\hline Rheumatic disease* & 30.7 & 31.1 & 0.007 \\
\hline Chronic liver disease* & 2.2 & 2.2 & 0.003 \\
\hline Chronic kidney disease* & 1.0 & 1.0 & 0.002 \\
\hline Hypokalemia & 0.2 & 0.2 & 0.001 \\
\hline Fluid and electrolyte disorders & 0.2 & 0.2 & 0.001 \\
\hline Cardiac revascularization* & 0.0 & 0.0 & 0.001 \\
\hline \multicolumn{4}{|l|}{ Medication use history (\%) } \\
\hline ACE inhibitor* & 9.1 & 9.2 & 0.003 \\
\hline Anticoagulant* & 0.7 & 0.7 & 0.001 \\
\hline Angiotensin receptor blocker* & 5.3 & 5.4 & 0.003 \\
\hline Aspirin & 0.4 & 0.4 & 0.001 \\
\hline $\mathrm{Na}^{+}$-channel blocker* & 0.1 & 0.1 & 0.003 \\
\hline Beta-adrenergic blocker* & 7.7 & 7.8 & 0.002 \\
\hline K+-channel blocker* & 0.1 & 0.1 & 0.002 \\
\hline $\mathrm{Ca}^{2+}$-channel blocker* & 5.3 & 5.3 & 0.003 \\
\hline Digoxin & 0.1 & 0.1 & 0.001 \\
\hline Loop diuretic* & 1.6 & 1.6 & 0.002 \\
\hline Other diuretic* & 10.2 & 10.3 & 0.004 \\
\hline Insulin* & 1.5 & 1.6 & 0.002 \\
\hline Oral antidiabetic & 5.6 & 5.6 & 0.001 \\
\hline Statin* & 13.8 & 13.7 & 0.002 \\
\hline Fibrate lipid-lowering agent* & 1.7 & 1.7 & 0.000 \\
\hline Nitrate anti-anginal & 0.6 & 0.6 & 0.003 \\
\hline Other antihypertensive* & 1.8 & 1.8 & 0.002 \\
\hline Peripheral vasodilator* & 0.1 & 0.1 & 0.000 \\
\hline Platelet inhibitor, not aspirin* & 0.9 & 0.9 & 0.001 \\
\hline Tricyclic/tetracyclic antidepressant* & 2.0 & 2.0 & 0.005 \\
\hline SSRI/SNRI antidepressant* & 11.4 & 11.5 & 0.003 \\
\hline Trazodone antidepressant* & 1.7 & 1.7 & 0.005 \\
\hline Other antidepressant* & 2.8 & 2.8 & 0.002 \\
\hline Lithium antipsychotic* & 0.1 & 0.2 & 0.002 \\
\hline Atypical antipsychotics* & 1.0 & 1.0 & 0.001 \\
\hline
\end{tabular}




\begin{tabular}{|l|c|c|c|}
\hline Other antipsychotics* & 0.3 & 0.3 & 0.001 \\
\hline Benzodiazepine anticonvulsants* & 6.0 & 6.1 & 0.004 \\
\hline GABA agonist anticonvulsant* & 2.6 & 2.7 & 0.003 \\
\hline Hydroxyzine* & 0.8 & 0.8 & 0.002 \\
\hline Adrenergic beta-agonist* & 9.6 & 9.6 & 0.000 \\
\hline Other bronchodilator* & 0.8 & 0.8 & 0.001 \\
\hline Healthcare Utilization (\%) & & & \\
\hline $\begin{array}{l}\text { Any prescription (other than study ABX) past 30 } \\
\text { days* }\end{array}$ & 60.8 & 60.6 & 0.004 \\
\hline Any antibiotic use past 30 days* & 7.3 & 7.1 & 0.006 \\
\hline Any outpatient visit past 30 days* & 41.5 & 41.4 & 0.001 \\
\hline Any outpatient visits 30-365 days* & 88.1 & 88.0 & 0.001 \\
\hline Any ED visit past 7 days & 1.0 & 1.0 & 0.003 \\
\hline Any hospitalization past 30 days & 0.3 & 0.3 & 0.001 \\
\hline Any hospitalization past 30-365 days* & 6.6 & 6.6 & 0.003 \\
\hline
\end{tabular}

* Covariates included the propensity score model

C-statistic: 0.546

Hosmer and Lemeshow Goodness-of-Fit (GOF) test: $\chi^{2}=153.7$ (df $=8$, p-value $\left.<0.0001\right)$ $\mathrm{ABX}=$ antibiotics, $\mathrm{ACE}=$ angiotensin converting enzyme, $\mathrm{AMO}=$ amoxicillin, $\mathrm{COPD}=$ chronic obstructive pulmonary disease, $\mathrm{ED}=$ emergency department, GABA = gamma-amino butyric acid, MAC = macrolide antibiotics 


\begin{tabular}{|c|c|c|c|}
\hline \multicolumn{4}{|c|}{$\begin{array}{l}\text { Appendix Table E.3 Demographics and clinical characteristics of the matched FLQ- } \\
\text { AMO cohort }\end{array}$} \\
\hline Characteristics & AMO & FLQ & d \\
\hline Prescriptions (no.) & $1,096,138$ & $1,096,138$ & 0.000 \\
\hline Mean prescriptions / patient (no.)* & 1.7 & 1.7 & 0.005 \\
\hline Mean age* & 45.6 & 45.7 & 0.007 \\
\hline Female (\%)* & 63.8 & 64.1 & 0.006 \\
\hline \multicolumn{4}{|l|}{ Year $(\%)$} \\
\hline 2011 & 33.9 & 33.5 & 0.008 \\
\hline 2012 & 35.0 & 34.9 & 0.001 \\
\hline 2013 & 31.1 & 31.5 & 0.009 \\
\hline \multicolumn{4}{|l|}{ Month (\%)* } \\
\hline January & 10.8 & 10.7 & 0.003 \\
\hline February & 9.8 & 9.6 & 0.007 \\
\hline march & 9.5 & 9.4 & 0.003 \\
\hline April & 8.1 & 8.1 & 0.003 \\
\hline May & 7.8 & 7.9 & 0.003 \\
\hline June & 7.0 & 7.1 & 0.005 \\
\hline July & 6.7 & 6.8 & 0.002 \\
\hline August & 7.1 & 7.2 & 0.003 \\
\hline September & 7.4 & 7.6 & 0.004 \\
\hline October & 8.4 & 8.4 & 0.000 \\
\hline November & 8.7 & 8.8 & 0.001 \\
\hline December & 8.7 & 8.7 & 0.000 \\
\hline \multicolumn{4}{|l|}{ Region (\%)* } \\
\hline Northeastern & 9.7 & 10.4 & 0.025 \\
\hline Midwest & 25.5 & 22.7 & 0.065 \\
\hline South & 49.3 & 53.4 & 0.081 \\
\hline West & 15.5 & 13.5 & 0.057 \\
\hline \multicolumn{4}{|l|}{ Comorbidities (\%) } \\
\hline Angina/angina pectoris* & 0.7 & 0.7 & 0.001 \\
\hline Acute myocardial infarction* & 0.6 & 0.6 & 0.000 \\
\hline Other coronary heart disease* & 3.8 & 3.8 & 0.000 \\
\hline Other cardiovascular disease* & 4.5 & 4.5 & 0.002 \\
\hline Heart valve disease* & 3.2 & 3.1 & 0.004 \\
\hline Conduction disorder* & 1.0 & 0.9 & 0.001 \\
\hline Atrial fibrillation* & 1.3 & 1.3 & 0.003 \\
\hline Cardiac arrhythmia* & 4.5 & 4.5 & 0.003 \\
\hline Congestive heart failure & 1.2 & 1.2 & 0.001 \\
\hline Hemorrhagic stroke & 0.0 & 0.0 & 0.001 \\
\hline Ischemic stroke* & 0.4 & 0.4 & 0.001 \\
\hline Transient ischemic attack & 0.4 & 0.4 & 0.000 \\
\hline Obesity, diagnosed, not morbid & 5.5 & 5.5 & 0.001 \\
\hline Morbid obesity, diagnosed* & 1.9 & 1.9 & 0.001 \\
\hline Hypertension* & 26.4 & 26.2 & 0.004 \\
\hline Malignant hypertension* & 0.9 & 1.0 & 0.001 \\
\hline Hyperlipidemia* & 28.6 & 28.3 & 0.006 \\
\hline Peripheral vascular disease* & 1.7 & 1.7 & 0.001 \\
\hline Diabetes mellitus* & 13.0 & 13.0 & 0.001 \\
\hline
\end{tabular}




\begin{tabular}{|c|c|c|c|}
\hline Diabetes, ocular complication* & 0.6 & 0.6 & 0.000 \\
\hline Diabetes, neurologic complication & 0.8 & 0.8 & 0.002 \\
\hline Diabetes, skin complication* & 0.3 & 0.3 & 0.002 \\
\hline Diabetes, renal complication* & 0.4 & 0.4 & 0.001 \\
\hline Diabetes, other complication & 0.7 & 0.7 & 0.002 \\
\hline Schizophrenia or other psychotic disorders & 0.2 & 0.2 & 0.001 \\
\hline Alcohol abuse* & 0.5 & 0.5 & 0.000 \\
\hline Bipolar disorder & 1.1 & 1.0 & 0.002 \\
\hline Unipolar depression and/or anxiety disorder & 16.0 & 15.9 & 0.003 \\
\hline Parkinson's Disease & 0.1 & 0.1 & 0.002 \\
\hline Seizure disorder & 0.8 & 0.8 & 0.002 \\
\hline Dementia* & 0.1 & 0.1 & 0.000 \\
\hline Other cognitive disorders & 0.2 & 0.2 & 0.001 \\
\hline Chronic lung disease* & 9.5 & 9.7 & 0.007 \\
\hline COPD and other respiratory conditions* & 1.9 & 2.0 & 0.004 \\
\hline Asthma* & 7.0 & 7.2 & 0.006 \\
\hline Diagnosed smoking* & 4.3 & 4.3 & 0.001 \\
\hline Rheumatic disease* & 35.6 & 34.5 & 0.023 \\
\hline Chronic liver disease* & 2.7 & 2.8 & 0.004 \\
\hline Chronic kidney disease* & 1.4 & 1.5 & 0.002 \\
\hline Hypokalemia & 0.3 & 0.3 & 0.001 \\
\hline Fluid and electrolyte disorders & 0.3 & 0.3 & 0.001 \\
\hline Cardiac revascularization* & 0.1 & 0.1 & 0.000 \\
\hline \multicolumn{4}{|l|}{ Medication use history (\%) } \\
\hline ACE inhibitor* & 11.8 & 11.6 & 0.005 \\
\hline Anticoagulant* & 1.9 & 1.8 & 0.005 \\
\hline Angiotensin receptor blocker* & 6.8 & 6.8 & 0.002 \\
\hline Aspirin & 0.4 & 0.4 & 0.001 \\
\hline $\mathrm{Na}^{+}$-channel blocker* & 0.2 & 0.2 & 0.002 \\
\hline Beta-adrenergic blocker* & 10.2 & 10.1 & 0.003 \\
\hline K+-channel blocker* & 0.3 & 0.3 & 0.002 \\
\hline $\mathrm{Ca}^{2+}$-channel blocker* & 6.8 & 6.7 & 0.000 \\
\hline Digoxin & 0.3 & 0.3 & 0.002 \\
\hline Loop diuretic* & 2.1 & 2.2 & 0.003 \\
\hline Other diuretic* & 12.9 & 12.8 & 0.004 \\
\hline Insulin* & 2.1 & 2.1 & 0.001 \\
\hline Oral antidiabetic & 7.1 & 7.1 & 0.001 \\
\hline Statin* & 17.7 & 17.6 & 0.003 \\
\hline Fibrate lipid-lowering agent* & 2.2 & 2.2 & 0.000 \\
\hline Nitrate anti-anginal & 0.9 & 0.9 & 0.002 \\
\hline Other antihypertensive* & 2.4 & 2.5 & 0.007 \\
\hline Peripheral vasodilator* & 0.2 & 0.2 & 0.001 \\
\hline Platelet inhibitor, not aspirin* & 1.2 & 1.2 & 0.000 \\
\hline Tricyclic/tetracyclic antidepressant* & 2.6 & 2.6 & 0.002 \\
\hline SSRI/SNRI antidepressant* & 12.9 & 12.8 & 0.004 \\
\hline Trazodone antidepressant* & 2.1 & 2.1 & 0.001 \\
\hline Other antidepressant* & 3.4 & 3.4 & 0.000 \\
\hline Lithium antipsychotic* & 0.2 & 0.2 & 0.001 \\
\hline Atypical antipsychotics* & 1.3 & 1.3 & 0.001 \\
\hline
\end{tabular}




\begin{tabular}{|l|c|c|c|}
\hline Other antipsychotics* & 0.4 & 0.4 & 0.002 \\
\hline Benzodiazepine anticonvulsants* & 7.3 & 7.2 & 0.004 \\
\hline GABA agonist anticonvulsant* & 3.5 & 3.5 & 0.002 \\
\hline Hydroxyzine* & 0.9 & 0.9 & 0.000 \\
\hline Adrenergic beta-agonist* & 10.4 & 10.5 & 0.002 \\
\hline Other bronchodilator* & 1.0 & 1.0 & 0.003 \\
\hline Healthcare Utilization (\%) & & & \\
\hline $\begin{array}{l}\text { Any prescription (other than study ABX) past 30 } \\
\text { days* }\end{array}$ & 67.9 & 66.2 & 0.036 \\
\hline Any antibiotic use past 30 days* & 8.6 & 8.3 & 0.008 \\
\hline Any outpatient visit past 30 days* & 45.0 & 45.1 & 0.002 \\
\hline Any outpatient visits 30-365 days* & 90.4 & 90.2 & 0.004 \\
\hline Any ED visit past 7 days & 1.1 & 1.1 & 0.001 \\
\hline Any hospitalization past 30 days & 0.4 & 0.4 & 0.003 \\
\hline Any hospitalization past 30-365 days* & 8.6 & 8.5 & 0.000 \\
\hline
\end{tabular}

* Covariates included the propensity score model

C-statistic: 0.602

Hosmer and Lemeshow Goodness-of-Fit (GOF) test: $\chi^{2}=167.4(\mathrm{df}=8$, p-value $<0.0001)$ $\mathrm{ABX}=$ antibiotics, $\mathrm{ACE}=$ angiotensin converting enzyme, $\mathrm{AMO}=$ amoxicillin, $\mathrm{COPD}=$ chronic obstructive pulmonary disease, $\mathrm{ED}=$ emergency department, $\mathrm{FLQ}=$ fluoroquinolone antibiotics, GABA = gamma-amino butyric acid 
Appendix F. Sensitivity analysis

Appendix Figure F.1 Cumulative incidence of ventricular arrhythmia and sudden death with azithromycin and amoxicillin in all diagnoses

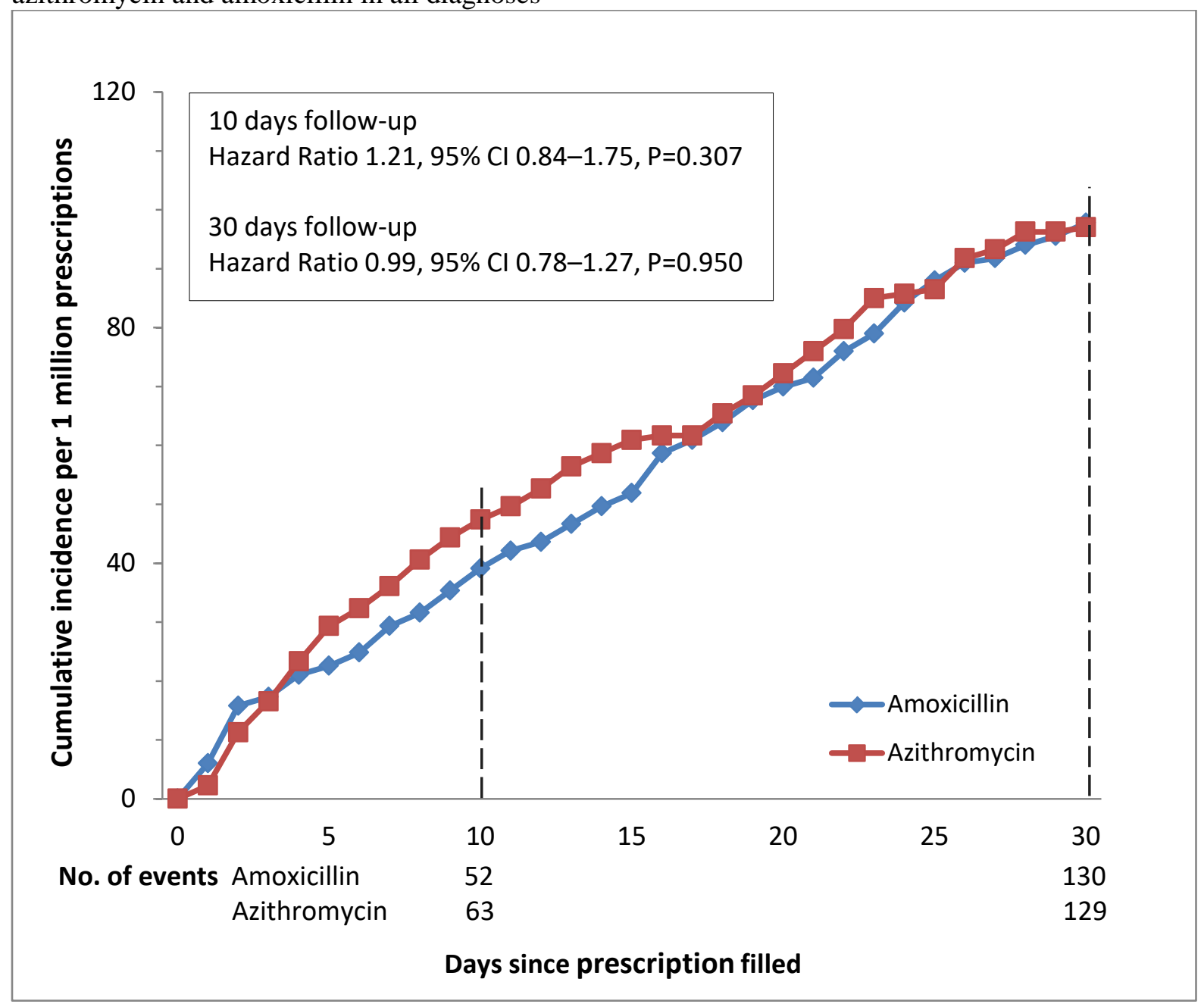


Appendix Figure F.2 Cumulative incidence of ventricular arrhythmia and sudden death with fluoroquinolones and amoxicillin in all diagnoses

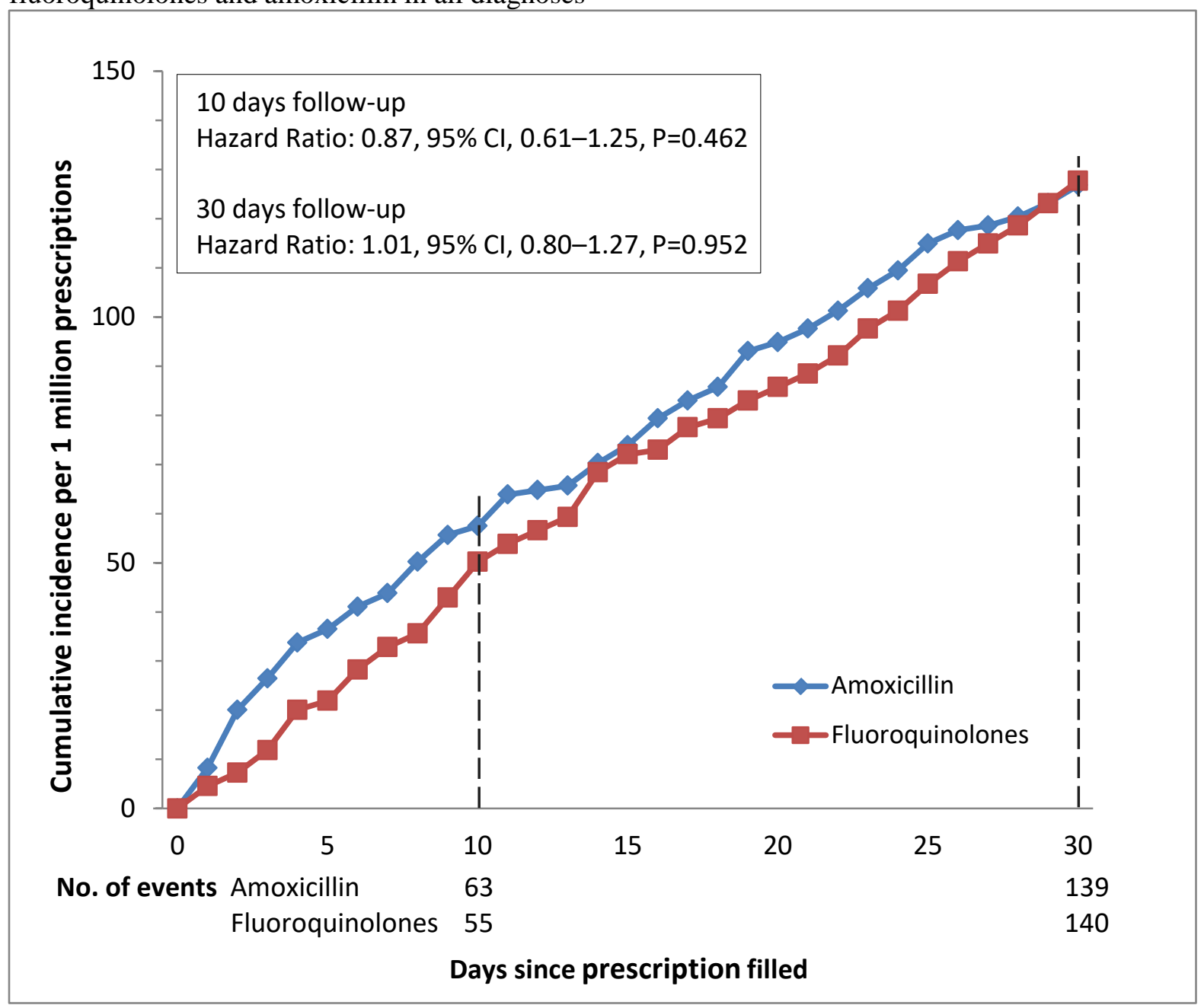




\section{BIBLIOGRAPHY}

Aerssens J, Paulussen AD. Pharmacogenomics and acquired long QT syndrome. Pharmacogenomics. 2005;6(3):259-270.

Albert RK, Schuller JL, Network CCR. Macrolide antibiotics and the risk of cardiac arrhythmias. Am J Respir Crit Care Med. 2014;189(10):1173-1180.

Armahizer MJ, Seybert AL, Smithburger PL, Kane-Gill SL. Drug-drug interactions contributing to QT prolongation in cardiac intensive care units. Journal of critical care. 2013;28(3):243-249.

Astrom-Lilja C, Odeberg JM, Ekman E, Hagg S. Drug-induced torsades de pointes: a review of the Swedish pharmacovigilance database. Pharmacoepidemiology and drug safety. 2008;17(6):587-592.

Austin PC. A Tutorial and Case Study in Propensity Score Analysis: An Application to Estimating the Effect of In-Hospital Smoking Cessation Counseling on Mortality. Multivariate Behav Res. 2011;46(1):119-151.

Austin PC. An Introduction to Propensity Score Methods for Reducing the Effects of Confounding in Observational Studies. Multivariate Behav Res. 2011;46(3):399-424.

Austin PC. The use of propensity score methods with survival or time-to-event outcomes: reporting measures of effect similar to those used in randomized experiments. Stat Med. 2014;33(7):1242-1258.

Briasoulis A, Agarwal V, Pierce WJ. QT prolongation and torsade de pointes induced by fluoroquinolones: infrequent side effects from commonly used medications. Cardiology. 2011;120(2):103-110.

Charlson M, Szatrowski TP, Peterson J, Gold J. Validation of a combined comorbidity index. Journal of clinical epidemiology. 1994;47(11):1245-1251.

Chen HL, Hsiao FY. Domperidone, cytochrome P450 3A4 isoenzyme inhibitors and ventricular arrhythmia: a nationwide case-crossover study. Pharmacoepidemiology and drug safety. 2015;24(8):841-848.

Chiang CE. Congenital and acquired long QT syndrome. Current concepts and management. Cardiology in review. 2004;12(4):222-234. 
Chou HW, Wang JL, Chang CH, Lai CL, Lai MS, Chan KA. Risks of cardiac arrhythmia and mortality among patients using new-generation macrolides, fluoroquinolones, and beta-lactam/beta-lactamase inhibitors: a Taiwanese nationwide study. Clinical infectious diseases : an official publication of the Infectious Diseases Society of America. 2015;60(4):566-577.

Chung CP, Murray KT, Stein CM, Hall K, Ray WA. A computer case definition for sudden cardiac death. Pharmacoepidemiology and drug safety.

2010;19(6):563-572.

Ciprofloxacin [package insert]. Wayne, NJ: Bayer HealthCare Pharmaceuticals Inc; 2013. https://www.accessdata.fda.gov/drugsatfda_docs/label/2013/019857s062lbl.pd f, April 17, 2017.

Cocco G, Jerie P. Torsades de pointes induced by the concomitant use of ivabradine and azithromycin: an unexpected dangerous interaction. Cardiovasc Toxicol. 2015;15(1):104-106.

De Bruin ML, van Hemel NM, Leufkens HG, Hoes AW. Hospital discharge diagnoses of ventricular arrhythmias and cardiac arrest were useful for epidemiologic research. Journal of clinical epidemiology. 2005;58(12):1325-1329.

Denny JC, Miller RA, Waitman LR, Arrieta MA, Peterson JF. Identifying QT prolongation from ECG impressions using a general-purpose Natural Language Processor. Int J Med Inform. 2009;78 Suppl 1:S34-42.

Dresser GK, Spence JD, Bailey DG. Pharmacokinetic-pharmacodynamic consequences and clinical relevance of cytochrome P450 3A4 inhibition. Clin Pharmacokinet. 2000;38(1):41-57.

Enger C, Cali C, Walker AM. Serious ventricular arrhythmias among users of cisapride and other QT-prolonging agents in the United States. Pharmacoepidemiology and drug safety. 2002;11(6):477-486.

Eppler S, Gordon MS, Redfern CH, et al. Lack of a pharmacokinetic interaction between trastuzumab and carboplatin in the presence of docetaxel: results from a phase Ib study in patients with HER2-positive metastatic or locally advanced inoperable solid tumors. Anticancer Drugs. 2015;26(4):448-455.

European Medicines Agency. Guideline on good pharmacovigilance practices (GVP) Module VIII - Post-authorisation safety studies (Rev 2) 2016; http://www.ema.europa.eu/docs/en_GB/document_library/Scientific_guideline /2012/06/WC500129137.pdf. Accessed September 20, 2016. 
Finlayson K, Witchel HJ, McCulloch J, Sharkey J. Acquired QT interval prolongation and HERG: implications for drug discovery and development. European journal of pharmacology. 2004;500(1-3):129-142.

Fishman GI, Chugh SS, Dimarco JP, et al. Sudden death prediction and prevention: report from a National Heart, Lung, and Blood Institute and Heart Rhythm Society Workshop. Circulation. 2010;122(22):2335-2348.

Goldberger JJ, Buxton AE, Cain M, et al. Risk stratification for arrhythmic sudden cardiac death: identifying the roadblocks. Circulation. 2011;123(21):24232430.

Hancox JC, Hasnain M, Vieweg WV, Crouse EL, Baranchuk A. Azithromycin, cardiovascular risks, QTc interval prolongation, torsade de pointes, and regulatory issues: A narrative review based on the study of case reports. Ther Adv Infect Dis. 2013;1(5):155-165.

Hancox JC, Hasnain M, Vieweg WV, Gysel M, Methot M, Baranchuk A. Erythromycin, QTc interval prolongation, and torsade de pointes: Case reports, major risk factors and illness severity. Ther Adv Infect Dis. 2014;2(2):47-59.

Hancox JC, McPate MJ, El Harchi A, Zhang YH. The hERG potassium channel and hERG screening for drug-induced torsades de pointes. Pharmacol Ther. 2008;119(2):118-132.

Hanrahan JP, Choo PW, Carlson W, Greineder D, Faich GA, Platt R. Terfenadineassociated ventricular arrhythmias and QTc interval prolongation. A retrospective cohort comparison with other antihistamines among members of a health maintenance organization. Annals of epidemiology. 1995;5(3):201209.

Hennessy S, Leonard CE, Freeman CP, et al. Validation of diagnostic codes for outpatient-originating sudden cardiac death and ventricular arrhythmia in Medicaid and Medicare claims data. Pharmacoepidemiology and drug safety. 2010;19(6):555-562.

Hennessy S, Leonard CE, Newcomb C, Kimmel SE, Bilker WB. Cisapride and ventricular arrhythmia. British journal of clinical pharmacology. 2008;66(3):375-385.

Hoffler D, Koeppe P, Paeske B. Pharmacokinetics of azithromycin in normal and impaired renal function. Infection. 1995;23(6):356-361. 
Hosmer D.W LS, Sturdivant RX. Applied Logistic Regression. 3rd ed.

Inghammar M, Svanstrom H, Melbye M, Pasternak B, Hviid A. Oral fluoroquinolone use and serious arrhythmia: bi-national cohort study. BMJ. 2016;352:1843.

Isbister GK, Page CB. Drug induced QT prolongation: the measurement and assessment of the QT interval in clinical practice. British journal of clinical pharmacology. 2013;76(1):48-57.

Isbister GK. Risk assessment of drug-induced QT prolongation. Aust Prescr. 2015;38(1):20-24.

Johannes CB, Varas-Lorenzo C, McQuay LJ, Midkiff KD, Fife D. Risk of serious ventricular arrhythmia and sudden death in a cohort of users of domperidone: a nested case-control study. Pharmacoepidemiology and drug safety. 2010;19(9):881-888.

Josephson M, Wellens HJ. Implantable defibrillators and sudden cardiac death. Circulation. 2004;109(22):2685-2691.

Kannankeril P, Roden DM, Darbar D. Drug-induced long QT syndrome. Pharmacol Rev. 2010;62(4):760-781.

Kawai VK, Murray KT, Stein CM, et al. Validation of a computer case definition for sudden death in opioid users. BMC Res Notes. 2012;5:473.

Kestenbaum B, Rudser KD, Shlipak MG, et al. Kidney function, electrocardiographic findings, and cardiovascular events among older adults. Clin J Am Soc Nephrol. 2007;2(3):501-508.

Kim MH, Berkowitz C, Trohman RG. Polymorphic ventricular tachycardia with a normal QT interval following azithromycin. Pacing Clin Electrophysiol. 2005;28(11):1221-1222.

Kleinbaum DG KM. Survival Analysis A Self-Learning Text. 3rd ed.

Leonard CE, Bilker WB, Newcomb C, Kimmel SE, Hennessy S. Antidepressants and the risk of sudden death and ventricular arrhythmia. Pharmacoepidemiology and drug safety. 2011;20(9):903-913.

Leonard CE, Freeman CP, Newcomb CW, et al. Antipsychotics and the Risks of Sudden death and All-Cause Death: Cohort Studies in Medicaid and Dually- 
Eligible Medicaid-Medicare Beneficiaries of Five States. Journal of clinical \& experimental cardiology. 2013;Suppl 10(6):1-9.

Liu HH. Safety profile of the fluoroquinolones: focus on levofloxacin. Drug Saf. 2010;33(5):353-369.

Ma TK, Chow KM, Choy AS, Kwan BC, Szeto CC, Li PK. Clinical manifestation of macrolide antibiotic toxicity in CKD and dialysis patients. Clin Kidney J. 2014;7(6):507-512.

Mayo Foundation for Medical Education and Research MC. Long QT syndrome. 2015; http://www.mayoclinic.org/diseases-conditions/long-qtsyndrome/basics/symptoms/con-20025388. Accessed February 24, 2016.

McDonald KM, Hlatky MA, Saynina O, Geppert J, Garber AM, McClellan MB. Trends in hospital treatment of ventricular arrhythmias among Medicare beneficiaries, 1985 to 1995. American heart journal. 2002;144(3):413-421.

Mehrzad R, Barza M. Weighing the adverse cardiac effects of fluoroquinolones: A risk perspective. J Clin Pharmacol. 2015;55(11):1198-1206.

Molokhia M, Pathak A, Lapeyre-Mestre M, et al. Case ascertainment and estimated incidence of drug-induced long-QT syndrome: study in Southwest France. British journal of clinical pharmacology. 2008;66(3):386-395.

Mortensen EM, Halm EA, Pugh MJ, et al. Association of azithromycin with mortality and cardiovascular events among older patients hospitalized with pneumonia. JAMA. 2014;311(21):2199-2208.

Nachimuthu S, Assar MD, Schussler JM. Drug-induced QT interval prolongation: mechanisms and clinical management. Ther Adv Drug Saf. 2012;3(5):241-253.

Niedrig D, Maechler S, Hoppe L, Corti N, Kovari H, Russmann S. Drug safety of macrolide and quinolone antibiotics in a tertiary care hospital: administration of interacting co-medication and QT prolongation. Eur J Clin Pharmacol. 2016;72(7):859-867.

Owens RC, Jr., Nolin TD. Antimicrobial-associated QT interval prolongation: pointes of interest. Clinical infectious diseases : an official publication of the Infectious Diseases Society of America. 2006;43(12):1603-1611.

Patane S. Torsade de pointes, QT interval prolongation and renal disease. Int J Cardiol. 2011;149(2):241-242. 
Paulussen AD, Aerssens J. Risk factors for drug-induced long-QT syndrome. Neth Heart J. 2005;13(2):47-56.

Pearson EC, Woosley RL. QT prolongation and torsades de pointes among methadone users: reports to the FDA spontaneous reporting system. Pharmacoepidemiology and drug safety. 2005;14(11):747-753.

Quan H, Li B, Couris CM, et al. Updating and validating the Charlson comorbidity index and score for risk adjustment in hospital discharge abstracts using data from 6 countries. Am J Epidemiol. 2011;173(6):676-682.

Rao GA, Mann JR, Shoaibi A, et al. Azithromycin and levofloxacin use and increased risk of cardiac arrhythmia and death. Annals of family medicine. 2014;12(2):121-127.

Ray WA, Murray KT, Hall K, Arbogast PG, Stein CM. Azithromycin and the risk of cardiovascular death. N Engl J Med. 2012;366(20):1881-1890.

Ray WA, Murray KT, Meredith S, Narasimhulu SS, Hall K, Stein CM. Oral erythromycin and the risk of sudden death from cardiac causes. N Engl J Med. 2004;351(11):1089-1096.

Roden DM. Clinical practice. Long-QT syndrome. N Engl J Med. 2008;358(2):169176.

Roden DM. Drug-induced prolongation of the QT interval. N Engl J Med. 2004;350(10):1013-1022.

Roe CM, Odell KW, Henderson RR. Concomitant use of antipsychotics and drugs that may prolong the QT interval. J Clin Psychopharmacol. 2003;23(2):197-200.

Rosenbaum PR, Rubin DB. Reducing bias in observational studies using subclassification on the propensity score. Journal of the American Statistical Association. 1984;79(387):516-524.

Schelleman H, Bilker WB, Kimmel SE, et al. Methylphenidate and risk of serious cardiovascular events in adults. Am J Psychiatry. 2012;169(2):178-185.

Strom BL KS. Textbook of Pharmacoepidemiology. 1st ed.

Svanstrom H, Pasternak B, Hviid A. Use of azithromycin and death from cardiovascular causes. N Engl J Med. 2013;368(18):1704-1712. 
Tamariz L, Harkins T, Nair V. A systematic review of validated methods for identifying ventricular arrhythmias using administrative and claims data. Pharmacoepidemiology and drug safety. 2012;21 Suppl 1:148-153.

Tobi H, Faber A, van den Berg PB, Drane JW, de Jong-van den Berg LT. Studying comedication patterns: the impact of definitions. Pharmacoepidemiology and drug safety. 2007;16(4):405-411.

Trac MH, McArthur E, Jandoc R, et al. Macrolide antibiotics and the risk of ventricular arrhythmia in older adults. CMAJ. 2016;188(7):E120-129.

Trojak B, Astruc K, Pinoit JM, et al. Hypokalemia is associated with lengthening of QT interval in psychiatric patients on admission. Psychiatry Res. 2009;169(3):257-260.

U.S. Department of Health and Human Services NIoH, National Heart, Lung and Blood Institute. What Are the Signs and Symptoms of Long QT Syndrome? 2011; https://www.nhlbi.nih.gov/health/health-topics/topics/qt/signs\#. Accessed February 24, 2016.

U.S. Department of Health and Human Services USFDA. FDA Drug Safety Communication: Azithromycin (Zithromax or Zmax) and the risk of potentially fatal heart rhythms. 2013; http://www.fda.gov/Drugs/DrugSafety/ucm341822.htm. Accessed September 20, 2016.

van Noord C, Eijgelsheim M, Stricker BH. Drug- and non-drug-associated QT interval prolongation. British journal of clinical pharmacology. 2010;70(1):16-23.

von Rosensteil NA, Adam D. Macrolide antibacterials. Drug interactions of clinical significance. Drug Saf. 1995;13(2):105-122.

Watkins PB. Drug metabolism by cytochromes P450 in the liver and small bowel. Gastroenterol Clin North Am. 1992;21(3):511-526.

Woosley RL, Romero KA. QTdrugs List. www.Crediblemeds.org. Accessed March 8, 2017.

Wu CS, Tsai YT, Tsai HJ. Antipsychotic drugs and the risk of ventricular arrhythmia and/or sudden cardiac death: a nation-wide case-crossover study. J Am Heart Assoc. 2015;4(2). 
Yap YG, Camm AJ. Drug induced QT prolongation and torsades de pointes. Heart. 2003;89(11):1363-1372.

Zambon A, Polo Friz H, Contiero P, Corrao G. Effect of macrolide and fluoroquinolone antibacterials on the risk of ventricular arrhythmia and cardiac arrest: an observational study in Italy using case-control, case-crossover and case-time-control designs. Drug Saf. 2009;32(2):159-167.

Zanolari Calderari M, Vigier RO, Bettinelli A, Bianchetti MG. Electrocardiographic QT prolongation and sudden death in renal hypokalemic alkalosis. Nephron. 2002;91(4):762-763.

ZITHROMAX(R) [package insert]. New York, NY: Pfizer Pharmaceuticals Inc; 2017. http://labeling.pfizer.com/ShowLabeling.aspx?id=511, April 17, 2017. 